\title{
COUNTEREXAMPLES FOR OPTIMAL SCALING OF METROPOLIS-HASTINGS CHAINS WITH ROUGH TARGET DENSITIES
}

\author{
BY JURE VOGRINC** AND WILFRID S. KENDALL ${ }^{\dagger}$ \\ Department of Statistics, University of Warwick, ${ }^{*}$ jure.vogrinc@warwick.ac.uk; ${ }^{\dagger}$ w.s.kendall@warwick.ac.uk
}

\begin{abstract}
For sufficiently smooth targets of product form it is known that the variance of a single coordinate of the proposal in RWM (random walk Metropolis) and MALA (Metropolis adjusted Langevin algorithm) should optimally scale as $n^{-1}$ and as $n^{-\frac{1}{3}}$ with dimension $n$, and that the acceptance rates should be tuned to 0.234 and 0.574 . We establish counterexamples to demonstrate that smoothness assumptions of the order of $\mathcal{C}^{1}(\mathbb{R})$ for RWM and $\mathcal{C}^{3}(\mathbb{R})$ for MALA are indeed required if these scaling rates are to hold. The counterexamples identify classes of marginal targets for which these guidelines are violated, obtained by perturbing a standard normal density (at the level of the potential for RWM and the second derivative of the potential for MALA) using roughness generated by a path of fractional Brownian motion with Hurst exponent $H$. For such targets there is strong evidence that RWM and MALA proposal variances should optimally be scaled as $n^{-\frac{1}{H}}$ and as $n^{-\frac{1}{2+H}}$ and will then obey anomalous acceptance rate guidelines. Useful heuristics resulting from this theory are discussed. The paper develops a framework capable of tackling optimal scaling results for quite general Metropolis-Hastings algorithms (possibly depending on a random environment).
\end{abstract}

1. Introduction. Probabilistic computation and optimisation are tools of widespread importance in applied mathematical science, and are widely used in order to facilitate the use of Bayesian statistics, especially in machine learning contexts. In particular, the use of Markov chain Monte Carlo (MCMC) methods is now wide-spread. This greatly increases the value of mathematical theory underlying these methods; many significant theoretical advances have indeed been made but theory still lags behind the explosive growth of many varieties of applications. Theory typically provides significant help and guidance by studying basic building blocks of these algorithms, applied to toy examples which are nevertheless representative of applications [5, 31].

A remarkable example of theoretical guidance is provided by results on optimal scaling of MCMC (Roberts, Gelman and Gilks [29], Roberts and Rosenthal [30], see also Gelfand and Mitter [8] who establish diffusion limits for MCMC algorithms). Here the toy examples are high-dimensional "product targets" (multivariate probability densities which render all $n$ coordinates independent and identically distributed). Optimal scaling results show that (under suitable regularity conditions) as dimension $n$ increases so the proposal variances of each coordinate of the random walk Metropolis (RWM) and the Metropolis adjusted Langevin algorithm (MALA) proposals should respectively be chosen proportional to $n^{-1}$ and $n^{-1 / 3}$. Furthermore, it proves optimal as $n \rightarrow \infty$ to choose the constant of proportionality so as to obtain average acceptance rates of 0.234 of the proposed moves for RWM and 0.574 for MALA. These results were originally proved only for the toy example of product targets

Received October 2019; revised July 2020.

MSC2020 subject classifications. Primary 60J22; secondary 65C05, $60 \mathrm{~F} 05$.

Key words and phrases. Anomalous optimal scaling, expected square jump distance, fractional Brownian motion, Markov chain Monte Carlo, Metropolis-adjusted Langevin algorithm, Metropolis-Hastings, random walk Metropolis. 
given above; nevertheless simulation evidence suggests that they should hold in much greater generality and notable progress has been made to generalise the theory towards more general targets, especially in the case of RWM: see, for example, Yang, Roberts and Rosenthal [34]. Consequently the theory does indeed provide very practical and useful guidelines for practitioners, and additionally provides an important context for motivating and assessing adaptive MCMC methods.

The theoretical results require smoothness assumptions for the underlying marginal target density function. Roberts, Gelman and Gilks [29] actually required three continuous derivatives for their RWM result while Roberts and Rosenthal [30] needed 8 continuous derivatives for their approach to MALA. These assumptions were necessitated by the methods of proof but did not otherwise seem particularly natural and it was unclear to what extent they were actually necessary. Recent work has used different methods of proof to establish, at least in the case of RWM, that the original smoothness assumptions were indeed much stricter than is really required $[7,35]$. The main focus of our paper is to develop a class of counterexamples to demonstrate the extent to which some kinds of smoothness assumption are genuinely necessary for both RWM and MALA.

In summary, we show that a certain level of smoothness of the marginal target density function is indeed required in order to deliver the original optimal scaling guidelines. To be specific, RWM essentially requires 1 continuous derivative almost everywhere while MALA requires three continuous derivatives almost everywhere. Note that no derivatives are required in order for RWM to deliver the prescribed target probability measure as a large-time equilibrium, while MALA requires just one derivative. Nevertheless we show that some higher-order smoothness is indeed necessary if the algorithms are to scale in a standard way. In the following it is shown that, in the absence of suitable smoothness, there exist classes of targets for which the above optimality results do not apply, and indeed different, anomalous, tuning guidelines appear to be optimal. Note in particular that failure of smoothness at isolated points (as often occurs in applications) need not be sufficient to destroy standard smoothing [7]: our counterexamples are necessarily nonsmooth over a substantial range. However, the counterexamples tell us something fundamental about the way in which RWM and MALA really do depend on regularity and are thus methodologically interesting. They quantifiably exhibit another, often overlooked, way in which MCMC can perform badly, different for instance from the target having multiple modes or being zero in large parts of space. A bottleneck in MCMC mixing can also be caused by local roughness or oscillations and we believe the results presented below do indicate useful aspects of scaling behaviour for MCMC methods in such cases (see Section 8.3).

For RWM, for each $0<H<1$ we use a probabilistic approach to construct a class of product targets which lie in $\mathcal{C}^{\gamma}(\mathbb{R})$ (for $\gamma<H$ ) but not in $\mathcal{C}^{H}(\mathbb{R}$ ), and for which the RWM algorithm does not scale optimally in the way indicated by the theory in [29]. Indeed an "expected squared jump distance" (ESJD) approach indicates a different and anomalous manner of optimal scaling. For MALA, for each $0<H<1$ we similarly use a probabilistic approach to construct a class of product targets which lie in $\mathcal{C}^{2+\gamma}(\mathbb{R})\left(\right.$ for $\gamma<H$ ) but not in $\mathcal{C}^{2+H}(\mathbb{R})$ and for which again the MALA algorithm does not scale optimally according to the regularcase theory of Roberts and Rosenthal [30]; here an ESJD approach again indicates a different and anomalous manner of optimal scaling.

Our method of approach is to generate random targets - in effect, random environmentsbased on a random realisation of a two-sided $H$-fractional Brownian motion path. Indeed, bearing in mind appropriate density theorems for Gaussian measures, in some sense our counterexamples are generic! We use the generated path to construct a marginal probability density function such that any possibility of optimal scaling could only arise by tuning the coordinate variance of proposals for the associated $n$-dimensional product targets to be 
proportional to $n^{-1 / H}$ for RWM and to $n^{-1 /(2+H)}$ for MALA (instead of $n^{-1}$ for RWM and $n^{-1 / 3}$ for MALA).

In addition the method of proof may be of independent interest. Section 3 provides a very suitable framework for addressing optimal scaling questions for Metropolis-class MCMC methods, particularly for identifying minimal required smoothness conditions. It is plausible that similar frameworks can be obtained for other classes of MCMC algorithms. Independently of that, an approach involving random targets, similar to Section 5, could be used to construct other kinds of counterexamples in MCMC.

The rest of the paper is organised as follows. Section 2 states and discusses the main results of the paper. Section 3 establishes conditions, nearly as general as possible, in the setting of product targets under which an associated central limit theorem holds for the log MetropolisHastings ratio and a non-trivial limiting acceptance rate exists. Section 4 states and proves consequences of the celebrated Isserlis theorem which will later be used to control distributions of important quantities expressed in the context of a random environment. Section 5 introduces a general framework for showing when anomalous scaling can occur for general Metropolis-Hastings algorithms applied to product targets in which the marginal product target density depends on a random environment which is a continuous Gaussian process. Section 6 and Section 7 respectively verify that the general framework of Section 5 is satisfied in cases of anomalously scaled RWM and MALA. Targets used for RWM (respectively MALA) are perturbations, on the level of potential (respectively second derivative of the potential), of the standard normal density. Finally, Section 8 discusses considerations concerning expected square jump distance, open questions, potential extensions and how heuristics suggested by these theoretical results could be useful in applications.

2. Main results of the paper. This section presents our main results in more detail. First of all, recall the mathematical framework of optimal scaling for MCMC. The marginal probability density function for the product target measure (assumed here to be strictly positive) is denoted by $\pi$. Thus the product target measure on $\mathbb{R}^{n}$ is given by

$$
\Pi_{n}(\mathrm{~d} x)=\Pi_{n}\left(\mathrm{~d} x_{1}, \ldots, \mathrm{d} x_{n}\right)=\prod_{i=1}^{n}\left(\pi\left(x_{i}\right) \mathrm{d} x_{i}\right) .
$$

Our results concern asymptotic behaviour (as the dimension $n$ grows to $\infty$ ) of MCMC algorithms which deliver this target measure as large-time equilibrium. The relevant algorithms, RWM and MALA, give rise to Markov chains $\left(X_{k}^{\mathrm{RWM},(n)}: k=1,2, \ldots\right)$ for RWM and $\left(X_{k}^{\mathrm{MALA},(n)}: k=1,2, \ldots\right)$ for MALA (here the dummy index $k$ is the discrete time variable for the Markov chains). The chains are Metropolis-Hastings (MH) algorithms with target probability measures $\Pi_{n}$ based on multivariate normal proposals $Q^{\mathrm{RWM},(n)}(x, \mathrm{~d} y) \sim$ $N\left(x, \frac{\ell^{2}}{n} \cdot I_{n}\right)$ for RWM and $Q^{\operatorname{MALA},(n)}(x, \mathrm{~d} y) \sim N\left(x+\frac{\ell^{2}}{2 n^{1 / 3}} \nabla\left(\log \Pi_{n}(x)\right), \frac{\ell^{2}}{n^{1 / 3}} \cdot I_{n}\right)$ for MALA, and we consider the stationary versions of all these chains (so initial distribution is always $\Pi_{n}$ ). Here $\ell>0$ is a parameter determining the asymptotic scale of the proposal.

The classic results of Roberts, Gelman and Gilks [29] and [30] state (respectively for $\pi \in \mathcal{C}^{3}(\mathbb{R})$ for RWM, and $\pi \in \mathcal{C}^{8}(\mathbb{R})$ for MALA) that as $n \rightarrow \infty$ there is weak convergence of the first coordinate of the chain (under certain conditions on the decay of the tails and the regularity of the marginal probability density of $\Pi_{n}$ )

$$
X_{\lfloor n \cdot t\rfloor, 1}^{\mathrm{RWM},(n)} \stackrel{w}{\rightarrow} U_{t} \quad \text { and } \quad X_{\left\lfloor n^{1 / 3} \cdot t\right\rfloor, 1}^{\mathrm{MALA},(n)} \stackrel{w}{\rightarrow} U_{t}
$$

to a Langevin diffusion $U$, a solution of the continuous time stochastic differential equation

$$
\mathrm{d} U_{t}=h(\ell)^{1 / 2} \mathrm{~d} B_{t}+\frac{h(\ell)}{2} \nabla\left(\log \left(\pi\left(U_{t}\right)\right) \mathrm{d} t .\right.
$$


That is to say, the accelerated first coordinates $X_{\lfloor n t\rfloor, 1}^{\mathrm{RWM},(n)}$ and $X_{\left\lfloor n^{1 / 3} t\right\rfloor, 1}^{\mathrm{MALA},(n)}$, when considered as piece-wise constant continuous time processes, converge weakly to the Langevin diffusion $U$ as the dimension $n$ increases. The expressions for diffusion speeds $h(\ell)$ are different in RWM and MALA cases and optimising over the choice of $\ell$ then leads to different (but appealingly simple) acceptance ratio guidelines.

The computational heart of these results lies in the task of showing that the acceptance ratio converges to a constant different to zero or one, and this follows by application of a version of the central limit theorem (CLT) that applies to the coordinate-wise logarithms of $\mathrm{MH}$ acceptance ratios for these algorithms. For instance, if $X_{1}, \ldots, X_{n}$ are the independent and identically distributed (IID) coordinates of $X^{\mathrm{RWM},(n)} \sim \Pi_{n}$ and $Y_{1}, \ldots, Y_{n}$ are the IID coordinates of the RWM proposal $Y^{\mathrm{RWM},(n)} \sim Q^{\mathrm{RWM},(n)}\left(X^{\mathrm{RWM},(n)}, \mathrm{d} y\right)$ then the following CLT

$$
\sum_{i=1}^{n} \log \left(\pi\left(Y_{i}\right)\right)-\log \left(\pi\left(X_{i}\right)\right) \stackrel{w}{\rightarrow} N\left(-\frac{1}{2} \sigma^{2}, \sigma^{2}\right)
$$

holds for an appropriate constant $\left.\sigma^{2}=\ell^{2} \int_{\mathbb{R}}\left((\log \pi)^{\prime}(x)\right)\right)^{2} \pi(x) \mathrm{d} x$. This then identifies the limiting average acceptance ratio via

$$
\begin{aligned}
\alpha\left(X^{\mathrm{RWM},(n)}, Y^{\mathrm{RWM},(n)}\right) & =(1 \wedge \exp )\left(\sum_{i=1}^{n} \log \left(\pi\left(Y_{i}\right)\right)-\log \left(\pi\left(X_{i}\right)\right)\right) \\
& \stackrel{w}{\rightarrow}(1 \wedge \exp )\left(N\left(-\frac{1}{2} \sigma^{2}, \sigma^{2}\right)\right),
\end{aligned}
$$

where $(1 \wedge \exp )(x)$ denotes $\min \left(1, e^{x}\right)$ for $x \in \mathbb{R}$. Note that this identifies the optimal scaling rate for the coordinates of the proposal: if a scaling rate is not asymptotic to the rate giving a CLT $\left(n^{-1}\right.$ for RWM and $n^{-1 / 3}$ for MALA) then either there is no limiting average acceptance rate or the limit is necessarily 0 or 1 .

We now construct classes of marginal probability density functions for which anomalous scaling occurs at least at the level of ESJD. We do this by using a randomised construction based on fractional Brownian motions. Recall that $\left\{B_{x}^{(H)}, x \in \mathbb{R}\right\}$ is a two-sided fractional Brownian motion (fBM) with Hurst parameter $H \in(0,1)$ if it is a centred zero-mean Gaussian process with covariance defined for arbitrary $x, y \in \mathbb{R}$ by

$$
\Gamma^{(H)}(x, y)=\mathbb{E}\left[B_{x}^{(H)} B_{y}^{(H)}\right]=\frac{1}{2}|x|^{2 H}+\frac{1}{2}|y|^{2 H}-\frac{1}{2}|x-y|^{2 H} .
$$

Here we refer to Nualart [24], Chapter 5, for fBM theory. This reference covers the singlesided fBM with $x \geq 0$ : however extension to the double-sided case is immediate if one notes that (2) remains nonnegative definite for all $x, y$. Revuz and Yor [28], Chapter I, Exercise (3.9), gives an explicit and succinct construction for all $x, y$ (see also [21]). The sample paths of fBM with Hurst parameter $H$ are almost surely Hölder continuous of exponent $\gamma$ whenever $0<\gamma<H$ (though not for $\gamma=H$ ). Let $\Omega^{(H)}$ denote the space of all two-sided paths that are zero at time zero and are in $\mathcal{C}^{\gamma}(\mathbb{R})$ for all $0<\gamma<H$, so $\Omega^{(H)}$ is in fact a probability space equipped with a measure provided by two-sided fBM with Hurst parameter $H$.

The main result concerning RWM counterexamples can be summarised as follows (where $I_{n}$ denotes the $n$-dimensional identity matrix).

THEOREM 1 (Anomalous scaling for RWM). Consider the random function $\xi_{B}^{(H)}$ depending on the fractional Brownian motion $B^{(H)}$ and defined by

$$
\xi\left(x \mid B^{(H)}\right)=\frac{1}{\sqrt{2 \pi}} \exp \left(B_{x}^{(H)}-\frac{x^{2}}{2}\right) .
$$


Almost surely $\int_{-\infty}^{\infty} \xi\left(x \mid B^{(H)}\right) d x<\infty$, so $\xi\left(\cdot \mid B^{(H)}\right)$ can be renormalised to provide a (random) target density

$$
\pi\left(x \mid B^{(H)}\right)=\frac{\xi\left(x \mid B^{(H)}\right)}{\int_{-\infty}^{\infty} \xi\left(u \mid B^{(H)}\right) d u} .
$$

Condition on $B^{(H)}$ and consider a stationary $R W M$ chain with target $\Pi_{n}\left(\cdot \mid B^{(H)}\right)=$ $\prod_{i=1}^{n} \pi\left(\cdot \mid B^{(H)}\right)$ and proposal $Q^{\mathrm{RWM},(n)}(x, \mathrm{~d} y) \sim N\left(x, \ell^{2} n^{-1 / H} \cdot I_{n}\right)$. Then there is a constant $\sigma^{2}=\ell^{2 H} \frac{2^{H}}{\sqrt{\pi}} \Gamma\left(H+\frac{1}{2}\right)$ such that, as $n \rightarrow \infty$, the probability of acceptance of the proposal (conditional on the underlying $B^{(H)}$ ) satisfies

$$
\alpha\left(X^{\mathrm{RWM},(n)}, Y^{\mathrm{RWM},(n)}\right) \stackrel{w}{\rightarrow}(1 \wedge \exp )\left(N\left(-\frac{1}{2} \sigma^{2}, \sigma^{2}\right)\right)
$$

almost surely (for almost all realisations of the $\left.f B M B^{(H)}\right)$.

In effect $B^{(H)}$ is providing a random environment, $X^{\mathrm{RWM},(n)}$ is a Markov chain using this random environment, and Theorem 1 refers to the quenched behaviour of this Markov chain in a random environment.

We draw attention to the anomalous rate of scaling of the proposal variances. The reader should keep in mind that substantially different rates of proposal scaling will yield either a trivial limit or no limiting behaviour at all. In particular, the optimal scaling of Roberts, Gelman and Gilks [29] cannot here apply. It is also possible to see that this rate of proposal variance decay is optimal in terms of the ESJD. However, we have to pose it in a slightly different way than classically: for any decay rate of proposal variances the ESJD (random, because it depends on the environment) divided by the optimal ESJD rate converges to zero in probability. We outline the proof and discuss this further in Section 8.1.

Given the Hurst parameter $H$ and the rate $\ell n^{-1 / H}$ of optimal proposal variance decay, one can then optimise the ESJD decay rate over the choice of $\ell$. This gives us an optimal acceptance rate for each $H$. The function cannot be expressed in closed form but can be plotted numerically, see the left panel of Figure 1. Note that the optimal acceptance rate converges to zero as $H \rightarrow 0$ and, for example, it is optimal to accept approximately $7 \%$ of the proposals for $H=1 / 2$ and only $0.7 \%$ for $H=1 / 4$.

The analogous result concerning MALA requires definition of a localisation function $\varphi_{c}(x): \mathbb{R} \rightarrow[0,1]$ depending on a parameter $c>0$ and defined for $x \in \mathbb{R}\left(\right.$ with $\left.\varphi_{c}(0)=1\right)$ as follows

$$
\varphi_{c}(x)=1 \wedge\left(c^{\frac{3}{2 H}}|x|^{-3}\right)=\min \left\{1, c^{\frac{3}{2 H}}|x|^{-3}\right\} .
$$

We will consider perturbations of a normal density by a fBM path at the level of the second derivative of the potential and the localisation function is introduced to control fBM fluctuations and ensure the resulting random target is integrable.

Anomalous scaling of MALA can then occur as follows.

THEOREM 2 (Anomalous scaling for MALA). Consider the random function $\xi(\cdot \mid$ $\left.B^{(H)} ; c\right)$ depending on the fractional Brownian motion $B^{(H)}$ and defined by

$$
\xi\left(x \mid B^{(H)} ; c\right)=\frac{1}{\sqrt{2 \pi}} \exp \left(-\frac{x^{2}}{2}+x^{2} \int_{0}^{1} B_{x s}^{(H)} \varphi_{c}(x s)(1-s) \mathrm{d} s\right) .
$$

For every Hurst index $H \in(0,1)$ there exists a small enough $c>0$, such that almost surely $\int_{-\infty}^{\infty} \xi\left(x \mid B^{(H)} ; c\right) d x$ is finite, so $\xi\left(\cdot \mid B^{(H)} ; c\right)$ can be renormalised to provide a (random) target density

$$
\pi\left(x \mid B^{(H)} ; c\right)=\frac{\xi\left(x \mid B^{(H)} ; c\right)}{\int_{-\infty}^{\infty} \xi\left(u \mid B^{(H)} ; c\right) d u} .
$$



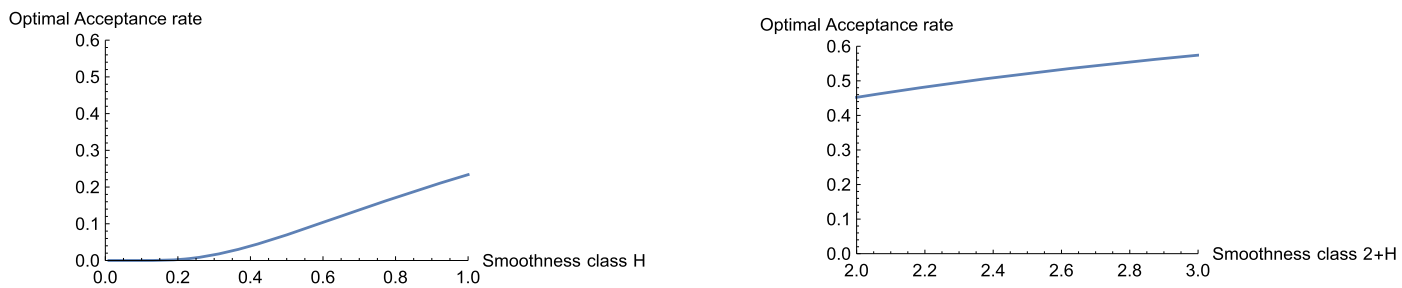

FIG. 1. Optimal acceptance rates for RWM (left) for smoothness classes $H \in(0,1)$ and MALA (right) for smoothness classes $2+H$ with $H \in(0,1)$. The optimal acceptance rates are obtained by numerically solving the same equation (in terms of smoothness class) in both cases.

Condition on $B^{(H)}$ and consider a stationary MALA chain with target $\Pi_{n}\left(\cdot \mid B^{(H)} ; c\right)=$ $\prod_{i=1}^{n} \pi\left(\cdot \mid B^{(H)} ; c\right)$ and proposal $Q^{\mathrm{MALA},(n)}(x, \mathrm{~d} y) \sim N\left(x+\frac{1}{2} \ell^{2} n^{-1 /(2+H)} \nabla\left(\log \Pi_{n}(x)\right)\right.$, $\left.\ell^{2} n^{-1 /(2+H)} \cdot I_{n}\right)$. Then there is a constant $\sigma^{2}>0$ such that, as $n \rightarrow \infty$, the probability of acceptance of the proposal (conditional on the underlying $B^{(H)}$ ) satisfies

$$
\alpha\left(X^{\mathrm{MALA},(n)}, Y^{\mathrm{MALA},(n)}\right) \stackrel{w}{\rightarrow}(1 \wedge \exp )\left(N\left(-\frac{1}{2} \sigma^{2}, \sigma^{2}\right)\right)
$$

almost surely (for almost all realisations of the $\left.f B M B^{(H)}\right)$. We may take

$$
\sigma^{2}=\ell^{4+2 H} \times \frac{2^{1+H} \Gamma\left(H+\frac{5}{2}\right)}{\sqrt{\pi}} \times \frac{H}{2+7 H+7 H^{2}+2 H^{3}} \times \int_{-\infty}^{\infty} \varphi_{c}(x)^{2} \pi\left(x \mid B^{(H)} ; c\right) \mathrm{d} x .
$$

We emphasise that here the log marginal target density is twice differentiable and $H$ measures the roughness of the second derivative as noted below. (In the RWM case $H$ measures the roughness of the log marginal target density itself.)

Again, the anomalous rate means that the optimal scaling of [30] cannot here apply, moreover that the rate of proposal variance decay is optimal. Again optimising over the choice of $\ell$ leads to different optimal acceptance rates for different values of $H$. As exhibited in the right panel of Figure 1, and as in the RWM case, the optimal acceptance rate increases as $H$ increases. In the MALA case $H$ measures the roughness of the second derivative of the target (so $\pi$ is " $2+H$ smooth") and the optimal acceptance rate does not decay to zero as $H$ does. In fact both plots of Figure 1 are obtained by numerically solving the same equation (see Section 8.1) over different ranges of smoothness parameter, $(0,1)$ for RWM and $(2,3)$ MALA. The choice of optimal acceptance rate also seems to be much more robust in case of MALA; this is supported by the numerical examples in Section 8.3.

The marginal target probability densities for these counterexamples are chosen to facilitate simple proofs; many other constructions work equally well. The RWM choice is a fractional Brownian perturbation of a normal density; the MALA choice is based on a fractional Brownian perturbation at the level of the second derivative of the log-density, so

$$
\left(\frac{d}{\mathrm{~d} x}\right)^{2}\left(\log \left(\pi\left(x \mid B^{(H)} ; c\right)\right)\right)=-1+\varphi_{c}(x) B^{(H)} .
$$

The proofs will work for other kinds of perturbation, and indeed it is an interesting question what exactly are the analytical features of a marginal target probability density that would lead to anomalous scaling.

In this paper we do not proceed to establish weak convergence to Langevin diffusion limits, because the current results are sufficient to establish counterexamples. This and other related questions are further discussed in Section 8.2. Section 8.3 discussed the question of what useful heuristics can be learned from these results. 
3. Generalities concerning Metropolis-Hastings log acceptance rates. In this section we describe a general framework for proving CLT-type results such as Theorems 1 and 2. The treatment follows the style of Tierney [33], and applies to rather general Metropolis-Hastings (MH) samplers.

Let $(\mathcal{X}, \mathcal{F})$ be a measurable space, supporting a probability measure $\pi$ (the "target probability distribution") and a Markov kernel $(Q(x, \cdot): x \in \mathcal{X})$ (the "proposal mechanism"). Recall that $(Q(x, \cdot): x \in \mathcal{X})$ is a Markov kernel if (i) $x \mapsto Q(x, A)$ is measurable for any $A \in \mathcal{F}$ and (ii) $B \mapsto Q(x, B)$ is a probability measure on $(\mathcal{X}, \mathcal{F})$ for any $x \in \mathcal{X}$. As described by Tierney [33], Proposition 1 , let $\mathcal{R} \in \mathcal{F} \otimes \mathcal{F}$ be the symmetric set such that $\pi(\mathrm{d} x) Q(x, \mathrm{~d} y)$ and $\pi(\mathrm{d} y) Q(y, \mathrm{~d} x)$ are mutually absolutely continuous on $\mathcal{R}$ and mutually singular off $\mathcal{R}$. Tierney notes that $\mathcal{R}$ is unique up to differences of sets which are null with respect to both these measures.

We define the $\log \mathrm{MH}$ acceptance ratio (log-MH-ratio) $\rho$ by

$$
\rho(x, y)= \begin{cases}\log \left(\frac{\pi(\mathrm{d} y) Q(y, \mathrm{~d} x)}{\pi(\mathrm{d} x) Q(x, \mathrm{~d} y)}\right) & \text { if }(x, y) \in \mathcal{R}, \\ 0 & \text { otherwise. }\end{cases}
$$

We write $\rho$ for the random variable $\rho(X, Y)$, where $(X, Y)$ has distribution given by $\pi(\mathrm{d} x) Q(x, \mathrm{~d} y)$.

It is straightforward to verify that the definition of $\rho$ and the reversibility of the MH algorithm under $\pi$ together imply the following computational relationships.

Proposition 3. With $\rho(x, y)$ defined as above:

3.(a) $\rho(x, y)=-\rho(y, x)$ almost everywhere with respect to $\pi(\mathrm{d} x) Q(x, \mathrm{~d} y)$.

3.(b) Let $f: \mathbb{R} \rightarrow \mathbb{R}$ be a measurable function such that $\mathbb{E}[|f(\rho)|]<\infty$, so that $f \circ \rho$ is integrable with respect to $\pi(\mathrm{d} x) Q(x, \mathrm{~d} y)$. Then $\mathbb{E}\left[f(-\rho) e^{\rho}\right]=\mathbb{E}[f(\rho)]$.

Proof. By Radon-Nikodym theorem 3.(a) is immediate from (4) and the symmetry of $\mathcal{R}$.

To establish Proposition 3.(b), argue as follows. Using 3.(a), we know that $f(-\rho) e^{\rho} \mathbb{I}[\rho \leq$ $\kappa]$ will be integrable against the probability measure $\pi(\mathrm{d} x) Q(x, \mathrm{~d} y)$ for each positive constant $\kappa$, hence

$$
\begin{aligned}
\mathbb{E}[ & \left.f(-\rho) e^{\rho} ; \rho \leq \kappa\right] \\
= & \iint_{\rho(x, y) \leq \kappa} f(-\rho(x, y)) e^{\rho(x, y)} \pi(\mathrm{d} x) Q(x, \mathrm{~d} y) \\
= & \iint_{(x . y) \in \mathcal{R} \text { and } \rho(x, y) \leq \kappa} f(-\rho(x, y)) e^{\rho(x, y)} \pi(\mathrm{d} x) Q(x, \mathrm{~d} y)+f(0) \iint_{\mathcal{R}^{c}} \pi(\mathrm{d} x) Q(x, \mathrm{~d} y) \\
= & \iint_{(x . y) \in \mathcal{R} \text { and } \rho(x, y) \leq \kappa} f(-\rho(x, y)) \frac{\pi(\mathrm{d} y) Q(y, \mathrm{~d} x)}{\pi(\mathrm{d} x) Q(x, \mathrm{~d} y)} \pi(\mathrm{d} x) Q(x, \mathrm{~d} y) \\
& +f(0) \iint_{\mathcal{R}^{c}} \pi(\mathrm{d} x) Q(x, \mathrm{~d} y) \\
= & \iint_{(x . y) \in \mathcal{R} \text { and } \rho(x, y) \leq \kappa} f(-\rho(x, y)) \pi(\mathrm{d} y) Q(y, \mathrm{~d} x)+f(0) \iint_{\mathcal{R}^{c}} \pi(\mathrm{d} y) Q(y, \mathrm{~d} x) \\
= & \iint_{\rho(y, x) \geq-\kappa} f(\rho(y, x)) \pi(\mathrm{d} y) Q(y, \mathrm{~d} x)=\mathbb{E}[f(\rho) ; \rho \geq-\kappa] .
\end{aligned}
$$

The fact that $\mathcal{R}$ is a symmetric set is used for the fourth equality, while Proposition 3.(a) is used for the fifth. 
Applying the above to the function $|f|$, and letting $\kappa \rightarrow \infty$, yields $\mathbb{E}\left[|f(-\rho)| e^{\rho}\right]=$ $\mathbb{E}[|f(\rho)|]<\infty$ by monotone convergence. Hence $\mathbb{E}\left[f(-\rho) e^{\rho} ; \rho \leq \kappa\right] \rightarrow \mathbb{E}\left[f(-\rho) e^{\rho}\right]$, by dominated convergence. Proposition 3.(b) then follows by letting $\kappa \rightarrow \infty$ in the equation above.

REMARK 4. Useful identities following from Proposition 3 include:

4.(a) $\mathbb{E}\left[e^{\rho}\right]=1$;

4.(b) $\mathbb{E}\left[f(\rho) e^{\rho / 2}\right]=\mathbb{E}\left[f(-\rho) e^{\rho / 2}\right]$ whenever $f(\rho) e^{\rho / 2}$ is an integrable random variable;

4.(c) $\mathbb{E}\left[f(\rho) e^{\rho / 2}\right]=0$ whenever $f$ is an odd function and $f(\rho) e^{\rho / 2}$ is an integrable random variable.

4.(d) If $f$ is an even function and $f(\rho)$ is an integrable random variable then $f(\rho) e^{t \rho}$ is an integrable random variable for all $t \in(0,1)$.

Typically, when establishing optimal scaling results, a key task is to determine when it is valid to assert asymptotically negligibility of half the variance of $\rho$ plus its mean. The next few results establish when this asymptotically negligibility holds for a rather general context.

Proposition 5. Suppose that $\mathbb{E}\left[\rho^{2}\right]<\infty$. Then $\mathbb{E}[\rho]+\frac{1}{2} \mathbb{E}\left[\rho^{2}\right]=\mathbb{E}\left[\rho^{2} \int_{0}^{\frac{1}{2}}\left(1-e^{t \rho}\right) \mathrm{d} t\right]$.

Proof. Consider the exact Taylor expansion (valid for all values of $\rho$ )

$$
e^{\rho}=1+\rho+\frac{1}{2} \rho^{2}+\rho^{2} \int_{0}^{1}(1-t)\left(e^{t \rho}-1\right) \mathrm{d} t .
$$

Taking expectations and using Remark 4.(a),

$$
\mathbb{E}\left[\rho+\rho^{2} / 2\right]=\mathbb{E}\left[\rho^{2} \int_{0}^{1}(1-t)\left(1-e^{t \rho}\right) \mathrm{d} t\right] .
$$

The proof is concluded by the following sequence of equalities. They are justified respectively by applying the Fubini-Tonelli theorem for exchanging order of integral when the integrand is bounded above, using Proposition 3.(b) for the function $f(\rho)=\rho^{2} e^{t \rho}$ (which is integrable by Remark 4.(d)), changing variables using $u=1-t$, and finally applying the Fubini-Tonelli theorem once more:

$$
\begin{aligned}
\mathbb{E} & {\left[\rho^{2} \int_{\frac{1}{2}}^{1}(1-t)\left(1-e^{t \rho}\right) \mathrm{d} t\right] } \\
& =\int_{\frac{1}{2}}^{1}(1-t) \mathbb{E}\left[\rho^{2}\left(1-e^{t \rho}\right)\right] \mathrm{d} t=\int_{\frac{1}{2}}^{1}(1-t) \mathbb{E}\left[\rho^{2}\left(1-e^{(1-t) \rho}\right)\right] \mathrm{d} t \\
& =\int_{0}^{\frac{1}{2}} u \mathbb{E}\left[\rho^{2}\left(1-e^{u \rho}\right)\right] \mathrm{d} u=\mathbb{E}\left[\rho^{2} \int_{0}^{\frac{1}{2}} u\left(1-e^{u \rho}\right) \mathrm{d} u\right] .
\end{aligned}
$$

We now establish a bound on the right-hand side $\mathbb{E}\left[\rho^{2} \int_{0}^{\frac{1}{2}}\left(1-e^{t \rho}\right) \mathrm{d} t\right]$ of Proposition 5 which will be sufficient for our purposes.

Lemma 6. Suppose that $\mathbb{E}\left[\rho^{2}\right]<\infty$. For every $\kappa>0$ the following bound holds:

$$
\left|\mathbb{E}\left[\rho^{2} \int_{0}^{\frac{1}{2}}\left(1-e^{t \rho}\right) \mathrm{d} t\right]\right|<\sinh \left(\frac{1}{2} \kappa\right) \mathbb{E}\left[\rho^{2}\right]+\mathbb{E}\left[\rho^{2} ; \rho<-\kappa\right] .
$$


PROOF. Fix $\kappa>0$ and split the above integral into parts according to whether $\rho \in$ $[-\kappa, \kappa], \rho<-\kappa$ or $\rho>\kappa$. If $\rho \in[-\kappa, \kappa]$, then $\left|\int_{0}^{\frac{1}{2}}\left(1-e^{t \rho}\right) \mathrm{d} t\right| \leq \frac{1}{2} \max \left\{1-e^{-\frac{1}{2} \kappa}, e^{\frac{1}{2} \kappa}-\right.$ $1\}=\frac{1}{2}\left(e^{\frac{1}{2} \kappa}-1\right)$. Using $\frac{1}{2}\left(e^{\frac{1}{2} \kappa}-1\right)<\sinh \left(\frac{1}{2} \kappa\right)$ for the sake of simplicity, we obtain

$$
\left|\mathbb{E}\left[\rho^{2} \int_{0}^{\frac{1}{2}}\left(1-e^{t \rho}\right) \mathrm{d} t ;-\kappa \leq \rho^{2} \leq \kappa\right]\right|<\sinh \left(\frac{1}{2} \kappa\right) \mathbb{E}\left[\rho^{2}\right] .
$$

If $\rho<-\kappa$, then $1-e^{t \rho}<1$ so $\left|\mathbb{E}\left[\rho^{2} \int_{0}^{\frac{1}{2}}\left(1-e^{t \rho}\right) \mathrm{d} t ; \rho<-\kappa\right]\right|<\frac{1}{2} \mathbb{E}\left[\rho^{2} ; \rho<-\kappa\right]$. Similarly, if $\rho>\kappa$, then $e^{t \rho}-1<e^{\rho}$. Applying Proposition 3.(b) to the function $f(\rho)=\rho^{2} \mathbb{I}[\kappa<$ $\rho]$, this implies

$$
\left|\mathbb{E}\left[\rho^{2} \int_{0}^{\frac{1}{2}}\left(1-e^{t \rho}\right) \mathrm{d} t ; \kappa<\rho\right]\right|<\frac{1}{2} \mathbb{E}\left[\rho^{2} e^{\rho} ; \kappa<\rho\right]=\frac{1}{2} \mathbb{E}\left[\rho^{2} ; \rho<-\kappa\right] .
$$

The result follows by adding these three terms.

Now consider a sequence of log-MH-ratios $\rho_{n}$, possibly defined on different probability spaces and associated with different target probability distributions $\pi_{n}$, proposal kernels $Q_{n}$ and derived log-MH-ratios $\rho_{n}$. Asymptotic negligibility of the second moment of $\rho_{n}$ and a technical condition weaker than the uniform integrability of the scaled random variables $\rho_{n}^{2} / \mathbb{E}\left[\rho_{n}^{2}\right]$ on $\rho_{n}<0$, imply that mean plus half variance of $\rho_{n}$ is asymptotically negligible:

THEOREM 7 ("Half variance plus mean is asymptotically negligible"). Suppose that $\mathbb{E}\left[\rho_{n}^{2}\right] \rightarrow 0$ as $n \rightarrow \infty$, and suppose moreover we can find positive constants $\kappa_{n} \rightarrow 0$ such that $\mathbb{E}\left[\rho_{n}^{2} ; \rho_{n}<-\kappa_{n}\right]=o\left(\mathbb{E}\left[\rho_{n}^{2}\right]\right)$ as $n \rightarrow \infty$. Then $\mathbb{E}\left[\rho_{n}\right]+\frac{1}{2} \mathbb{E}\left[\rho_{n}^{2}\right]=o\left(\mathbb{E}\left[\rho_{n}^{2}\right]\right)$ as $n \rightarrow \infty ;$ indeed

$$
\left|\mathbb{E}\left[\rho_{n}\right]+\frac{1}{2} \mathbb{E}\left[\rho_{n}^{2}\right]\right| \leq \sinh \left(\frac{1}{2} \kappa_{n}\right) \mathbb{E}\left[\rho_{n}^{2}\right]+\mathbb{E}\left[\rho_{n}^{2} ; \rho_{n}<-\kappa_{n}\right]=o\left(\mathbb{E}\left[\rho_{n}^{2}\right]\right) .
$$

Moreover

$$
\mathbb{E}\left[\rho_{n}\right]+\frac{1}{2} \operatorname{Var}\left[\rho_{n}\right]=o\left(\mathbb{E}\left[\rho_{n}^{2}\right]\right)
$$

PROOF. The inequality follows directly from Proposition 5 and Lemma 6. The asymptotic negligibility of mean plus half variance follows from the observation that $\operatorname{Var}\left[\rho_{n}\right]=$ $\mathbb{E}\left[\rho_{n}^{2}\right]-\mathbb{E}\left[\rho_{n}\right]^{2}$ : we control $\mathbb{E}\left[\rho_{n}\right]^{2}$ by iterating the argument, since $\left(\mathbb{E}\left[\rho_{n}\right]\right)^{2}=\left(o\left(\mathbb{E}\left[\rho_{n}^{2}\right]\right)-\right.$ $\left.\frac{1}{2} \mathbb{E}\left[\rho_{n}^{2}\right]\right)^{2}=o\left(\mathbb{E}\left[\rho_{n}^{2}\right]\right.$ ) (expanding the quadratic and using $\mathbb{E}\left[\rho_{n}^{2}\right] \rightarrow 0$ ).

We now establish a central limit theorem for suitable sums of independent log-MetropolisHastings ratios (as would arise when considering suitable product-distribution targets). The main requirement is simply a particular uniform integrability condition on the sequence of scaled squares of the log-Metropolis-Hastings ratios, corresponding to a Lindeberg condition.

THEOREM 8. Consider a triangular array formed by $\rho_{n, j}\left(\right.$ for $j=1, \ldots, m_{n}, n=$ $1,2, \ldots)$, built out of row-wise independent log-Metropolis-Hastings-ratio random variables for targets $\pi_{n j}$ and proposals $q_{n j}$. Suppose that there exists a sequence of positive numbers $\kappa_{n, j}$ such that $\lim _{n \rightarrow \infty} \sup _{j \leq m_{n}} \kappa_{n, j}=0$, and $\mathbb{E}\left[\rho_{n, j}^{2} ; \rho_{n, j}<-\kappa_{n, j}\right]=o\left(\mathbb{E}\left[\rho_{n, j}^{2}\right]\right)$ uniformly, in the sense that

$$
\lim _{n \rightarrow \infty} \sup _{j \leq m_{n}} \frac{\mathbb{E}\left[\rho_{n, j}^{2} ; \rho_{n, j}<-\kappa_{n, j}\right]}{\mathbb{E}\left[\rho_{n, j}^{2}\right]}=0
$$


Suppose further that there exists a constant $\sigma^{2}<\infty$ such that $\lim _{n \rightarrow \infty} \sum_{j=1}^{m_{n}} \mathbb{E}\left[\rho_{n j}^{2}\right]=\sigma^{2}$. Then

$$
\sum_{j=1}^{m_{n}} \rho_{n, j} \stackrel{w}{\rightarrow} N\left(-\frac{1}{2} \sigma^{2}, \sigma^{2}\right)
$$

PRoof. The Lindeberg central limit theorem (see, e.g., [15], Theorem 4.15) follows from the following conditions:

(i) $\sum_{j=1}^{m_{n}} \mathbb{P}\left[\left|\rho_{n, j}\right|>\varepsilon\right] \rightarrow 0$ for any $\varepsilon>0$,

(ii) $\sum_{j=1}^{m_{n}} \mathbb{E}\left[\rho_{n, j} ;\left|\rho_{n, j}\right| \leq 1\right] \rightarrow-\frac{1}{2} \sigma^{2}$,

(iii) $\sum_{j=1}^{m_{n}} \operatorname{Var}\left[\rho_{n, j} ;\left|\rho_{n, j}\right| \leq 1\right] \rightarrow \sigma^{2}$.

Note that Proposition 3.(b) implies that, for any nonnegative function $f$ and for any $n, j$, $\mathbb{E}\left[f\left(\left|\rho_{n, j}\right|\right) ; \rho_{n, j}>\kappa_{n, j}\right] \leq \mathbb{E}\left[f\left(\left|\rho_{n, j}\right|\right) e^{\rho_{n, j}} ; \rho_{n, j}>\kappa_{n, j}\right]=\mathbb{E}\left[f\left(\left|\rho_{n, j}\right|\right) ; \rho_{n, j}<-\kappa_{n, j}\right]$, and hence

$$
\mathbb{E}\left[f\left(\left|\rho_{n, j}\right|\right) ;\left|\rho_{n, j}\right|>\kappa_{n, j}\right] \leq 2 \mathbb{E}\left[f\left(\left|\rho_{n, j}\right|\right) ; \rho_{n, j}<-\kappa_{n, j}\right] .
$$

Taking $f(x)=x^{2}$ in (6) yields the following for fixed $\varepsilon>0$ and for all sufficiently large integers $n$ :

$$
\begin{aligned}
\sum_{j=1}^{m_{n}} \mathbb{P}\left[\rho_{n, j}^{2}>\varepsilon^{2}\right] & \leq \frac{1}{\varepsilon^{2}} \sum_{j=1}^{m_{n}} \mathbb{E}\left[\rho_{n, j}^{2} ;\left|\rho_{n, j}\right|>\varepsilon\right] \leq \frac{1}{\varepsilon^{2}} \sum_{j=1}^{m_{n}} \mathbb{E}\left[\rho_{n, j}^{2} ;\left|\rho_{n, j}\right|>\kappa_{n, j}\right] \\
& \leq \frac{2}{\varepsilon^{2}} \sum_{j=1}^{m_{n}} \mathbb{E}\left[\rho_{n, j}^{2} ; \rho_{n, j}<-\kappa_{n, j}\right] \\
& \leq \frac{2}{\varepsilon^{2}}\left(\sum_{j=1}^{m_{n}} \mathbb{E}\left[\rho_{n, j}^{2}\right]\right) \times \sup _{j \leq m_{n}} \frac{\mathbb{E}\left[\rho_{n, j}^{2} ; \rho_{n, j}<-\kappa_{n, j}\right]}{\mathbb{E}\left[\rho_{n, j}^{2}\right]} .
\end{aligned}
$$

But we have supposed that $\sum_{j=1}^{m_{n}} \mathbb{E}\left[\rho_{n j}^{2}\right] \rightarrow \sigma^{2}<\infty$, so CLT requirement (i) follows from (5). 0 .

Note $\mathbb{E}\left[\rho_{n, j} ;\left|\rho_{n, j}\right|>1\right] \leq \mathbb{E}\left[\rho_{n, j}^{2} ;\left|\rho_{n, j}\right|>1\right]$. It follows that $\sum_{j=1}^{m_{n}} \mathbb{E}\left[\rho_{n, j} ;\left|\rho_{n, j}\right|>1\right] \rightarrow$ Note also $\operatorname{Var}\left[\rho_{n, j} ;\left|\rho_{n, j}\right|>1\right] \leq \mathbb{E}\left[\rho_{n, j}^{2} ;\left|\rho_{n, j}\right|>1\right]$ and therefore it also follows that

$$
\sum_{j=1}^{m_{n}} \operatorname{Var}\left[\rho_{n, j} ;\left|\rho_{n, j}\right|>1\right] \rightarrow 0 .
$$

Proposition 5 and Lemma 6 imply the asymptotic relationship

$$
\begin{aligned}
& \sum_{j=1}^{m_{n}}\left|\mathbb{E}\left[\rho_{n, j}\right]+\frac{1}{2} \mathbb{E}\left[\rho_{n, j}^{2}\right]\right| \\
& \quad \leq \sum_{j=1}^{m_{n}}\left(\sinh \left(\frac{1}{2} \kappa_{n, j}\right) \mathbb{E}\left[\rho_{n, j}^{2}\right]+\mathbb{E}\left[\rho_{n, j}^{2} ; \rho_{n, j}<-\kappa_{n, j}\right]\right) \\
& \quad \leq\left(\sinh \left(\frac{1}{2} \sup _{j \leq m_{n}} \kappa_{n, j}\right)+\sup _{j \leq m_{n}} \frac{\mathbb{E}\left[\rho_{n, j}^{2} ; \rho_{n, j}<-\kappa_{n, j}\right]}{\mathbb{E}\left[\rho_{n, j}^{2}\right]}\right) \sum_{j=1}^{m_{n}} \mathbb{E}\left[\rho_{n, j}^{2}\right] \\
& \quad \rightarrow 0 .
\end{aligned}
$$


The combination of $\sup _{j \leq m_{n}} \kappa_{n, j} \rightarrow 0$ and $\sum_{j=1}^{m_{n}} \mathbb{E}\left[\rho_{n j}^{2}\right] \rightarrow \sigma^{2}$ and (5) together imply convergence to 0 .

Hence CLT requirement (ii) follows, since $\sum_{j=1}^{m_{n}} \mathbb{E}\left[\rho_{n, j} ;\left|\rho_{n, j}\right|>1\right] \rightarrow 0$, together with the asymptotic relationship (8), implies that

$$
\lim _{n \rightarrow \infty} \sum_{j=1}^{m_{n}} \mathbb{E}\left[\rho_{n, j} ;\left|\rho_{n, j}\right| \leq 1\right]=\lim _{n \rightarrow \infty} \sum_{j=1}^{m_{n}} \mathbb{E}\left[\rho_{n, j}\right]=-\frac{1}{2} \lim _{n \rightarrow \infty} \sum_{j=1}^{m_{n}} \mathbb{E}\left[\rho_{n, j}^{2}\right]=-\frac{\sigma^{2}}{2} .
$$

Finally, we deal with CLT requirement (iii). First note that inequality (6) implies $\mathbb{E}\left[\rho_{n, j}^{2}\right] \leq$ $\kappa_{n, j}^{2}+2 \mathbb{E}\left[\rho_{n, j}^{2} ; \rho_{n, j}<-\kappa_{n, j}\right]$ for every $n, j$. Hence $\lim _{n \rightarrow \infty} \sup _{j \leq m_{n}} \kappa_{n, j}=0$ and the uniform integrability together imply that

$$
\lim _{n \rightarrow \infty} \sup _{j \leq m_{n}} \mathbb{E}\left[\rho_{n, j}^{2}\right]=0 .
$$

Thus the asymptotic relationship (8) allows us to deduce that as $n \rightarrow \infty$ so

$$
\begin{aligned}
\lim _{n \rightarrow \infty} \sum_{j=1}^{m_{n}} \mathbb{E}\left[\rho_{n, j}\right]^{2} & =\lim _{n \rightarrow \infty} \frac{1}{4} \sum_{j=1}^{m_{n}} \mathbb{E}\left[\rho_{n, j}^{2}\right]^{2} \leq \lim _{n \rightarrow \infty} \frac{1}{4} \sum_{j^{\prime}=1}^{m_{n}} \mathbb{E}\left[\rho_{n, j^{\prime}}^{2}\right] \cdot \sup _{j \leq m_{n}} \mathbb{E}\left[\rho_{n, j}^{2}\right] \\
& =\frac{\sigma^{2}}{4} \lim _{n \rightarrow \infty} \sup _{j \leq m_{n}} \mathbb{E}\left[\rho_{n, j}^{2}\right]=0 .
\end{aligned}
$$

This implies CLT requirement (iii) as follows: using (7),

$$
\begin{aligned}
\lim _{n \rightarrow \infty} \sum_{j=1}^{m_{n}} \operatorname{Var}\left[\rho_{n, j} ;\left|\rho_{n, j}\right| \leq 1\right] & =\lim _{n \rightarrow \infty} \sum_{j=1}^{m_{n}} \operatorname{Var}\left[\rho_{n, j}\right] \\
& =\lim _{n \rightarrow \infty}\left(\left(\sum_{j=1}^{m_{n}} \mathbb{E}\left[\rho_{n, j}^{2}\right]\right)-\left(\sum_{j=1}^{m_{n}} \mathbb{E}\left[\rho_{n, j}\right]^{2}\right)\right) \\
& =\lim _{n \rightarrow \infty} \sum_{j=1}^{m_{n}} \mathbb{E}\left[\rho_{n, j}^{2}\right]=\sigma^{2} .
\end{aligned}
$$

REMARK 9. Note that asymptotic as $n \rightarrow \infty$ the second moment $\mathbb{E}\left[\rho_{n, j}^{2}\right]$ is uniformly negligible (in $j$ ). This is established by (9) as a consequence of the assumptions of Theorem 8 .

REMARK 10. The fundamental difference between this central limit theorem and those established in Roberts, Gelman and Gilks [29] and all subsequent optimal scaling results is as follows. Our result is not conditional on a specific target location. It concerns random variables that are simultaneously dependent on the target and the proposal draw, as opposed to showing that for most fixed target draws the log Metropolis-Hastings ratios (viewed only as functions of the proposal) satisfy a central limit theorem. This subtle difference allows the use of weaker smoothness conditions.

4. Variations on Isserlis theorem. We seek an analysis of optimal scaling for RWM and MALA when the marginal target probability density function depends on the Gaussian random process given by the two-sided fBM $B^{(H)}$, as prescribed in Theorems 1 and 2 . This analysis requires a variation on the classical result of Isserlis [11], and consequent estimates and computations, which we now describe.

First we introduce some preliminary combinatorial notation. Given a multiset $S$, a pairing is a partition of $S$ into pairs (each pair possibly containing the same element twice). A pairing 
is called proper if each of its pairs contains two distinct elements. Let $\mathcal{P}(S)$ denote the set of all pairings of the multiset $S$, and let $\mathcal{P}^{*}(S) \subseteq \mathcal{P}(S)$ denote the set of all proper pairings. Isserlis' theorem, sometimes also called Wick's theorem runs as follows:

THEOREM 11 ([11]). Let $X=\left(X_{1}, X_{2}, \ldots, X_{n}\right)$ be centred multivariate normal random variable and consider a general multiset $S=\left\{s_{1}, s_{2}, \ldots, s_{m}\right\}$, with $s_{i} \in\{1,2, \ldots, n\}$ for $i=$ $1, \ldots, m$. Then

$$
\mathbb{E}\left[X_{s_{1}} X_{s_{2}} \cdots X_{s_{m}}\right]= \begin{cases}\sum_{p \in \mathcal{P}(S)} \prod_{\Lambda \in p} \mathbb{E}\left[X_{\lambda_{1}} X_{\lambda_{2}}\right] & \text { if } m \text { is even }, \\ 0 & \text { if } m \text { is odd }\end{cases}
$$

where the product is taken over all pairs $\Lambda=\left\{\lambda_{1}, \lambda_{2}\right\}$ of a pairing $p$.

A proof can be found, for example, in Janson [12], Theorem 1.28.

Isserlis' theorem leads to the following proposition.

PROPOSITION 12. Let $X=\left(X_{1}, X_{2}, \ldots, X_{n+k}\right)$ be a centred multivariate normal random variable with covariance matrix $R$ and let $S=2 \times\{1,2, \ldots, n\} \cup\{n+1, n+2, \ldots$, $n+k\}$, using a compact multiset notation to signal that elements $1, \ldots, n$ appear twice while elements $n+1, \ldots, n+k$ appear once only. Then

$$
\begin{aligned}
& \mathbb{E}\left[\left(X_{1}^{2}-R_{11}\right)\left(X_{2}^{2}-R_{22}\right) \cdots\left(X_{n}^{2}-R_{n n}\right) X_{n+1} X_{n+2} \cdots X_{n+k}\right] \\
& \quad= \begin{cases}\sum_{p \in \mathcal{P}^{*}(S)} \prod_{\Lambda \in p} \mathbb{E}\left[X_{\lambda_{1}} X_{\lambda_{2}}\right] & \text { for even } k, \\
0 & \text { for odd } k .\end{cases}
\end{aligned}
$$

Note the crucial difference between expansions in Theorem 11 and Proposition 12: in the proposition the sum is taken over the set $\mathcal{P}^{*}$ of proper pairings.

PROOF. It suffices to consider the result when the covariance matrix $R$ lies in the interior of the set of all valid covariance matrices: the general result then follows by a continuity argument. This allows us to argue algebraically, viewing relevant expectations as multivariate polynomials in the entries of $R$.

First note that the result follows trivially if $k$ is odd: apply inclusion-exclusion of $R_{11}, R_{22}, \ldots, R_{n n}$ to

$$
\mathbb{E}\left[\left(X_{1}^{2}-R_{11}\right)\left(X_{2}^{2}-R_{22}^{2}\right) \cdots\left(X_{n}^{2}-R_{n n}\right) X_{n+1} X_{n+2} \cdots X_{n+k}\right] .
$$

In case of odd $k$, each term in the inclusion-exclusion expansion must vanish by Theorem 11. So we need consider only the case of even $k$.

Consider the Isserlis expansion of $I_{1}=\mathbb{E}\left[X_{1}^{2} X_{2}^{2} \cdots X_{n}^{2} \cdot X_{n+1} X_{n+2} \cdots X_{n+k}\right]$, viewed as a sum of monomials in the entries of $R$. According to the combinatorial expression for this given in Theorem 11, if we remove all monomials involving any of $R_{11}, R_{22}, \ldots, R_{n n}$ then the remaining sum is exactly $\sum_{p \in \mathcal{P}^{*}(S)} \prod_{\Lambda \in p} \mathbb{E}\left[X_{\lambda_{1}} X_{\lambda_{2}}\right]$.

Now consider the Isserlis expansion of $I_{2}=\mathbb{E}\left[\left(X_{1}^{2}-R_{11}\right)\left(X_{2}^{2}-R_{22}\right) \cdots\left(X_{n}^{2}-R_{n n}\right) \times\right.$ $\left.X_{n+1} \cdots X_{n+k}\right]$, viewed as a linear combination of monomials of $X_{1}, X_{2}, \ldots, X_{n+k}$. This agrees with the Isserlis expansion of $I_{1}$ up to a difference of a linear combination of monomials involving nonempty selections of $R_{11}, R_{22}, \ldots, R_{n n}$.

The result is therefore proved if we can establish that, after cancellation, the expansion of $I_{2}$ contains no terms involving any of the diagonal entries $R_{11}, R_{22}, \ldots, R_{n n}$. 
The joint moment generating function of the multivariate normal variable $X$ equals $\exp \left(\frac{1}{2} t^{\top} R t\right)$, for $t \in \mathbb{R}^{n+k}$. Hence

$$
I_{2}=\left[\left(\frac{\partial^{2}}{\partial t_{1}^{2}}-R_{11}\right) \ldots\left(\frac{\partial^{2}}{\partial t_{n}^{2}}-R_{n n}\right) \frac{\partial}{\partial t_{n+1}} \ldots \frac{\partial}{\partial t_{n+k}} \exp \left(\frac{1}{2} t^{\top} R t\right)\right]_{t_{1}=0, \ldots, t_{n+k}=0} .
$$

Viewing this as a smooth function of the vector $t$ and the entries of $R$ and denoting the differential operator $D=\left(\frac{\partial^{2}}{\partial t_{2}^{2}}-R_{22}\right) \ldots\left(\frac{\partial^{2}}{\partial t_{n}^{2}}-R_{n n}\right) \frac{\partial}{\partial t_{n+1}} \ldots \frac{\partial}{\partial t_{n+k}}$ observe that

$$
\begin{aligned}
& \frac{\partial}{\partial R_{11}} \mathbb{E}\left[\left(X_{1}^{2}-R_{11}\right) \cdots\left(X_{n}^{2}-R_{n n}\right) X_{n+1} \cdots X_{n+k}\right] \\
& \quad=\frac{\partial}{\partial R_{11}}\left[\left(\frac{\partial^{2}}{\partial t_{1}^{2}}-R_{11}\right) D \exp \left(\frac{1}{2} t^{\top} R t\right)\right]_{t_{1}=0, \ldots, t_{n+k}=0} \\
& \quad=\left[D \frac{\partial}{\partial R_{11}}\left(\frac{\partial^{2}}{\partial t_{1}^{2}}-R_{11}\right) \exp \left(\frac{1}{2} t^{\top} R t\right)\right]_{t_{1}=0, \ldots, t_{n+k}=0} \\
& \quad=\left[D \frac{\partial}{\partial R_{11}}\left(\sum_{i=1}^{n+k} R_{1 i} t_{i}\right)^{2} \exp \left(\frac{1}{2} t^{\top} R t\right)\right]_{t_{1}=0, \ldots, t_{n+k}=0} \\
& \quad=\left[t_{1} D \exp \left(\frac{1}{2} t^{\top} R t\right)\left(t_{1}\left(\sum_{i=1}^{n+k} R_{1 i} t_{i}\right)^{2}+2 \sum_{i=1}^{n+k} R_{1 i} t_{i}\right)\right]_{t_{1}=0, \ldots, t_{n+k}=0}=0 .
\end{aligned}
$$

The second identity holds because we can swap the order of differentiation and interchange differentiation with taking the limit $t_{i} \rightarrow 0$. This is justified since $\exp \left(\frac{1}{2} t^{\top} R t\right)$ and all its derivatives are smooth and for a smooth function $f$ the functions $R_{11} \mapsto f\left(R_{11}, t\right)$ converge uniformly to $R_{11} \mapsto f\left(R_{11}, 0\right)$ (as $t \rightarrow 0$ ) in some compact neighbourhood of $\left(R_{11}, 0\right)$.

The same argument applies for differentiation with respect to $R_{22}, \ldots, R_{n n}$. Thus it follows that $I_{2}$ is free of all terms involving $R_{11}, R_{22}, \ldots, R_{n n}$, hence must equal $\sum_{p \in \mathcal{P}^{*}(S)} \prod_{\Lambda \in p} \mathbb{E}\left[X_{\lambda_{1}} X_{\lambda_{2}}\right]$ as required.

LEMMA 13. Let $X=\left(X_{0}, X_{1}, X_{2}, \ldots, X_{n}\right)$ be a centred multivariate normal random variable with covariance matrix $R$. Then

$$
\begin{aligned}
& \mathbb{E}\left[\exp \left(X_{0}\right) \times \prod_{i=1}^{n}\left(X_{i}^{2}-R_{i i}\right)\right] \\
& \quad=\exp \left(\frac{1}{2} R_{00}\right) \times \mathbb{E}\left[\prod_{i=1}^{n}\left(\left(X_{i}+R_{0 i}\right)^{2}-R_{i i}\right)\right] \\
& \quad=\exp \left(\frac{1}{2} R_{00}\right) \sum_{\substack{A_{1} \sqcup A_{2} \sqcup A_{3}=\{1,2, \ldots, n\} \\
\left|A_{2}\right| \text { is even }}} \sum_{p \in \mathcal{P}^{*}\left(2 A_{1} \cup A_{2}\right)} 2^{\left|A_{2}\right|} \prod_{i \in A_{2}} R_{0 i} \times \prod_{i \in A_{3}} R_{0 i}^{2} \times \prod_{\Lambda \in p} R_{\lambda_{1} \lambda_{2}},
\end{aligned}
$$

where the inner sum ranges over $\mathcal{P}^{*}\left(2 A_{1} \cup A_{2}\right)$, the set of all proper pairings of the multiset $2 A_{1} \cup A_{2}$ in which elements of $A_{1}$ appear twice and elements of $A_{2}$ appear once. The outer sum ranges over all three-fold partitions $A_{1}, A_{2}, A_{3}$ of the set $\{1,2, \ldots, n\}$ with $A_{2}$ containing evenly many elements.

ProOF. We may suppose that $X=C^{\top} Z$, where $Z$ is a $k$-dimensional standard normal random variable and $C$ is a $k \times(n+1)$ matrix. Thus the covariance matrix of $X$ is $R=$ $\mathbb{E}\left[X X^{\top}\right]=\mathbb{E}\left[C^{\top} Z Z^{\top} C\right]=C^{\top} C$, and $X_{i}=e_{i}^{\top} C^{\top} Z$ where $e_{i}$ is an $(n+1)$-vector with 1 as 
the $i$ th entry and 0 elsewhere. Hence, using $z$ for a $(n+1)$-vector of integration corresponding to $Z$, and the translation invariance of Lebesgue measure,

$$
\begin{aligned}
& \mathbb{E}\left[\exp \left(X_{0}\right) \times \prod_{i=1}^{n}\left(X_{i}^{2}-R_{i i}\right)\right] \\
& =\mathbb{E}\left[\exp \left(e_{0}^{\top} C^{\top} Z\right) \prod_{i=1}^{n}\left(\left|e_{i}^{\top} C^{\top} Z\right|^{2}-R_{i i}\right)\right] \\
& =\frac{1}{(2 \pi)^{(n+1) / 2}} \int \exp \left(e_{0}^{\top} C^{\top} z\right) \prod_{i=1}^{n}\left(\left|e_{i}^{\top} C^{\top} z\right|^{2}-R_{i i}\right) \exp \left(-\frac{1}{2}|z|^{2}\right) \mathrm{d} z \\
& =\frac{1}{(2 \pi)^{(n+1) / 2}} \int \exp \left(e_{0}^{\top} C^{\top}\left(z+C e_{0}\right)\right) \\
& \times \prod_{i=1}^{n}\left(\left|e_{i}^{\top} C^{\top}\left(z+C e_{0}\right)\right|^{2}-R_{i i}\right) \exp \left(-\frac{1}{2}\left|z+C e_{0}\right|^{2}\right) \mathrm{d} z \\
& =\frac{1}{(2 \pi)^{(n+1) / 2}} \int \exp \left(e_{0}^{\top} C^{\top}\left(z+C e_{0}\right)-\frac{1}{2}\left|z+C e_{0}\right|^{2}+\frac{1}{2}|z|^{2}\right) \\
& \times \prod_{i=1}^{n}\left(\left|e_{i}^{\top} C^{\top}\left(z+C e_{0}\right)\right|^{2}-R_{i i}\right) \exp \left(-\frac{1}{2}|z|^{2}\right) \mathrm{d} z \\
& =\frac{1}{(2 \pi)^{(n+1) / 2}} \int \exp \left(\frac{1}{2} e_{0}^{\top} C^{\top} C e_{0}\right) \prod_{i=1}^{n}\left(\left|e_{i}^{\top} C^{\top} z+R_{i 0}\right|^{2}-R_{i i}\right) \exp \left(-\frac{1}{2}|z|^{2}\right) \mathrm{d} z \\
& =\mathbb{E}\left[\exp \left(\frac{1}{2} R_{00}\right) \prod_{i=1}^{n}\left(\left(X_{i}+R_{i 0}\right)^{2}-R_{i i}\right)\right] .
\end{aligned}
$$

Finally, rewrite each factor $\left(\left(X_{i}+R_{0 i}\right)^{2}-R_{i i}\right)$ as $\left(X_{i}^{2}-R_{i i}\right)+2 R_{0 i} X_{i}+R_{0 i}^{2}$. Expanding the product accordingly, we obtain

$$
\begin{aligned}
& \mathbb{E}\left[\exp \left(X_{0}\right) \times \prod_{i=1}^{n}\left(X_{i}^{2}-R_{i i}\right)\right] \\
& \quad=\exp \left(\frac{R_{00}}{2}\right) \sum_{A_{1} \sqcup A_{2} \sqcup A_{3}=\{1,2, \ldots, n\}} 2^{\left|A_{2}\right|} \mathbb{E}\left[\prod_{i \in A_{1}}\left(X_{i}^{2}-R_{i i}\right) \times \prod_{i \in A_{2}} X_{i}\right] \times \prod_{i \in A_{2}} R_{0 i} \times \prod_{i \in A_{3}} R_{0 i}^{2} .
\end{aligned}
$$

The result follows by applying Proposition 12 .

We will also require the following combinatorial lemma in order to separate out groups of integration variables.

LEMMA 14. Suppose that $S=2 \times\{1,2, \ldots, n\} \cup\{n+1, n+2, \ldots, n+2 k\}$ and $p \in$ $\mathcal{P}^{*}(S)$ is a proper pairing. Then it is possible to partition $p$ into three disjoint sets of pairs $p_{1}, p_{2}, p_{3}$ such that the pairs in each $p_{i}(i=1,2,3)$ are pairwise disjoint and $\left\lfloor\frac{n+k}{3}\right\rfloor \leq$ $\left|p_{i}\right| \leq\left\lceil\frac{n+k}{3}\right\rceil$.

Furthermore, if $X=\left(X_{1}, X_{2}, \ldots, X_{n+2 k}\right)$ is a centred multivariate normal random variable, then

$$
\prod_{\Lambda \in p} \mathbb{E}\left[X_{\lambda_{1}} X_{\lambda_{2}}\right]^{2} \leq \frac{1}{3} \prod_{\Lambda \in p_{1}} \mathbb{E}\left[X_{\lambda_{1}} X_{\lambda_{2}}\right]^{6}+\frac{1}{3} \prod_{\Lambda \in p_{2}}\left|\mathbb{E}\left[X_{\lambda_{1}} X_{\lambda_{2}}\right]^{6}+\frac{1}{3} \prod_{\Lambda \in p_{3}}\right| \mathbb{E}\left[X_{\lambda_{1}} X_{\lambda_{2}}\right]^{6} .
$$


PROOF. The pairing $p$ defines a graph on the set of its pairs $\left\{\Lambda_{1}, \ldots, \Lambda_{n+k}\right\}$, where $\Lambda_{i}$ and $\Lambda_{j}$ are connected if and only if $\Lambda_{i} \cap \Lambda_{j} \neq \varnothing$. The maximal degree of this graph is two, hence it is a disjoint union of paths and cycles (and isolated points). Each path or cycle can be coloured with three colours (red, green and blue) so that no neighbouring vertexes are of the same colour and the numbers of vertexes of different colours differ by at most one. Finally colours are interchangeable within each cycle or path, so by careful selection of excess colours for each cycle or path we can ensure that the numbers of vertexes of different colours in the entire graph also differ by at most one.

The colours give us the partition. By definition all the pairs in each $p_{i}$ are disjoint, and by construction $\left\lfloor\frac{n+k}{3}\right\rfloor \leq\left|p_{i}\right| \leq\left\lceil\frac{n+k}{3}\right\rceil$. The bound follows by the inequality between geometric and arithmetic means.

\section{Anomalous scaling for Metropolis-Hastings algorithms in a random environment.}

In this section we develop a framework for proving anomalous scaling results for MetropolisHastings algorithm for product targets which depend on random environments. The aim is to establish sufficient conditions under which the algorithm will exhibit anomalous scaling behaviour for almost all realisations of the random environment. Sections 6 and 7 will then use this framework in the contexts of RWM and MALA algorithms to produce proofs of Theorems 1 and 2.

For the sake of definiteness and computational convenience, we denote the random environment by $B$, and suppose this to be determined by a stationary continuous Gaussian process. A (nonnormalised) random marginal target density is then produced by a map $\xi: \mathbb{R} \times \Omega \rightarrow[0, \infty)$, required to deliver $\int_{\mathbb{R}} \xi(x \mid B) \mathrm{d} x<\infty$ for almost all realisations of the random environment $B$. The normalisation of the random marginal target density is denoted by $\pi: \mathbb{R} \times \Omega \rightarrow \mathbb{R}$, so that

$$
\int_{\mathbb{R}} \pi(x \mid B) \mathrm{d} x=\int_{\mathbb{R}}\left(\frac{\xi(x \mid B)}{\int_{\mathbb{R}} \xi(u \mid B) \mathrm{d} u}\right) \mathrm{d} x=1 .
$$

Finally, let $\rho_{n}(x, y \mid B)=\log \left(\frac{\pi(y \mid B) q_{n}(y, x \mid B)}{\pi(x \mid B) q_{n}(x, y \mid B)}\right)=\log \left(\frac{\xi(y \mid B) q_{n}(y, x \mid B)}{\xi(x \mid B) q_{n}(x, y \mid B)}\right)$ denote the logarithm of the acceptance ratio of the Metropolis-Hastings algorithm with marginal target density $\pi(\cdot \mid B)$ (equivalently $\xi(\cdot \mid B))$ and proposal density $q_{n}: \mathbb{R}^{2} \times \Omega \rightarrow \mathbb{R}$. Note that $q_{n}(x, y \mid B)$ may also depend on the random environment.

In light of Section 3, particularly the central limit Theorem 8, the crucial step is to identify the decay rate of the second moments of the log acceptance ratio $\mathbb{E}_{\pi(\cdot \mid B), q_{n}(\cdot \mid B)}\left[\rho_{n}^{2} \mid B\right]$ or equivalently the decay rate of functionals (differing only by a normalising constant that does not depend on $n$ )

$$
\begin{aligned}
\mathcal{I}_{n}(B) & =\iint_{\mathbb{R}^{2}} \rho_{n}^{2}(x, y \mid B) \xi(x \mid B) q_{n}(x, y \mid B) \mathrm{d} x \mathrm{~d} y \\
& =\iint_{\mathbb{R}^{2}} \rho_{n}^{2}\left(x, x+\sigma_{n} z \mid B\right) \xi(x \mid B) \sigma_{n} q_{n}\left(x, x+\sigma_{n} z \mid B\right) \mathrm{d} x \mathrm{~d} z,
\end{aligned}
$$

for some positive sequence $\sigma_{1}>\sigma_{2}>\sigma_{3}>\cdots>0$.

Throughout the remainder of the paper, for two sequences $a_{1}, a_{2}, \ldots$ and $b_{1}, b_{2}, \ldots$ of positive real numbers, the notation $a_{n} \precsim b_{n}$ indicates that there is a positive constant $C>0$ such that $a_{n} \leq C b_{n}$ holds for all $n$.

We consider the situation in which there is a product target with marginal target density depending on a random environment and a product Metropolis-Hasting proposal. In this section we consider the implications for optimal scaling if the following framework of assumptions is valid. 
ANOMALOUS SCALING FRAMEWORK 15. Let $v_{1}, v_{2}$ be probability density functions on $\mathbb{R}$ with all polynomial moments finite. Fix positive constants $\beta, \gamma$ and $\ell$, and choose a positive integer $m$ such that $m>3+\frac{144 \beta}{\min (24 \gamma, 1)}$. Finally, set $v=v_{1} \times v_{2}$ to be a joint density function, and set $\sigma_{n}=\ell n^{-\frac{1}{2 \beta}}$. The sequence of assumptions (depending implicitly on $\beta, \gamma$, $\ell, m)$ are as follows:

(A) Mixed Gaussian perturbation of log marginal target density:

For every real $x$, the (un-normalised) marginal target density is given by

$$
\xi(x \mid B)=\exp (K(x \mid B)) v_{1}(x),
$$

where $(K(x \mid B): x \in \mathbb{R})$ is a centred Gaussian process such that $K(x \mid B)$ has variance $k(x)$. Furthermore, we suppose $K(x, B)$ has a particular unconditional exponential moment that is finite:

$$
\mathbb{E}\left[\int_{\mathbb{R}} \exp \left(2 m^{2} K(x, B)\right) v_{1}(x) \mathrm{d} x\right]=\int_{\mathbb{R}} \exp \left(2 m^{2} k(x)\right) v_{1}(x) \mathrm{d} x<\infty
$$

(with $m$ chosen as above). Particularly, this moment condition implies $\xi(x \mid B)$ is indeed a target density for almost every realisation of the random environment $B$.

(B) Asymptotic behaviour of perturbation of marginal proposal:

For every real $x, z$ and positive integer $n$, the marginal proposal density $q_{n}$ satisfies

$$
\sigma_{n} q_{n}\left(x, x+\sigma_{n} z \mid B\right)=L_{n}(x, z \mid B) v_{2}(z),
$$

where the random variable $L_{n}(x, z \mid B)$ is controlled by

$$
\iint_{\mathbb{R}^{2}} \mathbb{E}\left[\left|L_{n}(x, z \mid B)-1\right|^{4 m}\right] v(x, z) \mathrm{d} x \mathrm{~d} z \precsim \sigma_{n}^{4 m \gamma} .
$$

(C) Approximate normality of log Metropolis-Hastings ratio (LMHR):

For every real $x, z$ and positive integer $n$

$$
\rho_{n}\left(x, x+\sigma_{n} z \mid B\right)=M_{n}(x, z \mid B)+\Delta_{n}(x, z \mid B),
$$

where (for each $n)$ the random process $\left(M_{n}(x, z \mid B): x, z \in \mathbb{R}\right)$ is a centred Gaussian process such that processes $K$ and $M_{n}$ are also jointly Gaussian. Furthermore, $M_{n}(x, z \mid B)$ has variance $h(x, z) \sigma_{n}^{2 \beta}$, for some function $h$ exhibiting at most polynomial growth, and $\Delta_{n}(x, z \mid B)$ is a random variable satisfying

$$
\iint_{\mathbb{R}^{2}} \mathbb{E}\left[\left|\Delta_{n}(x, z \mid B)\right|^{8 m}\right] v(x, z) \mathrm{d} x \mathrm{~d} z \precsim \sigma_{n}^{8 m \beta+8 m \gamma} .
$$

(D) Asymptotic Weak Dependence: There exist sets $\mathcal{S}_{n} \subset \mathbb{R}^{4}$ taking up increasingly larger parts of the space, specifically $\int_{\mathcal{S}_{n}^{c}} v\left(x_{1}, z_{1}\right) v\left(x_{2}, z_{2}\right) \mathrm{d} x_{1} \mathrm{~d} z_{1} \mathrm{~d} x_{2} \mathrm{~d} z_{2} \precsim \sigma_{n}^{1 / 2}$, and fixed polynomials $g_{1}, g_{2}$, such that for $\left(x_{1}, z_{1}, x_{2}, z_{2}\right) \in \mathcal{S}_{n}$

$$
\left|\mathbb{E}\left[M_{n}\left(x_{1}, z_{1} \mid B\right) M_{n}\left(x_{2}, z_{2} \mid B\right)\right]\right| \leq g_{1}\left(x_{1}, z_{1}, x_{2}, z_{2}\right) \times \sigma_{n}^{2 \beta+\gamma}
$$

while, for all real $x_{1}, z_{1}, x_{2}$,

$$
\left|\mathbb{E}\left[M_{n}\left(x_{1}, z_{1} \mid B\right) K\left(x_{2} \mid B\right)\right]\right| \leq g_{2}\left(x_{1}, z_{1}, x_{2}\right) \times \sigma_{n}^{\beta+\gamma} .
$$

Sections 6 and 7 respectively give concrete examples of anomalous RWM and MALA algorithms in random environment that can be cast in terms of the above framework, that is, they satisfy Assumptions 15.(A)-15.(D). 
Assumptions 15.(A)-15.(D) allow the approximation of functionals $\mathcal{I}_{n}(B)$ by progressively simpler functionals. Initially, consider

$$
\tilde{\mathcal{I}}_{n}(B)=\iint_{\mathbb{R}^{2}} \rho_{n}^{2}\left(x, x+\sigma_{n} z \mid B\right) \exp (K(x \mid B)) v(x, z) \mathrm{d} x \mathrm{~d} z .
$$

We prove a quantitative result which will imply almost sure decay at the same speed as $\mathcal{I}_{n}(B)$.

LEMMA 16. Let Assumptions 15.(A), 15.(B) and 15.(C) be satisfied. Then

$$
\mathbb{E}\left[\left|\mathcal{I}_{n}(B)-\tilde{\mathcal{I}}_{n}(B)\right|^{m}\right] \precsim \sigma_{n}^{2 m \beta+m \gamma} .
$$

PROOF. Writing $v(x, z)=v_{1}(x) v_{2}(z)$ for convenience, the expectation can be rewritten using Assumptions 15.(A) and 15.(B) of Framework 15 and then bounded by a combination of Jensen's inequality and double usage of Cauchy-Schwarz inequality (all with respect to $v(x, z) \mathrm{d} x \mathrm{~d} z \mathrm{~d} \mathbb{P})$ to give

$$
\begin{aligned}
& \mathbb{E}\left[\left|\mathcal{I}_{n}(B)-\tilde{\mathcal{I}}_{n}(B)\right|^{m}\right] \\
& \leq \mathbb{E}\left[\iint_{\mathbb{R}^{2}} \rho_{n}^{2 m}\left(x, x+\sigma_{n} z \mid B\right) \exp (m K(x \mid B))\left|L_{n}(x, z \mid B)-1\right|^{m} v(x, z) \mathrm{d} x \mathrm{~d} z\right] \\
& \leq\left(\iint_{\mathbb{R}^{2}} \mathbb{E}[\exp (2 m K(x \mid B))] v(x, z) \mathrm{d} x \mathrm{~d} z\right)^{1 / 2} \\
& \quad \times\left(\iint_{\mathbb{R}^{2}} \mathbb{E}\left[\left(L_{n}(x, z \mid B)-1\right)^{4 m}\right] v(x, z) \mathrm{d} x \mathrm{~d} z\right)^{1 / 4} \\
& \quad \times\left(\iint_{\mathbb{R}^{2}} \mathbb{E}\left[\rho_{n}^{8 m}\left(x, x+\sigma_{n} z \mid B\right)\right] v(x, z) \mathrm{d} x \mathrm{~d} z\right)^{1 / 4} .
\end{aligned}
$$

The first factor is bounded by application of Assumption 15.(A) followed by marginalization over $z$. The second factor decays at least as $\sigma_{n}^{m \gamma}$ by Assumption 15.(B).

The proof will be concluded once we establish the last factor decays at least as $\sigma_{n}^{2 m \beta}$. Indeed

$$
\begin{aligned}
& \left(\iint_{\mathbb{R}^{2}} \mathbb{E}\left[\rho_{n}^{8 m}\left(x, x+\sigma_{n} z \mid B\right)\right] v(x, z) \mathrm{d} x \mathrm{~d} z\right)^{1 / 4} \\
& =\left(\mathbb{E}\left[\iint_{\mathbb{R}^{2}}\left(M_{n}(x, z \mid B)+\Delta_{n}(x, z \mid B)\right)^{8 m} v(x, z) \mathrm{d} x \mathrm{~d} z\right]\right)^{1 / 4} \\
& \quad \leq 2^{2 m-1} \cdot\left(\left(\mathbb{E}\left[\iint_{\mathbb{R}^{2}} M_{n}(x, z \mid B)^{8 m} v(x, z) \mathrm{d} x \mathrm{~d} z\right]\right)^{1 / 4}\right. \\
& \left.\quad+\left(\mathbb{E}\left[\iint_{\mathbb{R}^{2}} \Delta_{n}(x, z \mid B)^{8 m} v(x, z) \mathrm{d} x \mathrm{~d} z\right]\right)^{1 / 4}\right) \\
& \quad \leq 2^{2 m-1} \cdot\left(\left(\mathbb{E}\left[N(0,1)^{8 m}\right] \iint_{\mathbb{R}^{2}} h(x, z)^{4 m} v(x, z) \mathrm{d} x \mathrm{~d} z\right)^{1 / 4} \sigma_{n}^{2 m \beta}+C \sigma_{n}^{2 m \beta+2 m \gamma}\right) \\
& \precsim \sigma_{n}^{2 m \beta},
\end{aligned}
$$

where $C$ is some positive constant. The identity holds by Assumption 15.(C). The first inequality follows from the elementary bound $(a+b)^{2 m} \leq 2^{2 m-1}\left(a^{2 m}+b^{2 m}\right)$ together with application of a triangle inequality in $L^{4}(v \times \mathbb{P})$ norm. The remainder follows from the Fubini-Tonelli theorem and the details of Assumption 15.(C). 
The functionals $\tilde{I}_{n}(B)$ can now be simplified further by approximating $\rho_{n}\left(x, x+\sigma_{n} z \mid B\right) \approx$ $M_{n}(x, z \mid B)$, and controlling the approximation using Assumptions 15.(A) and 15.(C). Let

$$
\hat{\mathcal{I}}_{n}(B)=\iint_{\mathbb{R}^{2}} M_{n}^{2}(x, z \mid B) \exp (K(x \mid B)) v(x, z) \mathrm{d} x \mathrm{~d} z .
$$

Again the functionals $\tilde{\mathcal{I}}_{n}(B)$ and $\hat{\mathcal{I}}_{n}(B)$ can be shown to be close to each other.

LEMma 17. Suppose Assumptions 15.(A) and 15.(C) are satisfied. Then

$$
\mathbb{E}\left[\left|\tilde{\mathcal{I}}_{n}(B)-\hat{\mathcal{I}}_{n}(B)\right|^{m}\right] \precsim \sigma_{n}^{2 m \beta+m \gamma} \text {. }
$$

PROOF. Arguing as in Lemma 16, Jensen's inequality yields

$$
\left|\tilde{\mathcal{I}}_{n}(B)-\hat{\mathcal{I}}_{n}(B)\right|^{m} \leq \iint_{\mathbb{R}^{2}}\left|\rho_{n}^{2}\left(x, x+\sigma_{n} z \mid B\right)-M_{n}^{2}(x, z \mid B)\right|^{m} \exp (m K(x \mid B)) v(x, z) \mathrm{d} x \mathrm{~d} z .
$$

Recall that by Assumption 15.(C)

$$
\rho_{n}^{2}\left(x, x+\sigma_{n} z \mid B\right)-M_{n}^{2}(x, z \mid B)=\Delta_{n}(x, z \mid B)\left(2 M_{n}^{2}(x, z \mid B)+\Delta_{n}(x, z \mid B)\right) .
$$

Exchanging the expectation with the double integral using the Fubini-Tonelli theorem, and then applying the Cauchy-Schwarz inequality twice over,

$$
\begin{aligned}
\mathbb{E}\left[\left|\tilde{\mathcal{I}}_{n}(B)-\hat{\mathcal{I}}_{n}(B)\right|^{m}\right] \leq & \left.\left(\iint_{\mathbb{R}^{2}} \mathbb{E}[\exp (2 m K(x \mid B))] v(x, z) \mathrm{d} x \mathrm{~d} z\right)\right)^{1 / 2} \\
& \times\left(\iint_{\mathbb{R}^{2}} \mathbb{E}\left[\Delta_{n}(x, z \mid B)^{4 m}\right] v(x, z) \mathrm{d} x \mathrm{~d} z\right)^{1 / 4} \\
& \times\left(\iint_{\mathbb{R}^{2}} \mathbb{E}\left[\left(2 M_{n}(x, z \mid B)+\Delta_{n}(x, z \mid B)\right)^{4 m}\right] v(x, z) \mathrm{d} x \mathrm{~d} z\right)^{1 / 4} .
\end{aligned}
$$

As in the proof of Lemma 16, Assumption 15.(A) implies the first factor is bounded and Assumption 15.(C) guarantees second factor decays at least as $\sigma_{n}^{m \beta+m \gamma}$ and the third as $\sigma_{n}^{m \beta}$.

The final step is to consider the functional obtained from $\hat{\mathcal{I}}_{n}(B)$ by replacing $\left(M_{n}(x\right.$, $z \mid B))^{2}$ by its expectation (see Assumptions 15.(A) and 15.(C)):

$$
\begin{aligned}
\mathcal{J}_{n}(B) & =\iint_{\mathbb{R}^{2}} \mathbb{E}\left[\left(M_{n}(x, z \mid B)\right)^{2}\right] \exp (K(x \mid B)) v(x, z) \mathrm{d} x \mathrm{~d} z . \\
& =\sigma_{n}^{2 \beta} \cdot \iint_{\mathbb{R}^{2}} h(x, z) \xi(x \mid B) v_{2}(z) \mathrm{d} x \mathrm{~d} z \\
& =\sigma_{n}^{2 \beta} \cdot \iint_{\mathbb{R}^{2}} h(x, z) \exp (K(x \mid B)) v_{1}(x) v_{2}(z) \mathrm{d} x \mathrm{~d} z .
\end{aligned}
$$

Note that the double integral is almost surely finite: this follows from the polynomial growth of $h(x, z)$ (Assumption 15.(C)), Cauchy-Schwarz inequality, the fact that the densities $v_{1}$ and $v_{2}$ have finite polynomial moments (stipulated in the Framework 15), and the fact that $\exp (k(x))$ is integrable with respect to $v_{1}$ (Assumption 15.(A)).

Again we need to establish that the functionals $\hat{\mathcal{I}}_{n}(B)$ and $\mathcal{J}_{n}(B)$ are close.

LEMmA 18. Let Assumptions 15.(A), 15.(C) and 15.(D) be satisfied. Then

$$
\mathbb{E}\left[\left|\hat{\mathcal{I}}_{n}(B)-\mathcal{J}_{n}(B)\right|^{m}\right] \precsim \sigma_{n}^{2 m \beta+m \min \left(\frac{\gamma}{2}, \frac{1}{48}\right)} .
$$


ProOF. It suffices to bound $\mathbb{E}\left[\left|\hat{\mathcal{I}}_{n}(B)-\mathcal{J}_{n}(B)\right|^{2 m}\right]$, since by Jensen's inequality

$$
\mathbb{E}\left[\left|\hat{\mathcal{I}}_{n}(B)-\mathcal{J}_{n}(B)\right|^{m}\right] \leq \mathbb{E}\left[\left|\hat{\mathcal{I}}_{n}(B)-\mathcal{J}_{n}(B)\right|^{2 m}\right]^{1 / 2} .
$$

Formulae (12) for $\hat{\mathcal{I}}_{n}(B)$ and (13) for $\mathcal{J}_{n}(B)$ together imply

$$
\hat{\mathcal{I}}_{n}(B)-\mathcal{J}_{n}(B)=\iint_{\mathbb{R}^{2}}\left(M_{n}(x, z \mid B)^{2}-\mathbb{E}\left[M_{n}(x, z \mid B)^{2}\right]\right) \exp (K(x \mid B)) v(x, z) \mathrm{d} x \mathrm{~d} z
$$

and consequently

$$
\begin{aligned}
& \left(\hat{\mathcal{I}}_{n}(B)-\mathcal{J}_{n}(B)\right)^{2 m} \\
& =\int \cdots \int_{\mathbb{R}^{4 m}}\left(\prod_{i=1}^{2 m}\left(M_{n}\left(x_{i}, z_{i} \mid B\right)^{2}-\mathbb{E}\left[M_{n}\left(x_{i}, z_{i} \mid B\right)^{2}\right]\right)\right) \times \exp \left(\sum_{i=1}^{2 m}\left(K\left(x_{i} \mid B\right)\right)\right) \\
& \quad \times \prod_{i=1}^{2 m}\left(v\left(x_{i}, z_{i}\right) \mathrm{d} x_{i} \mathrm{~d} z_{i}\right) \\
& =\int \cdots \int_{\mathbb{R}^{4 m}}\left(\prod_{i=1}^{2 m}\left(M_{i}^{2}-R_{i i}\right)\right) \times \exp (\bar{K}) \times\left(\prod_{i=1}^{2 m} v\left(x_{i}, z_{i}\right) \mathrm{d} x_{i} \mathrm{~d} z_{i}\right),
\end{aligned}
$$

where we abbreviate notation by writing $M_{i}=M_{n}\left(x_{i}, z_{i} \mid B\right), R_{i i}=\mathbb{E}\left[M_{i}^{2}\right]$ and $\bar{K}=$ $\sum_{i=1}^{2 m}\left(K\left(x_{i} \mid B\right)\right)$. Note that the various $M_{i}$ and $K\left(x_{j} \mid B\right)$ are not necessarily independent, and typically will not be so.

Using the Fubini-Tonelli theorem to exchange the expectation in $\mathbb{E}\left[\left(\hat{\mathcal{I}}_{n}(B)-\mathcal{J}_{n}(B)\right)^{2 m}\right]$ with the implicit multiple integrals, we now obtain

$$
\mathbb{E}\left[\left(\hat{\mathcal{I}}_{n}(B)-\mathcal{J}_{n}(B)\right)^{2 m}\right]=\int \cdots \int_{\mathbb{R}^{4 m}} \mathbb{E}\left[\exp (\bar{K}) \prod_{i=1}^{2 m}\left(M_{i}^{2}-R_{i i}\right)\right] \prod_{i=1}^{2 m}\left(v\left(x_{i}, z_{i}\right) \mathrm{d} x_{i} \mathrm{~d} z_{i}\right)
$$

By Lemma 13 the expectation $\mathbb{E}\left[\exp (\bar{K}) \prod_{i=1}^{2 m}\left(M_{i}^{2}-R_{i i}\right)\right]$ equals

$$
\begin{aligned}
& \exp \left(\frac{1}{2} \mathbb{E}\left[\bar{K}^{2}\right]\right) \sum_{\substack{A_{1} \cup A_{2} \cup A_{3}=\{1,2, \ldots, 2 m\} \\
A_{1} \cap A_{2}=A_{1} \cap A_{3}=A_{2} \cap A_{3}=\varnothing \\
\left|A_{2}\right| \text { is even }}} \sum_{p \in \mathcal{P}^{*}\left(2 A_{1} \cup A_{2}\right)} 2^{\left|A_{2}\right|} \prod_{i \in A_{2}} \mathbb{E}\left[M_{i} \bar{K}\right] \\
& \times \prod_{i \in A_{3}} \mathbb{E}\left[M_{i} \bar{K}\right]^{2} \times \prod_{\Lambda \in p} \mathbb{E}\left[M_{\lambda_{1}} M_{\lambda_{2}}\right] .
\end{aligned}
$$

Inserting the above into (14), we obtain

$$
\begin{aligned}
\mathbb{E}\left[\left(\hat{\mathcal{I}}_{n}(B)-\mathcal{J}_{n}(B)\right)^{2 m}\right]= & \sum_{\substack{A_{1} \cup A_{2} \cup A_{3}=\{1,2, \ldots, 2 m\} \\
A_{1} \cap A_{2}=A_{1} \cap A_{3}=A_{2} \cap A_{3}=\varnothing \\
\left|A_{2}\right| \text { is even }}} \sum_{p \in \mathcal{P}^{*}\left(2 A_{1} \cup A_{2}\right)} 2^{\left|A_{2}\right|} \\
& \times \int \cdots \int_{\mathbb{R}^{4 m}} \prod_{i \in A_{2}} \mathbb{E}\left[M_{i} \bar{K}\right] \cdot \prod_{i \in A_{3}} \mathbb{E}\left[M_{i} \bar{K}\right]^{2} \cdot \prod_{\Lambda \in p} \mathbb{E}\left[M_{\lambda_{1}} M_{\lambda_{2}}\right] \\
& \times \exp \left(\mathbb{E}\left[\frac{1}{2} \bar{K}^{2}\right]\right) \cdot \prod_{i=1}^{2 m}\left(v\left(x_{i}, z_{i}\right) \mathrm{d} x_{i} \mathrm{~d} z_{i}\right) .
\end{aligned}
$$

Now focus attention on a typical summand in the above sum. This corresponds to fixing a partition $A_{1}, A_{2}, A_{3}$ with prescribed properties and a proper pairing $p$ of $2 A_{1} \cup A_{2}$. Applying 
the Cauchy-Schwarz inequality with respect to the measure $\prod_{i=1}^{2 m}\left(v\left(x_{i}, z_{i}\right) \mathrm{d} x_{i} \mathrm{~d} z_{i}\right)$,

$$
\begin{aligned}
\int \cdots & \int_{\mathbb{R}^{4 m}} \prod_{i \in A_{2}} \mathbb{E}\left[M_{i} \bar{K}\right] \cdot \prod_{i \in A_{3}} \mathbb{E}\left[M_{i} \bar{K}\right]^{2} \cdot \prod_{\Lambda \in p} \mathbb{E}\left[M_{\lambda_{1}} M_{\lambda_{2}}\right] \\
& \times \exp \left(\frac{1}{2} \mathbb{E}\left[\bar{K}^{2}\right]\right) \cdot \prod_{i=1}^{2 m}\left(v\left(x_{i}, z_{i}\right) \mathrm{d} x_{i} \mathrm{~d} z_{i}\right) \\
\leq & \left(\int \cdots \int_{\mathbb{R}^{4 m}} \prod_{i \in A_{2}} \mathbb{E}\left[M_{i} \bar{K}\right]^{2} \cdot \prod_{i \in A_{3}} \mathbb{E}\left[M_{i} \bar{K}\right]^{4} \cdot \exp \left(\mathbb{E}\left[\bar{K}^{2}\right]\right) \cdot \prod_{i=1}^{2 m}\left(v\left(x_{i}, z_{i}\right) \mathrm{d} x_{i} \mathrm{~d} z_{i}\right)\right)^{1 / 2} \\
& \times\left(\int \cdots \int_{\mathbb{R}^{4 m}} \prod_{\Lambda \in p} \mathbb{E}\left[M_{\lambda_{1}} M_{\lambda_{2}}\right]^{2} \cdot \prod_{i=1}^{2 m}\left(v\left(x_{i}, z_{i}\right) \mathrm{d} x_{i} \mathrm{~d} z_{i}\right)\right)^{1 / 2} \cdot
\end{aligned}
$$

Consider the first factor. We can bound each $\left|\mathbb{E}\left[M_{i} \bar{K}\right]\right|$ by $\left|\mathbb{E}\left[M_{i} \bar{K}\right]\right| \leq g\left(z_{i}, x_{1}, \ldots\right.$, $\left.x_{n}\right) \sigma_{n}^{\beta+\gamma}$, using a polynomial $g\left(z_{i}, x_{1}, \ldots, x_{n}\right)=\sum_{j=1}^{2 m} g_{2}\left(x_{i}, z_{i}, x_{j}\right)$, generated from the second point of Assumption 15.(D).

By the Cauchy-Schwarz inequality,

$$
\left(\frac{1}{2 m}\right)^{2} \bar{K}^{2}=\left(\frac{1}{2 m} \sum_{i=1}^{2 m} K\left(x_{i} \mid B\right)\right)^{2} \leq \frac{1}{2 m} \sum_{i=1}^{2 m} K\left(x_{i} \mid B\right)^{2}
$$

and so

$$
\begin{aligned}
& \prod_{i \in A_{2}} \mathbb{E}\left[M_{i} \bar{K}\right]^{2} \cdot \prod_{i \in A_{3}} \mathbb{E}\left[M_{i} \bar{K}\right]^{4} \cdot \exp \left(\mathbb{E}\left[\bar{K}^{2}\right]\right) \\
& \quad \leq \prod_{i \in A_{2}} \mathbb{E}\left[M_{i} \bar{K}\right]^{2} \cdot \prod_{i \in A_{3}} \mathbb{E}\left[M_{i} \bar{K}\right]^{4} \cdot \exp \left(2 m \sum_{j=1}^{2 m} \mathbb{E}\left[K\left(x_{j} \mid B\right)^{2}\right]\right) .
\end{aligned}
$$

Hence, Assumptions 15.(A) and 15.(D) yield

$$
\begin{aligned}
& \prod_{i \in A_{2}} \mathbb{E}\left[M_{i} \bar{K}\right]^{2} \cdot \prod_{i \in A_{3}} \mathbb{E}\left[M_{i} \bar{K}\right]^{4} \cdot \exp \left(\mathbb{E}\left[\bar{K}^{2}\right]\right) \\
& \leq \exp \left(2 m \sum_{j=1}^{2 m} k\left(x_{j}\right)\right) \prod_{i \in A_{2}} g\left(z_{i}, x_{1}, \ldots, x_{n}\right)^{2} \prod_{i \in A_{3}} g\left(z_{i}, x_{1}, \ldots, x_{n}\right)^{4} \\
& \quad \times \sigma_{n}^{\left(2\left|A_{2}\right|+4\left|A_{3}\right|\right)(\beta+\gamma)}
\end{aligned}
$$

Application of the Cauchy-Schwarz inequality, and the exponential integrability of $2 m^{2} k(x)$ (with respect to $v_{1}(x) \mathrm{d} x$ ) assured by Assumption 15.(A), shows that this is integrable with respect to the probability measure $\prod_{i=1}^{2 m}\left(v\left(x_{i}, z_{i}\right) \mathrm{d} x_{i} \mathrm{~d} z_{i}\right)$. Consequently we obtain

$$
\begin{aligned}
& \left(\int \cdots \int_{\mathbb{R}^{4 m}} \prod_{i \in A_{2}} \mathbb{E}\left[M_{i} \bar{K}\right]^{2} \cdot \prod_{i \in A_{3}} \mathbb{E}\left[M_{i} \bar{K}\right]^{4} \cdot \exp \left(\mathbb{E}\left[\bar{K}^{2}\right]\right) \cdot \prod_{i=1}^{2 m} v\left(x_{i}, z_{i}\right) \mathrm{d} x_{i} \mathrm{~d} z_{i}\right)^{1 / 2} \\
& \quad \precsim \sigma_{n}^{\left(\left|A_{2}\right|+2\left|A_{3}\right|\right)(\beta+\gamma)} .
\end{aligned}
$$

Consider now the second factor in (16). As $p$ is a proper pairing, Lemma 14 asserts there is a partition of $p$ into three sets of pairs $p_{1}, p_{2}, p_{3}$ of size at least $\left\lfloor\left|A_{1}\right| / 3+\left|A_{2}\right| / 6\right\rfloor$ so that all pairs within each $p_{i}$ are disjoint and moreover

$$
\prod_{\Lambda \in p} \mathbb{E}\left[M_{\lambda_{1}} M_{\lambda_{2}}\right]^{2} \leq \frac{1}{3} \prod_{\Lambda \in p_{1}} \mathbb{E}\left[M_{\lambda_{1}} M_{\lambda_{2}}\right]^{6}+\frac{1}{3} \prod_{\Lambda \in p_{2}} \mathbb{E}\left[M_{\lambda_{1}} M_{\lambda_{2}}\right]^{6}+\frac{1}{3} \prod_{\Lambda \in p_{3}} \mathbb{E}\left[M_{\lambda_{1}} M_{\lambda_{2}}\right]^{6}
$$


This allows us to split the integral over $\mathbb{R}^{4 m}$ into a product of integrals over $\mathbb{R}^{4}$

$$
\begin{aligned}
& \int \cdots \int_{\mathbb{R}^{4 m}} \prod_{\Lambda \in p} \mathbb{E}\left[M_{\lambda_{1}} M_{\lambda_{2}}\right]^{2} \cdot \prod_{i=1}^{2 m} v\left(x_{i}, z_{i}\right) \mathrm{d} x_{i} \mathrm{~d} z_{i} \\
& \quad \leq \frac{1}{3} \sum_{j=1}^{3} \int \cdots \int_{\mathbb{R}^{4 m}} \prod_{\Lambda \in p_{j}} \mathbb{E}\left[M_{\lambda_{1}} M_{\lambda_{2}}\right]^{6} \prod_{i=1}^{2 m} v\left(x_{i}, z_{i}\right) \mathrm{d} x_{i} \mathrm{~d} z_{i} \\
& \quad=\frac{1}{3} \sum_{j=1}^{3} \prod_{\Lambda \in p_{j}} \int_{\mathbb{R}^{4}} \mathbb{E}\left[M_{\lambda_{1}} M_{\lambda_{2}}\right]^{6} v\left(x_{\lambda_{1}}, z_{\lambda_{1}}\right) v\left(x_{\lambda_{2}}, z_{\lambda_{2}}\right) \mathrm{d} x_{\lambda_{1}} \mathrm{~d} z_{\lambda_{1}} \mathrm{~d} x_{\lambda_{2}} \mathrm{~d} z_{\lambda_{2}} .
\end{aligned}
$$

The last equality holds because pairs within each $p_{j}$ are by construction disjoint which imposes a product structure on the high-dimensional integral.

For each of the factors of (19), the first bound of Assumption 15.(D) yields

$$
\int_{\mathcal{S}_{n}} \mathbb{E}\left[M_{\lambda_{1}} M_{\lambda_{2}}\right]^{6} v\left(x_{\lambda_{1}}, z_{\lambda_{1}}\right) v\left(x_{\lambda_{2}}, z_{\lambda_{2}}\right) \mathrm{d} x_{\lambda_{1}} \mathrm{~d} z_{\lambda_{1}} \mathrm{~d} x_{\lambda_{2}} \mathrm{~d} z_{\lambda_{2}} \precsim \sigma_{n}^{12 \beta+6 \gamma} .
$$

The Cauchy-Schwarz inequality, together with Assumptions 15.(C) and 15.(D) control the integral of the set $\mathcal{S}_{n}$,

$$
\begin{aligned}
& \int_{\mathcal{S}_{n}^{c}} \mathbb{E}\left[M_{\lambda_{1}} M_{\lambda_{2}}\right]^{6} v\left(x_{\lambda_{1}}, z_{\lambda_{1}}\right) v\left(x_{\lambda_{2}}, z_{\lambda_{2}}\right) \mathrm{d} x_{\lambda_{1}} \mathrm{~d} z_{\lambda_{1}} \mathrm{~d} x_{\lambda_{2}} \mathrm{~d} z_{\lambda_{2}} \\
& \quad \leq \int_{\mathbb{R}^{4}} \mathbb{E}\left[M_{\lambda_{1}}^{2}\right]^{3} \mathbb{E}\left[M_{\lambda_{2}}^{2}\right]^{3} 1_{\mathcal{S}_{n}^{c}}\left(x_{\lambda_{1}}, z_{\lambda_{1}}, x_{\lambda_{2}}, z_{\lambda_{2}}\right) v\left(x_{\lambda_{1}}, z_{\lambda_{1}}\right) v\left(x_{\lambda_{2}}, z_{\lambda_{2}}\right) \mathrm{d} x_{\lambda_{1}} \mathrm{~d} z_{\lambda_{1}} \mathrm{~d} x_{\lambda_{2}} \mathrm{~d} z_{\lambda_{2}} \\
& \quad \precsim \sigma_{n}^{12 \beta}\left(\int_{\mathcal{S}_{n}^{c}} v\left(x_{\lambda_{1}}, z_{\lambda_{1}}\right) v\left(x_{\lambda_{2}}, z_{\lambda_{2}}\right) \mathrm{d} x_{\lambda_{1}} \mathrm{~d} z_{\lambda_{1}} \mathrm{~d} x_{\lambda_{2}} \mathrm{~d} z_{\lambda_{2}}\right)^{1 / 2} \precsim \sigma_{n}^{12 \beta+\frac{1}{4}} .
\end{aligned}
$$

Together the above bounds give

$$
\int_{\mathbb{R}^{4}} \mathbb{E}\left[M_{\lambda_{1}} M_{\lambda_{2}}\right]^{6} v\left(x_{\lambda_{1}}, z_{\lambda_{1}}\right) v\left(x_{\lambda_{2}}, z_{\lambda_{2}}\right) \mathrm{d} x_{\lambda_{1}} \mathrm{~d} z_{\lambda_{1}} \mathrm{~d} x_{\lambda_{2}} \mathrm{~d} z_{\lambda_{2}} \precsim \sigma_{n}^{12 \beta+6 \min \left(\gamma, \frac{1}{24}\right)} .
$$

Since Lemma 14 asserts that each set of pairs $p_{j}$ contains at least $\left\lfloor\left|A_{1}\right| / 3+\left|A_{2}\right| / 6\right\rfloor$ pairs, the above together with (19) gives

$$
\begin{aligned}
& \left(\int \cdots \int_{\mathbb{R}^{4 m}} \prod_{\Lambda \in p} \mathbb{E}\left[M_{\lambda_{1}} M_{\lambda_{2}}\right]^{2} \cdot \prod_{i=1}^{2 m} v\left(x_{i}, z_{i}\right) \mathrm{d} x_{i} \mathrm{~d} z_{i}\right)^{1 / 2} \\
& \quad \precsim \sigma_{n}^{\left(6 \beta+3 \min \left(\gamma, \frac{1}{24}\right)\right)\left\lfloor\left|A_{1}\right| / 3+\left|A_{2}\right| / 6\right\rfloor} .
\end{aligned}
$$

Combining (20) with (16) and (18), we obtain the following bound for each fixed partition:

$$
\begin{aligned}
& \mid \int \cdots \int_{\mathbb{R}^{4 m}} \prod_{i \in A_{2}} \mathbb{E}\left[M_{i} \bar{K}\right] \cdot \prod_{i \in A_{3}} \mathbb{E}\left[M_{i} \bar{K}\right]^{2} \cdot \prod_{\Lambda \in p} \mathbb{E}\left[M_{\lambda_{1}} M_{\lambda_{2}}\right] \\
& \times \exp \left(\mathbb{E}\left[\frac{1}{2} \bar{K}^{2}\right]\right) \cdot \prod_{i=1}^{2 m} v\left(x_{i}, z_{i}\right) \mathrm{d} x_{i} \mathrm{~d} z_{i} \mid \\
& \precsim \sigma_{n}^{\left(\left|A_{2}\right|+2\left|A_{3}\right|\right)(\beta+\gamma)} \times \sigma_{n}^{\left\lfloor\left|A_{1}\right| / 3+\left|A_{2}\right| / 6\right\rfloor \times\left(6 \beta+3 \min \left(\gamma, \frac{1}{24}\right)\right)} \text {. } \\
& \precsim \sigma_{n}^{2 \beta\left(\left|A_{1}\right|+\left|A_{2}\right|+\left|A_{3}\right|\right)} \times \sigma_{n}^{2 \gamma\left|A_{3}\right|+\left(\gamma+\frac{1}{2} \min \left(\gamma, \frac{1}{24}\right)\right)\left|A_{2}\right|+\min \left(\gamma, \frac{1}{24}\right)\left|A_{1}\right|-6 \beta-3 \min \left(\gamma, \frac{1}{24}\right)} \\
& \leq \sigma_{n}^{4 m \beta} \times \sigma_{n}^{2 m \min \left(\gamma, \frac{1}{24}\right)} \times \sigma_{n}^{-6 \beta-3 \min \left(\gamma, \frac{1}{24}\right)} \leq \sigma_{n}^{4 m \beta} \times \sigma_{n}^{m \min \left(\gamma, \frac{1}{24}\right)} .
\end{aligned}
$$


The argument for this uses $\left|A_{1}\right|+\left|A_{2}\right|+\left|A_{3}\right|=2 m$ together with crude bounds to reduce coefficients of remaining $A_{1}, A_{2}, A_{3}$ to $\min \left(\gamma, \frac{1}{24}\right)$ and then employs $m>3+\frac{144 \beta}{\min (24 \gamma, 1)}$ as stipulated in the Framework 15.

The above bound no longer depends on the choice of partition $A_{1}, A_{2}, A_{3}$ and so can be used in (15) to achieve a bound of

$$
\left(\hat{\mathcal{I}}_{n}(B)-\mathcal{J}_{n}(B)\right)^{2 m} \leq \text { constant } \times \sigma_{n}^{4 m \beta+m \min \left(\gamma, \frac{1}{24}\right)},
$$

where the constant depends on $m$ but not on $n$. As noted at the start of the proof, this establishes the lemma.

We now require the following application of the Borel-Cantelli lemma.

Proposition 19. Let $U_{1}, U_{2}, \ldots$ and $\tilde{U}_{1}, \tilde{U}_{2}, \ldots$ be sequences of random variables, let $\delta_{1}, \delta_{2}, \ldots$ be a positive sequence converging to zero, and suppose $\kappa$ is a positive constant. Assume there is a constant $C>0$ and an integer $m>\frac{1}{\kappa}$ such that the inequality $\mathbb{E}\left[\mid U_{n}-\right.$ $\left.\left.\tilde{U}_{n}\right|^{m}\right] \leq C \delta_{n}^{m} n^{-m \kappa}$ is satisfied for every $n$. Then $\mathbb{P}\left[\delta_{n}^{-1}\left(U_{n}-\tilde{U}_{n}\right) \stackrel{n \rightarrow \infty}{\longrightarrow} 0\right]=1$.

PROOF. Take an arbitrary $\epsilon>0$. By Markov's inequality,

$$
\mathbb{P}\left[\left|U_{n}-\tilde{U}_{n}\right|>\epsilon \delta_{n}\right] \leq \frac{1}{\epsilon^{m} \delta_{n}^{m}} \mathbb{E}\left[\left|U_{n}-\tilde{U}_{n}\right|^{m}\right] \leq \frac{C}{\epsilon^{m}} n^{-m \kappa} .
$$

Summing over $n=1,2, \ldots$, and noting that $m \kappa>1$,

$$
\sum_{n=1}^{\infty} \mathbb{P}\left[\left|U_{n}-\tilde{U}_{n}\right|>\epsilon \delta_{n}\right] \leq \frac{C}{\epsilon^{m}} \sum_{n=1}^{\infty} n^{-m \kappa}<\infty .
$$

It now follows from the Borel-Cantelli lemma that $\mathbb{P}\left[\left|U_{n}-\tilde{U}_{n}\right|>\epsilon \delta_{n}\right.$ i.o. $]=0$. Since $\epsilon>0$ was arbitrary, the result follows.

This enables us to show that the functionals $\mathcal{I}_{n}(B), \tilde{\mathcal{I}}_{n}(B), \hat{\mathcal{I}}_{n}(B)$ and $\mathcal{J}_{n}(B)$ indeed decay with the same speed almost surely (for almost all realisations of the random environment $B$ ) and thus identify the almost sure decay of $\mathcal{I}_{n}(B)$.

PROPOSITION 20. Let the assumptions of Framework 15 be satisfied. Then (almost surely in the random environment $B$ )

$$
\left.\sigma_{n}^{-2 \beta} \mathcal{I}_{n}(B) \stackrel{n \rightarrow \infty}{\longrightarrow} \iint_{\mathbb{R}^{2}} h(x, z) \xi(x \mid B)\right) v_{2}(z) \mathrm{d} x \mathrm{~d} z
$$

So in this case $\mathcal{I}_{n}(B)$ almost surely decays as $\sigma_{n}^{2 \beta}$.

PROOF. Note that the Framework 15 includes a stipulation that $\sigma_{n}=\ell n^{-\frac{1}{2 \beta}}$, as well as a requirement that $m>3+\frac{144 \beta}{\min (24 \gamma, 1)}$.

Apply Proposition 19 together with Lemma 16 in the case that $U_{n}=\mathcal{I}_{n}, \tilde{U}_{n}=\tilde{\mathcal{I}}_{n}, \delta_{n}=\sigma_{n}^{2 \beta}$ and $\kappa=\frac{\gamma}{2 \beta}$. Since $m \kappa=m \frac{\gamma}{2 \beta} \geq 3>1$, it follows that the difference $\left|\mathcal{I}_{n}(B)-\tilde{\mathcal{I}}_{n}(B)\right|$ almost surely decays faster than $\sigma_{n}^{2 \beta}$.

Similarly, apply Proposition 19 together with Lemma 17 in the case that $U_{n}=\tilde{\mathcal{I}}_{n}, \tilde{U}_{n}=$ $\hat{\mathcal{I}}_{n}, \delta_{n}=\sigma_{n}^{2 \beta}$ and $\kappa=\frac{\gamma}{2 \beta}$. Since again $m \kappa=m \frac{\gamma}{2 \beta} \geq 3>1$, it follows that the difference $\left|\tilde{\mathcal{I}}_{n}(B)-\hat{\mathcal{I}}_{n}(B)\right|$ almost surely decays faster than $\sigma_{n}^{2 \beta}$. 
Finally, apply Proposition 19 together with Lemma 18 in the case that $U_{n}=\hat{\mathcal{I}}_{n}, \tilde{U}_{n}=\mathcal{J}_{n}$, $\delta_{n}=\sigma_{n}^{2 \beta}$ and $\kappa=\frac{\min (24 \gamma, 1)}{96 \beta}$. Now $m \kappa=m \frac{\min (24 \gamma, 1)}{96 \beta}>\frac{144 \beta}{\min (24 \gamma, 1)} \frac{\min (24 \gamma, 1)}{96 \beta}=\frac{3}{2}>1$, and so the difference $\left|\hat{\mathcal{I}}_{n}(B)-\mathcal{J}_{n}(B)\right|$ almost surely decays faster than $\sigma_{n}^{2 \beta}$.

Consequently the difference $\left|I_{n}(B)-\mathcal{J}_{n}(B)\right|$ almost surely decays faster than $\sigma_{n}^{2 \beta}$. But $\mathcal{J}_{n}(B)$ is calculated exactly in (13), and demonstrably almost surely decays exactly as $\sigma_{n}^{2 \beta}$. Consequently the same must hold for $I_{n}(B)$ and so the proposition follows.

Since the random targets $\xi(\cdot \mid B)$ ) are almost surely integrable and independent of $n$ (Assumption 15.(A)), the following corollary follows by normalisation.

COROLlARY 21. Let the assumptions of Framework 15 be satisfied. Then (almost surely in the random environment $B$ )

$$
\sigma_{n}^{-2 \beta} \mathbb{E}_{\pi(\cdot \mid B), q_{n}(\cdot \mid B)}\left[\rho_{n}^{2} \mid B\right] \stackrel{n \rightarrow \infty}{\longrightarrow} \iint_{\mathbb{R}^{2}} h(x, z) \pi(x \mid B) \nu_{2}(z) \mathrm{d} x \mathrm{~d} z .
$$

Thus $\mathbb{E}_{\pi(\cdot \mid B), q_{n}(\cdot \mid B)}\left[\rho_{n}^{2} \mid B\right]$ almost surely decays as $\sigma_{n}^{2 \beta}$.

The final task is to show that a Lindeberg-type condition holds almost surely.

LEMMA 22. Let the assumptions of Framework 15 be satisfied. Then almost surely (for almost every realisation of the random environment $B$ )

$$
\mathbb{E}\left[\mathbb{E}_{\pi(\cdot \mid B), q_{n}(\cdot \mid B)}\left[\rho_{n}^{2} 1_{\rho_{n}^{2}>\sigma_{n}^{\beta}} \mid B\right]^{m}\right] \precsim \sigma_{n}^{3 m \beta} .
$$

PROOF. By a combination of the Cauchy-Schwarz and Markov inequalities, for almost every realisation of $B$,

$$
\begin{aligned}
\mathbb{E}_{\pi(\cdot \mid B), q_{n}(\cdot \mid B)}\left[\rho_{n}^{2} 1_{\rho_{n}^{2}>\sigma_{n}^{\beta}} \mid B\right] & \leq \mathbb{E}_{\pi(\cdot \mid B), q_{n}(\cdot \mid B)}\left[\rho_{n}^{4} \mid B\right]^{1 / 2} \cdot \mathbb{P}_{\pi(\cdot \mid B), q_{n}(\cdot \mid B)}\left[\rho_{n}^{2}>\sigma_{n}^{\beta} \mid B\right]^{1 / 2} \\
& \leq \sigma_{n}^{-\beta} \mathbb{E}_{\pi(\cdot \mid B), q_{n}(\cdot \mid B)}\left[\rho_{n}^{4} \mid B\right] .
\end{aligned}
$$

Hence, by Jensen's inequality,

$$
\mathbb{E}\left[\mathbb{E}_{\pi(\cdot \mid B), q_{n}(\cdot \mid B)}\left[\rho_{n}^{2} 1_{\rho_{n}^{2}>\sigma_{n}^{\beta}} \mid B\right]^{m}\right] \leq \sigma_{n}^{-m a} \mathbb{E}\left[\mathbb{E}_{\pi(\cdot \mid B), q_{n}(\cdot \mid B)}\left[\rho_{n}^{4 m} \mid B\right]\right] .
$$
from

As in the case of $\mathcal{I}_{n}(B)$ (see (10)) the random functional $\mathbb{E}_{\pi(\cdot \mid B), q_{n}(\cdot \mid B)}\left[\rho_{n}^{4 m} \mid B\right]$ differs

$$
\iint_{\mathbb{R}^{2}} \rho_{n}^{4 m}\left(x, x+\sigma_{n} z \mid B\right) \xi(x \mid B) \sigma_{n} q_{n}\left(x, x+\sigma_{n} z \mid B\right) \mathrm{d} x \mathrm{~d} z
$$

just by a normalising constant. Using Assumptions 15.(A) and 15.(B), the Fubini-Tonelli theorem, and the Cauchy-Schwarz inequality twice over,

$$
\begin{aligned}
& \mathbb{E} {\left[\mathbb{E}_{\pi(\cdot \mid B), q_{n}(\cdot \mid B)}\left[\rho_{n}^{4 m} \mid B\right]\right] } \\
&= \mathbb{E}\left[\iint_{\mathbb{R}^{2}} \rho_{n}^{4 m}\left(x, x+\sigma_{n} z \mid B\right) \exp \left(K(x \mid B) L_{n}(x, z \mid B) v(x, z) \mathrm{d} x \mathrm{~d} z\right]\right. \\
& \leq \mathbb{E}\left[\iint_{\mathbb{R}^{2}} \rho_{n}^{8 m}\left(x, x+\sigma_{n} z \mid B\right) v(x, z) \mathrm{d} x \mathrm{~d} z\right]^{1 / 2} \times \mathbb{E}\left[\iint_{\mathbb{R}^{2}} \exp (4 K(x \mid B) v(x, z) \mathrm{d} x \mathrm{~d} z]^{1 / 4}\right. \\
& \quad \times \mathbb{E}\left[\iint_{\mathbb{R}^{2}} L_{n}^{4}(x, z \mid B) v(x, z) \mathrm{d} x \mathrm{~d} z\right]^{1 / 4} .
\end{aligned}
$$

The first factor is finite and decays as $\sigma_{n}^{4 \beta m}$ by Assumption 15.(C), the second is bounded because of Assumption 15.(A) and the third is bounded because of Assumption 15.(B). Hence, the result follows. 
COROLLARY 23. Let the assumptions of Framework 15 be satisfied. Then almost surely (for almost every realisation of the random environment $B$ )

$$
\sigma_{n}^{-2 \beta} \mathbb{E}_{\pi(\cdot \mid B), q_{n}(\cdot \mid B)}\left[\rho_{n}^{2} 1_{\rho_{n}^{2}>\sigma_{n}^{\beta}} \mid B\right] \stackrel{n \rightarrow \infty}{\longrightarrow} 0 .
$$

That is, $\mathbb{E}_{\pi(\cdot \mid B), q_{n}(\cdot \mid B)}\left[\rho_{n}^{2} 1_{\rho_{n}^{2}>\sigma_{n}^{\beta}} \mid B\right]$ almost surely decays faster than $\sigma_{n}^{2 \beta}$.

PROOF. Note that all integrands are strictly positive and use Lemma 22 together with Proposition 19 for $U_{n}=\mathbb{E}_{\pi(\cdot \mid B), q_{n}(\cdot \mid B)}\left[\rho_{n}^{2} 1_{\rho_{n}^{2}>\sigma_{n}^{\beta}} \mid B\right], \tilde{U}_{n}=0, \delta_{n}=\sigma_{n}^{2 \beta}$ and $\kappa:=\frac{1}{2}$. Note that $m \kappa=\frac{m}{2}>\frac{3}{2}>1$ by Assumption 15 .

THEOREM 24. Let the assumptions of Framework 15 be satisfied. For $n=1,2, \ldots$, and for each $\bar{x}=\left(x_{1}, \ldots, x_{n}\right) \in \mathbb{R}^{n}$, let $\Pi_{n}(\bar{x} \mid B)=\prod_{i=1}^{n} \pi\left(x_{i} \mid B\right)$ and $Q_{n}(\bar{x}, \mathrm{~d} \bar{y} \mid B)=$ $\prod_{i=1}^{n} q_{n}\left(x_{i}, y_{i} \mid B\right) \mathrm{d} y_{i}$ be respectively a target and a proposal on $\mathbb{R}^{n}$, both depending on a random environment $B$. If $X^{(n)}(B) \sim \Pi_{n}(\cdot \mid B)$ and $Y^{(n)}(B) \sim Q_{n}\left(X^{(n)}, \mathrm{d} \bar{y} \mid B\right)$ then there is $\sigma^{2}>0$ such that the Metropolis-Hastings acceptance probabilities (conditional on the underlying $B$ ) satisfy

$$
\alpha\left(X^{(n)}(B), Y^{(n)}(B)\right) \stackrel{w}{\rightarrow}(1 \wedge \exp )\left(N\left(-\frac{1}{2} \sigma^{2}, \sigma^{2}\right)\right) \text { as } n \rightarrow \infty,
$$

almost surely (almost surely in the random environment B). Moreover, we may take

$$
\sigma^{2}=\ell^{2 \beta} \iint_{\mathbb{R}^{2}} h(x, z) \pi(x \mid B) v_{2}(z) \mathrm{d} x \mathrm{~d} z
$$

PROOF. We restrict ourselves to the almost sure event of realisations of the random environment such that the conclusions of Corollary 21 and Corollary 23 both hold simultaneously. For notational convenience we fix an arbitrary realisation of the random environment $B$ satisfying this event and condition on this realization, and in the remainder of the proof we omit all reference to the random environment.

The $i$-th coordinates $X_{i}^{(n)}$ and $Y_{i}^{(n)}$ of $X^{(n)}$ and $Y^{(n)}$ are jointly distributed according to the product probability measure $\pi(x) q_{n}(x, y) \mathrm{d} x \mathrm{~d} y$. The product structure implies

$$
\Psi\left(X^{(n)}, Y^{(n)}\right):=\log \left(\frac{\Pi_{n}\left(Y^{(n)}\right) Q_{n}\left(Y^{(n)}, X^{(n)}\right)}{\Pi_{n}\left(X^{(n)}\right) Q_{n}\left(X^{(n)}, Y^{(n)}\right)}\right)=\sum_{i=1}^{n} \rho_{n}\left(X_{i}^{(n)}, Y_{i}^{(n)}\right) .
$$

Because of Corollary 21, if we set $\sigma_{n}=\ell n^{-\frac{1}{2 \beta}}$ then

$$
\begin{aligned}
& \mathbb{E}_{\pi q_{n}}\left[\sum_{i=1}^{n} \rho_{n}^{2}\left(X_{i}^{(n)}, Y_{i}^{(n)}\right)\right]=n \mathbb{E}_{\pi q_{n}}\left[\rho_{n}^{2}\right] \\
&=\ell^{2 \beta} \sigma_{n}^{-2 \beta} \mathbb{E}_{\pi q_{n}}\left[\rho_{n}^{2}\right] \\
& \stackrel{n \rightarrow \infty}{\longrightarrow} \sigma^{2}=\ell^{2 \beta} \iint_{\mathbb{R}^{2}} h(x, z) \pi(x) \nu_{2}(z) \mathrm{d} x \mathrm{~d} z .
\end{aligned}
$$

Moreover Corollary 21 and Corollary 23 imply that for each coordinate $\mathbb{E}_{\pi q_{n}}\left[\rho_{n}^{2}\left(X_{i}^{(n)}\right.\right.$, $\left.\left.Y_{i}^{(n)}\right)\right]=\mathbb{E}_{\pi q_{n}}\left[\rho_{n}^{2}\right]$ decays as $\sigma_{n}^{2 \beta}$, and $\mathbb{E}_{\pi q_{n}}\left[\rho_{n}^{2}\left(X_{i}^{(n)}, Y_{i}^{(n)}\right) 1_{\rho_{n}^{2}\left(X_{i}^{(n)}, Y^{(n)}\right)_{i}>\sigma_{n}^{\beta}}\right]=\mathbb{E}_{\pi q_{n}}\left[\rho_{n}^{2} \times\right.$ $\left.1_{\rho_{n}^{2}>\sigma_{n}^{\beta}}\right]$ decays faster than $\sigma_{n}^{2 \beta}$.

It is therefore a consequence of Theorem 8 that, as $n \rightarrow \infty$,

$$
\Psi\left(X^{(n)}, Y^{(n)}\right) \stackrel{w}{\rightarrow} N\left(-\frac{1}{2} \sigma^{2}, \sigma^{2}\right) .
$$


It is immediate from the definition of weak convergence that the desired result for acceptance probabilities follows, since $1 \wedge \exp$ is a bounded Lipschitz (hence continuous) function.

6. Application to random walk Metropolis algorithms. In this section we show that the anomalous scaling framework 15 of Section 5 holds for the Random walk Metropolis algorithm based on centered normal proposals when applied to a suitably perturbed product target. The perturbation is applied to the marginal log-density and corresponds to addition of a typical fBM path.

The random environment is given by a typical path of a two-sided fBM $B^{(H)}$ path with Hurst index $H \in(0,1)$. This is a stationary centred Gaussian process with covariance function given by equation (2) and with paths that are almost surely $\gamma$-Hölder continuous everywhere, for $0<\gamma<H$. In particular $B^{(H)}$ is a continuous Gaussian process with stationary increments.

As stipulated by Theorem 1, the RWM proposal is symmetric multivariate normal, with marginal probability density given by the kernel $q_{n}(x, d z)=\frac{1}{\sqrt{2 \pi \sigma_{n}^{2}}} \exp \left(-\frac{|z-x|^{2}}{2 \sigma_{n}^{2}}\right) \mathrm{d} z$, where $\sigma_{n}^{2}=\ell^{2} n^{-\frac{1}{2 H}}$ for some positive constant $\ell$. The reference measures $v_{1}(x)$ and $v_{2}(z)$ of the Framework 15 are both taken to be standard normal densities, so that $v(x, z)=v_{1}(x) v_{2}(z)=$ $\frac{1}{2 \pi} e^{-\frac{1}{2}\left(x^{2}+z^{2}\right)}$. We will also take $\beta=H$ and $\gamma=\min (H, 1-H)$. If it can be established that the assumptions listed in the Framework 15 all hold, then Theorem 1 will be an immediate consequence of Theorem 24.

The first task is to control the fluctuations of the potential given by the random environment $B^{(H)}$. As indicated above, we consider

$$
K\left(x \mid B^{(H)}\right)=B_{x}^{(H)} .
$$

LEMMA 25. Assumption 15.(A) is satisfied.

Proof. Evidently, $\left(K\left(x \mid B^{(H)}\right): x \in \mathbb{R}\right)$ is a centered Gaussian process, since it is simply fractional Brownian motion. Moreover its variance function is $k(x)=|x|^{2 H}$ (defined for every real $x)$. Assumption 15.(A) requires finiteness of $\mathbb{E}\left[\exp \left(m K\left(x \mid B^{(H)}\right)\right)\right]$ for some suitable $m$.

In fact for every real $m$, for any real $x$,

$$
\mathbb{E}\left[\exp \left(m K\left(x \mid B^{(H)}\right)\right)\right]=\frac{1}{\sqrt{2 \pi|x|^{2 H}}} \int_{\mathbb{R}} \exp \left(-\frac{y^{2}}{2|x|^{2 H}}+m y\right) \mathrm{d} y=e^{\frac{m^{2}|x|^{2 H}}{2}},
$$

and

$$
\int_{\mathbb{R}} e^{\frac{m^{2}|x|^{2 H}}{2}} v_{1}(x) \mathrm{d} x=\frac{1}{\sqrt{2 \pi}} \int_{\mathbb{R}} e^{\frac{m^{2}|x|^{2 H}}{2}-\frac{x^{2}}{2}} \mathrm{~d} x<\infty .
$$

For the RWM case the "asymptotic behaviour of proposal" property follows directly.

LEMMA 26. Assumption 15.(B) is satisfied.

PROOF. The RWM proposal is given by

$$
q_{n}(x, d z)=\frac{1}{\sqrt{2 \pi \sigma_{n}^{2}}} \exp \left(-\frac{(z-x)^{2}}{2 \sigma_{n}^{2}}\right) \mathrm{d} z
$$


so $\sigma_{n} q_{n}\left(x, x+\sigma_{n} z\right)=v_{2}(z)$ identically. Accordingly $L_{n}\left(x, z \mid B^{(H)}\right)=1$, and thus, for all $m$,

$$
\iint_{\mathbb{R}^{2}} \mathbb{E}\left[\left|L_{n}(x, z \mid B)-1\right|^{4 m}\right] v(x, z) \mathrm{d} x \mathrm{~d} z \equiv 0 .
$$

To establish the "approximate normality of LMHR" property we need to define

$$
M_{n}\left(x, z \mid B^{(H)}\right)=B_{x+\sigma_{n} z}^{(H)}-B_{x}^{(H)} .
$$

LEMMA 27. Assumption 15.(C) is satisfied if $M_{n}$ is defined using equation (22).

PRoOF. It is immediate that $\left(M_{n}\left(x, z \mid B^{(H)}\right): x \in \mathbb{R}\right)$ is a centred Gaussian process, since it is a linear transformation of fBM. Moreover it follows directly from the fBM covariance as given in Equation (2) that the variance of $M_{n}\left(x, z \mid B^{(H)}\right)$ is given by $|z|^{2 H} \sigma_{n}^{2 \beta}$ (bearing in mind that we have chosen $\beta=H$ ); and certainly $|z|^{2 H}$ is a function of polynomial growth.

Consider $\Delta_{n}\left(x, z \mid B^{(H)}\right)$ determined for all real $x, z$ and all positive integers $n$ by

$$
\rho_{n}\left(x, x+\sigma_{n} z \mid B^{(H)}\right)=M_{n}\left(x, z \mid B^{(H)}\right)+\Delta_{n}\left(x, z \mid B^{(H)}\right) .
$$

Since

$$
\rho_{n}\left(x, x+\sigma_{n} z \mid B^{(H)}\right)=\log \left(\frac{\xi\left(x+z \mid B^{(H)}\right)}{\xi\left(x \mid B^{(H)}\right)}\right)=B_{x+\sigma_{n} z}^{(H)}+\frac{\left(x+\sigma_{n} z\right)^{2}}{2}-B_{x}^{(H)}-\frac{x^{2}}{2},
$$

we obtain

$$
\Delta_{n}\left(x, z \mid B^{(H)}\right)=\frac{1}{2}\left(x^{2}-\left(x+\sigma_{n} z\right)^{2}\right)=-\sigma_{n} z\left(x+\frac{1}{2} \sigma_{n} z\right) .
$$

Accordingly it follows that, for some constant $C_{m}$ depending only on $m$,

$$
\begin{aligned}
\iint_{\mathbb{R}^{2}} \mathbb{E}\left[\left|\Delta_{n}(x, z \mid B)\right|^{8 m}\right] \nu(x, z) \mathrm{d} x \mathrm{~d} z & =\frac{1}{2 \pi} \iint_{\mathbb{R}^{2}}\left|\sigma_{n} z\left(x+\frac{1}{2} \sigma_{n} z\right)\right|^{8 m} e^{-\frac{1}{2}\left(x^{2}+z^{2}\right)} \mathrm{d} x \mathrm{~d} z \\
& \leq C_{m} \times \sigma_{n}^{8 m} \precsim \sigma_{n}^{8 m \beta+8 m \gamma},
\end{aligned}
$$

since $\beta+\gamma=H+\min (H, 1-H) \leq 1$ for $H \in(0,1)$.

Finally, to demonstrate the "asymptotic weak dependence" property we define the following subsets of $\mathbb{R}^{4}$ :

$$
\mathcal{S}_{n}:=\left\{\left(x_{1}, z_{1}, x_{2}, z_{2}\right) \in \mathbb{R}^{4}:\left|x_{1}-x_{2}\right|>2 \sigma_{n}^{\frac{1}{2}}\left(\left|z_{1}\right|+\left|z_{2}\right|\right)\right\}
$$

LEMMA 28. Assumption 15.(D) is satisfied using the sets $\mathcal{S}_{n}$ :

(i) $\int_{\mathcal{S}_{n}^{c}} v\left(x_{1}, z_{1}\right) v\left(x_{2}, z_{2}\right) \mathrm{d} x_{1} \mathrm{~d} z_{1} \mathrm{~d} x_{2} \mathrm{~d} z_{2} \precsim \sigma_{n}^{\frac{1}{2}}$.

(ii) For any $\left(x_{1}, x_{2}, z_{1}, z_{2}\right) \in \mathcal{S}_{n}$, noting that $2 \beta+\gamma=2 H+\min (H, 1-H) \leq 1+H$ if $H \in(0,1)$,

$$
\left|\mathbb{E}\left[M_{n}\left(x_{1}, z_{1}\right) M_{n}\left(x_{2}, z_{2}\right)\right]\right| \leq \frac{H|2 H-1|}{2^{2-2 H}}\left|z_{1}\right|^{H}\left|z_{2}\right|^{H} \sigma_{n}^{1+H} \leq \frac{H|2 H-1|}{2^{2-2 H}}\left|z_{1}\right|^{H}\left|z_{2}\right|^{H} \sigma_{n}^{2 \beta+\gamma} .
$$

(iii) Noting again that $\beta+\gamma=H+\min (H, 1-H)$ for $H \in(0,1)$, there exists a polynomial $g_{2}\left(x_{1}, z_{1}, x_{2}\right)$ such that

$$
\left|\mathbb{E}\left[M_{n}\left(x_{1}, z_{1} \mid B^{(H)}\right) K\left(x_{2} \mid B^{(H)}\right)\right]\right| \leq g_{2}\left(x_{1}, z_{1}, x_{2}\right) \sigma_{n}^{H} \cdot \sigma_{n}^{\min (H, 1-H)}=g_{2}\left(x_{1}, z_{1}, x_{2}\right) \sigma_{n}^{\beta+\gamma} .
$$


PROOF. Property (i) follows by applying Lemma A.3 in the Appendix, using the sequence $a_{n}=\sigma_{n}^{\frac{1}{2}}$.

For (ii) first note that by (2) the expectation $\mathbb{E}\left[M_{n}\left(x_{1}, z_{1}\right) M_{n}\left(x_{2}, z_{2}\right)\right]$ can be rewritten as $-\frac{1}{2}\left(\left|x_{1}-x_{2}+\sigma_{n} z_{1}-\sigma_{n} z_{2}\right|^{2 H}-\left|x_{1}-x_{2}-\sigma_{n} z_{2}\right|^{2 H}-\left|x_{1}-x_{2}+\sigma_{n} z_{1}\right|^{2 H}+\left|x_{1}-x_{2}\right|^{2 H}\right)$.

Consider $\left(x_{1}, x_{2}, z_{1}, z_{2}\right) \in \mathcal{S}_{n}$, and apply Lemma A.3 with $u=v=\sigma_{n}^{\frac{1}{2}}$ (assuming $n$ large enough that $\left.\sigma_{n} \leq 1\right)$. It follows that

$$
\left.\max \left(\left|\sigma_{n} z_{1}-\sigma_{n} z_{2}\right|,\left|\sigma_{n} z_{1}\right|, \mid \sigma_{n} z_{2}\right) \mid\right)<\frac{\left|x_{1}-x_{2}\right|}{2}
$$

and

$$
\min \left(\left|x_{1}-x_{2}+\sigma_{n} z_{1}-\sigma_{n} z_{2}\right|,\left|x_{1}-x_{2}+\sigma_{n} z_{1}\right|,\left|x_{1}-x_{2}-\sigma_{n} z_{2}\right|,\left|x_{1}-x_{2}\right|\right)>\frac{\left|x_{1}-x_{2}\right|}{2} .
$$

Hence, $x_{1}-x_{2}+\sigma_{n} z_{1}-\sigma_{n} z_{2}, x_{1}-x_{2}+\sigma_{n} z_{1}, x_{1}-x_{2}-\sigma_{n} z_{2}$ and $x_{1}-x_{2}$ are either all positive or all negative. Consequently the function $x \mapsto|x|^{2 H}$ is smooth over any of the bounded intervals with endpoints drawn from these four points, and so we may apply Lemma A.1 to argue

$$
\begin{aligned}
\mid \mathbb{E} & {\left[M_{n}\left(x_{1}, z_{1} \mid B^{(H)}\right) M_{n}\left(x_{2}, z_{2} \mid B^{(H)}\right)\right] \mid } \\
& \leq H \times|2 H-1| \times\left|z_{1}\right|\left|z_{2}\right| \sigma_{n}^{2} \int_{0}^{1} \int_{0}^{1}\left|x_{1}-x_{2}+u \sigma_{n} z_{1}-v \sigma_{n} z_{2}\right|^{2 H-2} \mathrm{~d} u \mathrm{~d} v \\
& \leq H \times|2 H-1| \times\left|z_{1} z_{2}\right|\left|\frac{x_{1}-x_{2}}{2}\right|^{2 H-2} \sigma_{n}^{2} \\
& =H \times|2 H-1| \times\left|z_{1}\right|^{H}\left|z_{2}\right|^{H}\left(\frac{2 \sqrt{\left|z_{1}\right|\left|z_{2}\right|}}{\left|x_{1}-x_{2}\right|}\right)^{2-2 H} \sigma_{n}^{2} \\
& \leq H \times|2 H-1| \times\left|z_{1}\right|^{H}\left|z_{2}\right|^{H}\left(\frac{\left|z_{1}\right|+\left|z_{2}\right|}{\left|x_{1}-x_{2}\right|}\right)^{2-2 H} \sigma_{n}^{2} \leq \frac{H \times|2 H-1|}{2^{2-2 H}}\left|z_{1}\right|^{H}\left|z_{2}\right|^{H} \sigma_{n}^{1+H} .
\end{aligned}
$$

Here the last step follows because of the definition of $\mathcal{S}_{n}$ : if $\left(x_{1}, x_{2}, z_{1}, z_{2}\right) \in \mathcal{S}_{n}$ then $2 \sigma_{n}^{\frac{1}{2}}\left(\left|z_{1}\right|+\left|z_{2}\right|\right)<\left|x_{1}-x_{2}\right|$.

For (iii), first observe that by (2)

$$
\begin{aligned}
\mathbb{E} & {\left[M_{n}\left(x_{1}, z_{1} \mid B^{(H)}\right) K\left(x_{2} \mid B^{(H)}\right)\right] } \\
& =\mathbb{E}\left[\left(B_{x_{1}+\sigma_{n} z_{1}}^{(H)}-B_{x_{1}}^{(H)}\right) B_{x_{2}}^{(H)}\right] \\
& =\frac{1}{2}\left(\left|x_{1}+\sigma_{n} z_{1}\right|^{2 H}-\left|x_{1}\right|^{2 H}-\left|x_{1}-x_{2}+\sigma_{n} z_{1}\right|^{2 H}+\left|x_{1}-x_{2}\right|^{2 H}\right) .
\end{aligned}
$$

We now need to distinguish between the cases $H \lesseqgtr \frac{1}{2}$. First, consider the case $H \leq 1 / 2$, so that $2 H \leq 1, H+\min (H, 1-H)=2 H$, and $\left.|| a\right|^{2 H}-|b|^{2 H}|\leq| a-\left.b\right|^{2 H}$ holds for real $a, b$. Consequently,

$$
\left|\mathbb{E}\left[M_{n}\left(x_{1}, z_{1} \mid B^{(H)}\right) K\left(x_{2} \mid B^{(H)}\right)\right]\right| \leq \sigma_{n}^{2 H}\left|z_{1}\right|^{2 H}=\left|z_{1}\right|^{2 H} \sigma_{n}^{H+\min (H, 1-H)}=\left|z_{1}\right|^{2 H} \sigma_{n}^{\beta+\gamma} .
$$

Second, consider the case $H>1 / 2$, so that $1<2 H<2$ and the function $x \mapsto|x|^{2 H}$ is in $\mathcal{C}^{1}(\mathbb{R})$. Then for any real $a, b$, using $|a|^{2 H-1}<1+|a|$,

$$
\begin{aligned}
|| a+\left.b\right|^{2 H}-|a|^{2 H} \mid & =2 H\left|b \int_{0}^{1}\right| a+\left.u b\right|^{2 H-1} \operatorname{sign}(a+u b) \mathrm{d} u \mid \\
& \leq 2 H|b| \int_{0}^{1}|a+u b|^{2 H-1} \mathrm{~d} u \leq 2 H|b|(2+|a|+|b|),
\end{aligned}
$$


and hence

$$
\left|\mathbb{E}\left[M_{n}\left(x_{1}, z_{1} \mid B^{(H)}\right) K\left(x_{2} \mid B^{(H)}\right)\right]\right| \leq H \sigma_{n}\left|z_{1}\right|\left(4+\left|x_{1}\right|+\left|x_{1}-x_{2}\right|+2 \sigma_{n}\left|z_{1}\right|\right) .
$$

The proof of Theorem 1 is now immediate.

Proof of TheOREM 1. Lemmas 25, 26, 27 and 28 together show that the anomalous scaling framework 15 holds for the RWM algorithm as described at the head of this section and as stipulated by Theorem 1 . Consequently Theorem 1 is a direct consequence of Theorem 24.

7. Application to Metropolis adjusted Langevin algorithms. In this section we show that the anomalous scaling framework 15 of Section 5 holds for the Metropolis adjusted Langevin algorithm based on normal proposals when applied to a suitably perturbed product target. The perturbation is applied at the level of the second derivative of the log-density of the marginal target, adding a typical fBM path multiplied by a nonrandom localization term.

Again the random environment is given by a typical path of a two-sided fBM $B^{(H)}$ process with Hurst index $H \in(0,1)$, a continuous Gaussian process with stationary increments.

As stipulated by Theorem 2, the MALA proposal has probability density $q_{n}\left(x, d y \mid B^{(H)}, c\right)$ given by the multivariate normal density

$$
N\left(x+\frac{\sigma_{n}^{2}}{2} \nabla\left(\log \pi\left(x \mid B^{(H)} ; c\right)\right), \sigma_{n}^{2} \cdot I_{n}\right),
$$

where $\sigma_{n}^{2}=\ell^{2} n^{-\frac{1}{4+2 H}}$ for some positive constant $\ell$. Here $c$ refers to the constant used for the definition of localization in equation (3). Again the reference measures $v_{1}(x)$ and $v_{2}(z)$ of the Framework 15 are both taken to be standard normal densities, so that $v(x, z)=v_{1}(x) v_{2}(z)=$ $\frac{1}{2 \pi} e^{-\frac{1}{2}\left(x^{2}+z^{2}\right)}$. For MALA we take $\beta=2+H$ and $\gamma=\min (H, 1-H)$. Once again we need to establish that the anomalous scaling framework 15 holds; Theorem 2 will then follow using Theorem 24.

We begin by showing that the log-target density has normal fluctuations. To that end define

$$
K\left(x \mid B^{(H)} ; c\right)=x^{2} \int_{0}^{1} B_{x s}^{(H)} \varphi_{c}(x s)(1-s) \mathrm{d} s=\int_{0}^{x} B_{u}^{(H)} \varphi_{c}(u)(x-u) \mathrm{d} u,
$$

where $\varphi_{c}(x)=\min \left\{1, c^{\frac{3}{2 H}}|x|^{-3}\right\}$ is the localisation function introduced in Section 2 by equation (3). (The last expression above is obtained by using the substitution $u=s x$.) It is convenient to focus on potentials (the log-marginal target probability densities), which are given by

$$
\begin{aligned}
V\left(x \mid B^{(H)} ; c\right) & =\log \left(\pi\left(x \mid B^{(H)} ; c\right)\right) \\
& =-\log \left(\int_{\mathbb{R}} \xi\left(u \mid B^{(H)} ; c\right) \mathrm{d} u\right)-\frac{x^{2}}{2}+\int_{0}^{x} B_{u}^{(H)} \varphi_{c}(u)(x-u) \mathrm{d} u .
\end{aligned}
$$

Repeated differentiation yields formulae for first and second derivatives of the potential:

$$
\begin{aligned}
& \dot{V}\left(x \mid B^{(H)} ; c\right)=-x+\int_{0}^{x} B_{u}^{(H)} \varphi_{c}(u) \mathrm{d} u, \\
& \ddot{V}\left(x \mid B^{(H)} ; c\right)=-1+B_{x}^{(H)} \varphi_{c}(x) .
\end{aligned}
$$

We first establish some basic properties for the localisation function $\varphi_{c}(x)$.

LEMMA 29. The localisation function $\varphi_{c}(x)=\min \left\{1, c^{\frac{3}{2 H}}|x|^{-3}\right\}$ satisfies the following: 
(i) $\varphi_{c}(x) \leq 1$ for all $x \in \mathbb{R}$.

(ii) $|x|^{2 H} \varphi_{c}(x) \leq c$ for all $x \in \mathbb{R}$.

(iii) $\varphi_{c}$ is Lipschitz with constant $3 c^{-\frac{1}{2 H}}$.

PROOF. Property (i) follows immediately from the definition. Property (ii) follows by arguing separately for $|x| \leq c^{\frac{1}{2 H}}$, when $|x|^{2 H} \varphi_{c}(x)=|x|^{2 H} \leq c$, and $|x|>c^{\frac{1}{2 H}}$, when $|x|^{2 H} \varphi_{c}(x)<|x|^{2 H-3} c^{\frac{3}{2 H}}<c^{1-3 /(2 H)} c^{\frac{3}{2 H}}=c$ (note that $H \in(0,1)$ ). Property (iii) follows from considering the derivative of the continuous function $\varphi_{c}(x)$ away from the gradient discontinuities at $x= \pm c^{\frac{1}{2 H}}$.

We first consider the "mixed Gaussian perturbation" property.

LEMMA 30. For any positive integer m, Assumption 15.(A) is satisfied for all sufficiently small localisation parameters $c>0$ :

(i) For every real $x$, set

$$
\xi\left(x \mid B^{(H)} ; c\right)=\exp \left(K\left(x \mid B^{(H)} ; c\right)\right) v_{1}(x)
$$

Then $K\left(x \mid B^{(H)} ; c\right)$ is a centred normal random variable with variance $k(x) \leq 3(1+$ $\left.\frac{1}{2-2 H}\right) c^{1+\frac{1}{H} \cdot x^{2}}$.

(ii) For any real $x$ it is the case that $\exp \left(2 m^{2} k(x)\right) \leq e^{6 m^{2}\left(1+\frac{1}{2-2 H}\right) c^{1+\frac{1}{H}} \cdot x^{2}}$ and this is integrable with respect to $v_{1}$ for all sufficiently small $c>0$.

PROOF. Normality in point (i) follows immediately from (25) and the observation that $B^{(H)}$ is a zero-mean Gaussian process. The rest of property (i) is trivially true if $x=0$, since

$$
V\left(0 \mid B^{(H)} ; c\right)=-\log \left(\int_{\mathbb{R}} \xi\left(u \mid B^{(H)} ; c\right) \mathrm{d} u\right)
$$

is just the $\log$ of the normalising constant, so we need only deal with $x \neq 0$. Note that the inequality $\left|\Gamma^{(H)}(x, y)\right| \leq|x|^{2 H}+|y|^{2 H}$ (see (2)) implies

$$
\begin{aligned}
\mathbb{E}\left[\left(K\left(x \mid B^{(H)} ; c\right)\right)^{2}\right] & =x^{4} \int_{0}^{1} \int_{0}^{1} \Gamma^{(H)}(s x, t x) \varphi_{c}(t x) \varphi_{c}(s x)(1-s)(1-t) \mathrm{d} s \mathrm{~d} t \\
& \leq 2 x^{4} \cdot \int_{0}^{1} \varphi_{c}(t x)(1-t) \mathrm{d} t \cdot \int_{0}^{1}|s x|^{2 H} \varphi_{c}(s x)(1-s) \mathrm{d} s .
\end{aligned}
$$

The definition (3) of the localisation function permits the bound

$$
\begin{aligned}
\int_{0}^{1} \varphi_{c}(t x)(1-t) \mathrm{d} t & =\int_{0}^{c^{\frac{1}{2 H}}|x|^{-1}} \varphi_{c}(t x)(1-t) \mathrm{d} t+\int_{c^{\frac{1}{2 H}}|x|^{-1}}^{1} \varphi_{c}(t x)(1-t) \mathrm{d} t \\
& \leq \int_{0}^{c^{\frac{1}{2 H}}|x|^{-1}} \mathrm{~d} t+c^{\frac{3}{2 H}}|x|^{-3} \int_{c^{\frac{1}{2 H}}|x|^{-1}}^{\infty} t^{-3} \mathrm{~d} t \leq \frac{3}{2} c^{\frac{1}{2 H}}|x|^{-1}
\end{aligned}
$$


Splitting the integral $\int_{0}^{1}|s x|^{2 H} \varphi_{c}(x s)(1-s) \mathrm{d} s$ and employing Lemma 29(ii) and (3) respectively to the two parts (and noting again that $H \in(0,1)$ ), we obtain

$$
\begin{aligned}
\int_{0}^{1}|s x|^{2 H} \varphi_{c}(x s)(1-s) \mathrm{d} s= & \int_{0}^{c^{\frac{1}{2 H}}|x|^{-1}}|s x|^{2 H} \varphi_{c}(s x)(1-s) \mathrm{d} s \\
& +\int_{c^{\frac{1}{2 H}}|x|^{-1}}^{1}|s x|^{2 H} \varphi_{c}(s x)(1-s) \mathrm{d} s \\
\leq & \left(1+\frac{1}{2-2 H}\right) c^{1+\frac{1}{2 H}|x|^{-1}} .
\end{aligned}
$$

The remainder of property (i) is now established by substituting (30) and (31) into (29).

Finally, property (ii) is established by applying property (i) to bound

$$
\exp \left(2 m^{2} k(x)\right) \leq \exp \left(6 m^{2} \cdot\left(1+\frac{1}{2-2 H}\right) c^{1+\frac{1}{H}} x^{2}\right)
$$

Thus property (ii) holds when $6 m^{2}\left(1+\frac{1}{2-2 H}\right) c^{1+\frac{1}{H}}<\frac{1}{2}$, which is to say when $c<\left(12 m^{2}(1+\right.$ $\left.\left.\frac{1}{2-2 H}\right)\right)^{-\frac{H}{1+H}}$.

We now establish the "asymptotic behaviour of proposal" property. We begin by considering the variance and exponential moments of the first derivative of the potential.

LEMMA 31. The following statements hold:

(i) $\dot{V}\left(x \mid B^{(H)} ; c\right)+x$ is a centred normal random variable with variance controlled for every real $x$ by

$$
\mathbb{E}\left[\left(\dot{V}\left(x \mid B^{(H)} ; c\right)+x\right)^{2}\right] \leq 3\left(1+\frac{1}{2-2 H}\right) c^{1+\frac{1}{H}} .
$$

(ii) For every real $x, z$,

$$
\begin{aligned}
\mathbb{E} & {\left[\exp \left(8 m \sigma_{n}\left|z \dot{V}\left(x \mid B^{(H)} ; c\right)\right|\right)\right] } \\
& \leq 2 \exp \left(4 m \sigma_{n} x^{2}\right) \exp \left(4 m \sigma_{n} z^{2}\right) \exp \left(32 m^{2} \sigma_{n}^{2} z^{2} \mathbb{E}\left[\left(\dot{V}\left(x \mid B^{(H)} ; c\right)+x\right)^{2}\right]\right) .
\end{aligned}
$$

Furthermore there is a convenient bound for all sufficiently large $n$ :

$$
\mathbb{E}\left[\exp \left(8 m \sigma_{n}\left|z \dot{V}\left(x \mid B^{(H)} ; c\right)\right|\right)\right] \leq 2 \exp \left(\frac{2 x^{2}}{3}\right) \exp \left(\frac{4 z^{2}}{3}\right) .
$$

(iii) For all sufficiently large $n, \mathbb{E}\left[\exp \left(m \sigma_{n}^{2} \dot{V}\left(x \mid B^{(H)} ; c\right)^{2}\right)\right] \leq \sqrt{2} \exp \left(\frac{2 x^{2}}{3}\right)$ for all real $x$.

PROOF. Normality in property (i) follows immediately from (27) and the observation that $B^{(H)}$ is a zero-mean Gaussian process. The proof of the bound is entirely analogous to the proof of Lemma 30(i). Proof of property (ii): this uses property (i), the bounds $e^{|a u|} \leq$ $e^{|a| u}+e^{-|a| u}$ and $|z x| \leq \frac{1}{2}\left(x^{2}+z^{2}\right)$, and the fact that $\dot{V}\left(x \mid B^{(H)} ; c\right)+x$ is a centred normal random variable and therefore has zero mean:

$$
\begin{aligned}
\mathbb{E} & {\left[\exp \left(8 m \sigma_{n}\left|z \dot{V}\left(x \mid B^{(H)} ; c\right)\right|\right)\right] } \\
& \leq \exp \left(8 m \sigma_{n}|z x|\right) \mathbb{E}\left[\exp \left(8 m \sigma_{n}|z|\left|\dot{V}\left(x \mid B^{(H)} ; c\right)+x\right|\right)\right] \\
& \leq 2 \exp \left(8 m \sigma_{n}|z x|\right) \mathbb{E}\left[\exp \left(8 m \sigma_{n}|z|\left(\dot{V}\left(x \mid B^{(H)} ; c\right)+x\right)\right)\right] \\
& \leq 2 \exp \left(4 m \sigma_{n} x^{2}\right) \exp \left(4 m \sigma_{n} z^{2}\right) \exp \left(32 m^{2} \sigma_{n}^{2} z^{2} \mathbb{E}\left[\left(\dot{V}\left(x \mid B^{(H)} ; c\right)+x\right)^{2}\right]\right) .
\end{aligned}
$$


Here the second step uses $e^{|a u|} \leq e^{|a| u}+e^{-|a| u}$ and the symmetry of the random variable $\dot{V}\left(x \mid B^{(H)} ; c\right)+x$, while the last step also employs the formula for the moment generating function of a centred Gaussian random variable.

The rest of property (ii) follows by using property (i) for sufficiently large $n$.

Proof of property (iii): Take $n$ large enough (noting that $\left.\sigma_{n} \rightarrow 0\right)$ so that $24 m \sigma_{n}^{2}(1+$ $\left.\frac{1}{2-2 H}\right) c^{1+\frac{1}{H}} \leq \frac{1}{2}$ and also $4 m \sigma_{n}^{2}<\frac{2}{3}$. Using $a^{2} \leq 2 b^{2}+2(a-b)^{2}$, and bearing in mind the bound of property (i),

$$
\begin{aligned}
\mathbb{E} & {\left[\exp \left(2 m \sigma_{n}^{2} \dot{V}\left(x \mid B^{(H)} ; c\right)^{2}\right)\right] } \\
& \leq \exp \left(4 m \sigma_{n}^{2} x^{2}\right) \mathbb{E}\left[\exp \left(4 m \sigma_{n}^{2}\left(\dot{V}\left(x \mid B^{(H)} ; c\right)+x\right)^{2}\right)\right] \\
& =\exp \left(4 m \sigma_{n}^{2} x^{2}\right)\left(1-8 m \sigma_{n}^{2} \mathbb{E}\left[\left(\dot{V}\left(x \mid B^{(H)} ; c\right)+x\right)^{2}\right]\right)^{-1 / 2} \\
& \leq \exp \left(4 m \sigma_{n}^{2} x^{2}\right)\left(1-24 m \sigma_{n}^{2}\left(1+\frac{1}{2-2 H}\right) c^{1+\frac{1}{H}}\right)^{-1 / 2} \leq \sqrt{2} \exp \left(\frac{2 x^{2}}{3}\right),
\end{aligned}
$$

where the last line uses the evaluation $\mathbb{E}\left[e^{\lambda^{2} N^{2}}\right]=\left(1-2 \lambda^{2}\right)^{-\frac{1}{2}}$ for $2 \lambda^{2}<1$ when $N$ is a standard normal random variable.

LEMMA 32. Assumption 15.(B) is satisfied.

(i) For every real $x, z$ and every positive integer $n$,

$$
\sigma_{n} q_{n}\left(x, x+\sigma_{n} z\right)=L_{n}\left(x, z \mid B^{(H)} ; c\right) v_{2}(z),
$$

where $L_{n}\left(x, z \mid B^{(H)} ; c\right)=\exp \left(\frac{z \sigma_{n}}{2} \dot{V}\left(x \mid B^{(H)} ; c\right)-\frac{\sigma_{n}^{2}}{8} \dot{V}\left(x \mid B^{(H)} ; c\right)^{2}\right)$.

(ii) Recall that we have stipulated $\gamma=\min (H, 1-H)$. The random variable $L_{n}(x, z \mid$ $\left.B^{(H)} ; c\right)$ satisfies

$$
\iint_{\mathbb{R}^{2}} \mathbb{E}\left[\left|L_{n}\left(x, z \mid B^{(H)} ; c\right)-1\right|^{4 m}\right] v(x, z) \mathrm{d} x \mathrm{~d} z \precsim \sigma_{n}^{4 m} \precsim \sigma_{n}^{4 m \gamma} .
$$

PROOF. Property (i) holds by definition, since

$$
\begin{aligned}
\sigma_{n} q_{n}\left(x, x+\sigma_{n} z\right) & =\frac{\sigma_{n}}{\sigma_{n} \sqrt{2 \pi}} \exp \left(-\frac{1}{2 \sigma_{n}^{2}}\left(\sigma_{n} z-\frac{\sigma_{n}^{2}}{2} \dot{V}\left(x \mid B^{(H)} ; c\right)\right)^{2}\right) \\
& =\frac{1}{\sqrt{2 \pi}} \exp \left(-\frac{1}{2}\left(z-\frac{\sigma_{n}}{2} \dot{V}\left(x \mid B^{(H)} ; c\right)\right)^{2}\right) \\
& =\frac{1}{\sqrt{2 \pi}} e^{-\frac{z^{2}}{2}} \cdot \exp \left(\frac{z \sigma_{n}}{2} \dot{V}\left(x \mid B^{(H)} ; c\right)-\frac{\sigma_{n}^{2}}{8} \dot{V}\left(x \mid B^{(H)} ; c\right)^{2}\right) .
\end{aligned}
$$

To see (ii) note that $\left|e^{t}-1\right| \leq|t| e^{|t|}$ holds for all $t \in \mathbb{R}$. Using this together with a repeated application of the Cauchy-Schwarz inequality, note that for all large enough $n$

$$
\begin{aligned}
\mathbb{E}\left[\left|L_{n}\left(x, z \mid B^{(H)} ; c\right)-1\right|^{4 m}\right]= & \mathbb{E}\left[\left|\exp \left(z \frac{\sigma_{n}}{2} \dot{V}\left(x \mid B^{(H)} ; c\right)-\frac{\sigma_{n}^{2}}{8} \dot{V}\left(x \mid B^{(H)} ; c\right)^{2}\right)-1\right|^{4 m}\right] \\
\leq & \mathbb{E}\left[\left(z \frac{\sigma_{n}}{2} \dot{V}\left(x \mid B^{(H)} ; c\right)-\frac{\sigma_{n}^{2}}{8} \dot{V}\left(x \mid B^{(H)} ; c\right)^{2}\right)^{4 m}\right. \\
& \left.\times \exp \left(2 m \sigma_{n}\left|z \dot{V}\left(x \mid B^{(H)} ; c\right)\right|\right) \exp \left(\frac{m \sigma_{n}^{2}}{2} \dot{V}\left(x \mid B^{(H)} ; c\right)^{2}\right)\right]
\end{aligned}
$$




$$
\begin{aligned}
\leq & \sigma_{n}^{4 m} \times \mathbb{E}\left[\left(\frac{z}{2} \dot{V}\left(x \mid B^{(H)} ; c\right)-\frac{\sigma_{n}}{8} \dot{V}\left(x \mid B^{(H)} ; c\right)^{2}\right)^{8 m}\right]^{1 / 2} \\
& \times \mathbb{E}\left[\exp \left(8 m \sigma_{n}\left|z \dot{V}\left(x \mid B^{(H)} ; c\right)\right|\right)\right]^{1 / 4} \\
& \times \mathbb{E}\left[\exp \left(2 m \sigma_{n}^{2} \dot{V}\left(x \mid B^{(H)} ; c\right)^{2}\right)\right]^{1 / 4}
\end{aligned}
$$

By Lemma $31, \dot{V}\left(x \mid B^{(H)} ; c\right)$ is a normal with mean $x$ and bounded variance, hence the first expectation can be controlled by a polynomial $g(x, z)$. The second expectation is bounded by $2^{1 / 4} e^{\frac{x^{2}}{6}} e^{\frac{z^{2}}{3}}$ by Lemma 31(ii), and the third is bounded by $2^{1 / 8} e^{\frac{x^{2}}{6}}$ by Lemma 31(iii). The proof is completed by observing that $g(x, z) e^{\frac{x^{2}}{3}} e^{\frac{z^{2}}{3}}$ is integrable with respect to the reference density $v$, since $v(x, z)=\frac{1}{2 \pi} e^{-\frac{1}{2}\left(x^{2}+z^{2}\right)}$.

To establish the "approximate LMHR normality" property, set

$$
M_{n}\left(x, z \mid B^{(H)} ; c\right)=\sigma_{n}^{2} z^{2} \int_{0}^{1} B_{x+t \sigma_{n} z}^{(H)} \varphi_{c}(x)(1-2 t) \mathrm{d} t .
$$

LEMMA 33. Assumption 15.(C) is satisfied:

(i) For all real $x, z$ and positive integers $n$

$$
\rho_{n}\left(x, x+\sigma_{n} z \mid B^{(H)} ; c\right)=M_{n}\left(x, z \mid B^{(H)} ; c\right)+\Delta_{n}\left(x, z \mid B^{(H)} ; c\right)
$$

for $\Delta_{n}\left(x, z \mid B^{(H)} ; c\right)=\Delta_{n}^{(1)}\left(x, z \mid B^{(H)} ; c\right)-\Delta_{n}^{(2)}\left(x, z \mid B^{(H)} ; c\right)$, where

$$
\begin{aligned}
& \Delta_{n}^{(1)}\left(x, z \mid B^{(H)} ; c\right)=\frac{\sigma_{n}^{2} z^{2}}{2} \int_{0}^{1} B_{x+t \sigma_{n} z}^{(H)}\left(\varphi_{c}\left(x+t \sigma_{n} z\right)-\varphi_{c}(x)\right)(1-2 t) \mathrm{d} t, \\
& \Delta_{n}^{(2)}\left(x, z \mid B^{(H)} ; c\right)=\frac{\sigma_{n}^{3} z}{4} \int_{0}^{1} \dot{V}\left(x+t \sigma_{n} z \mid B^{(H)} ; c\right) \ddot{V}\left(x+t \sigma_{n} z \mid B^{(H)} ; c\right) \mathrm{d} t .
\end{aligned}
$$

(ii) $\left(M_{n}\left(x, z \mid B^{(H)} ; c\right): x, z \in \mathbb{R}\right)$ is a centred Gaussian process with one-point variance $h(x, z) \sigma_{n}^{4+2 H}$ where

$$
h(x, z)=\frac{1}{2} \frac{H}{2+7 H+7 H^{2}+2 H^{3}} \varphi_{c}^{2}(x)|z|^{4+2 H} .
$$

(iii) Finally, recall $\beta=2+H$ and $\gamma=\min (H, 1-H)$ implying $8 \beta+8 \gamma \leq 24$ and

$$
\iint_{\mathbb{R}^{2}}\left|\Delta_{n}^{(1)}\left(x, z \mid B^{(H)} ; c\right)\right|^{8 m} v(x, z) \mathrm{d} x \mathrm{~d} z \precsim \sigma_{n}^{24 m} \precsim \sigma^{8 m \beta+8 m \gamma}
$$

and

$$
\iint_{\mathbb{R}^{2}}\left|\Delta_{n}^{(2)}\left(x, z \mid B^{(H)} ; c\right)\right|^{8 m} v(x, z) \mathrm{d} x \mathrm{~d} z \precsim \sigma_{n}^{24 m} \precsim \sigma^{8 m \beta+8 m \gamma} .
$$

Proof. We know that by formulae (26), and (24) and $a^{2}-b^{2}=(a+b)(a-b)$,

$$
\begin{aligned}
\rho_{n} & \left(x, x+\sigma_{n} z \mid B^{(H)} ; c\right) \\
= & \log \left(\pi\left(x+\sigma_{n} z \mid B^{(H)} ; c\right)\right)-\log \left(\pi\left(x \mid B^{(H)} ; c\right)\right) \\
& \quad+\log \left(q_{n}\left(x+\sigma_{n} z, x \mid B^{(H)} ; c\right)\right)-\log \left(q_{n}\left(x, x+\sigma_{n} z \mid B^{(H)} ; c\right)\right) \\
= & V\left(x+\sigma_{n} z \mid B^{(H)} ; c\right)-V\left(x \mid B^{(H)} ; c\right)
\end{aligned}
$$




$$
\begin{aligned}
& -\frac{1}{2 \sigma_{n}^{2}}\left(-\sigma_{n} z-\frac{\sigma_{n}^{2}}{2} \dot{V}\left(x+\sigma_{n} z \mid B^{(H)} ; c\right)\right)^{2}+\frac{1}{2 \sigma_{n}^{2}}\left(\sigma_{n} z-\frac{\sigma_{n}^{2}}{2} \dot{V}\left(x \mid B^{(H)} ; c\right)\right)^{2} \\
= & V\left(x+\sigma_{n} z \mid B^{(H)} ; c\right)-V\left(x \mid B^{(H)} ; c\right) \\
& -\frac{\sigma_{n} z}{2}\left(\dot{V}\left(x \mid B^{(H)} ; c\right)+\dot{V}\left(x+\sigma_{n} z \mid B^{(H)} ; c\right)\right)-\frac{\sigma_{n}^{2}}{8}\left(\dot{V}\left(x+\sigma_{n} z \mid B^{(H)} ; c\right)^{2}\right. \\
& \left.-\dot{V}\left(x \mid B^{(H)} ; c\right)^{2}\right) .
\end{aligned}
$$

By Lemma A.2 from the Appendix (using $\delta=\sigma_{n} z$ ), and (28), bearing in mind that $\int_{0}^{1}(1-$ $2 t) \mathrm{d} t=0$,

$$
\begin{aligned}
& V\left(x+\sigma_{n} z \mid B^{(H)} ; c\right)-V\left(x \mid B^{(H)} ; c\right)-\frac{\sigma_{n} z}{2}\left(\dot{V}\left(x \mid B^{(H)} ; c\right)+\dot{V}\left(x+\sigma_{n} z \mid B^{(H)} ; c\right)\right) \\
& \quad=\frac{\sigma_{n}^{2} z^{2}}{2} \int_{0}^{1}(1-2 t) \ddot{V}\left(x+t \sigma_{n} z \mid B^{(H)} ; c\right) \mathrm{d} t=\frac{\sigma_{n}^{2} z^{2}}{2} \int_{0}^{1} \varphi_{c}\left(x+t \sigma_{n} z\right) B_{x+t \sigma_{n} z}^{(H)}(1-2 t) \mathrm{d} t \\
& =M_{n}\left(x, z \mid B^{(H)} ; c\right)+\Delta_{n}^{(1)}\left(x, z \mid B^{(H)} ; c\right) .
\end{aligned}
$$

On the other hand, noting that $\frac{\partial}{\partial t}\left(\dot{V}^{2}(t)\right)=2 \dot{V}(t) \ddot{V}(t)$, by the fundamental theorem of calculus

$$
\begin{aligned}
& \frac{\sigma_{n}^{2}}{8}\left(\dot{V}\left(x+\sigma_{n} z \mid B^{(H)} ; c\right)^{2}-\dot{V}\left(x \mid B^{(H)} ; c\right)^{2}\right) \\
& \quad=\frac{\sigma_{n}^{3} z}{4} \int_{0}^{1} \ddot{V}\left(x+t \sigma_{n} z \mid B^{(H)} ; c\right) \dot{V}\left(x+t \sigma_{n} z \mid B^{(H)} ; c\right) \mathrm{d} t=\Delta_{n}^{(2)}\left(x, z \mid B^{(H)} ; c\right) .
\end{aligned}
$$

Property (ii): the centred Gaussian distribution property follows from the fact that fBM is a centred Gaussian process. Moreover,

$\mathbb{E}\left[M_{n}\left(x, z \mid B^{(H)} ; c\right)^{2}\right]=\varphi_{c}(x)^{2} \sigma_{n}^{4} z^{4} \int_{0}^{1} \int_{0}^{1} \Gamma^{(H)}\left(x+t \sigma_{n} z, x+s \sigma_{n} z\right)(1-2 t)(1-2 s) \mathrm{d} t \mathrm{~d} s$.

Recall the formula for the covariance of fBM in (2) and note that all the terms that do not depend on both $t$ and $s$ must vanish when integrated with respect to $(1-2 t)(1-2 s) \mathrm{d} t \mathrm{~d} s$. Hence

$$
\mathbb{E}\left[M_{n}\left(x, z \mid B^{(H)} ; c\right)^{2}\right]=-\frac{\varphi_{c}(x)^{2}}{2}|z|^{4+2 H} \sigma_{n}^{4+2 H} \int_{0}^{1} \int_{0}^{1}|t-s|^{2 H}(1-2 t)(1-2 s) \mathrm{d} t \mathrm{~d} s .
$$

The result is now obtained by noting that the last integral equals $-\frac{H}{2+7 H+7 H^{2}+2 H^{3}}$ (nonzero and bounded for $H \in(0,1))$.

Property (iii): The random variable $\Delta_{n}^{(1)}\left(x, z \mid B^{(H)} ; c\right)$ is centred normal: this again follows from the fact that fBM is a centred Gaussian process. Also note that by the Cauchy-Schwarz inequality, and the quantified Lipschitz property for $\varphi_{c}$ described in Lemma 29(iii),

$$
\begin{aligned}
\mathbb{E}\left[\Delta_{n}^{(1)}\left(x, z \mid B^{(H)} ; c\right)^{2}\right] & \leq \frac{\sigma_{n}^{4} z^{4}}{4} \int_{0}^{1} \mathbb{E}\left[\left(B_{x+t \sigma_{n} z}^{(H)}\right)^{2}\right]\left(\varphi_{c}\left(x+t \sigma_{n} z\right)-\varphi_{c}(x)\right)^{2} \mathrm{~d} t \\
& \leq \frac{9 \sigma_{n}^{6} z^{6}}{4} c^{\frac{1}{H}} \int_{0}^{1} \mathbb{E}\left[\left(B_{x+t \sigma_{n} z}^{(H)}\right)^{2}\right] t^{2} \mathrm{~d} t \\
& \leq \frac{9}{4} c^{-\frac{1}{H}} z^{6}\left(|x|^{H}+\left|\sigma_{n} z\right|^{H}\right)^{2} \times \sigma_{n}^{6} .
\end{aligned}
$$


This yields the required control of $\iint_{\mathbb{R}^{2}}\left|\Delta_{n}^{(1)}\left(x, z \mid B^{(H)} ; c\right)\right|^{8 m} v(x, z) \mathrm{d} x \mathrm{~d} z$, using the fact that the summand $\Delta_{n}^{(1)}\left(x, z \mid B^{(H)} ; c\right)$ is centred normal while the polynomial moments of $v$ are all bounded.

For $\Delta_{n}^{(2)}\left(x, z \mid B^{(H)} ; c\right)$ consider the bound

$$
\begin{aligned}
\mathbb{E} & {\left[\Delta_{n}^{(2)}\left(x, z \mid B^{(H)} ; c\right)^{8 m}\right] } \\
& \leq \frac{\sigma_{n}^{24 m} z^{8 m}}{4^{8 m}} \int_{0}^{1} \mathbb{E}\left[\left(\dot{V}\left(x+t \sigma_{n} z \mid B^{(H)} ; c\right) \ddot{V}\left(x+t \sigma_{n} z \mid B^{(H)} ; c\right)\right)^{8 m}\right] \mathrm{d} t \\
& \leq \frac{\sigma_{n}^{24 m} z^{8 m}}{4^{8 m}} \int_{0}^{1} \mathbb{E}\left[\dot{V}\left(x+t \sigma_{n} z \mid B^{(H)} ; c\right)^{16 m}\right]^{1 / 2} \mathbb{E}\left[\ddot{V}\left(x+t \sigma_{n} z \mid B^{(H)} ; c\right)^{16 m}\right]^{1 / 2} \mathrm{~d} t .
\end{aligned}
$$

Both expectation can be bounded above with polynomials in $x$ and $z$, since they are expectations of powers of normal random variables whose means and variances can be bounded by polynomials (see Lemma 32 and equations (27), (28)).

Finally, to demonstrate the "asymptotic weak dependence" property we need to define suitable subsets of $\mathbb{R}^{4}$. The definition is based on that of (23) but using different proposal variances $\sigma_{n}$ )

$$
\mathcal{S}_{n}:=\left\{\left(x_{1}, z_{1}, x_{2}, z_{2}\right) \in \mathbb{R}^{4}:\left|x_{1}-x_{2}\right|>2 \sigma_{n}^{1 / 2}\left(\left|z_{1}\right|+\left|z_{2}\right|\right)\right\} .
$$

LEMMA 34. Assumption 15.(D) is satisfied:

(i) $\int_{\mathcal{S}_{n}^{c}} v\left(x_{1}, z_{1}\right) v\left(x_{2}, z_{2}\right) \mathrm{d} x_{1} \mathrm{~d} z_{1} \mathrm{~d} x_{2} \mathrm{~d} z_{2} \precsim \sigma_{n}^{1 / 2}$.

(ii) For any $\left(x_{1}, x_{2}, z_{1}, z_{2}\right) \in \mathcal{S}_{n}$ we have

$$
\begin{aligned}
\left|\mathbb{E}\left[M_{n}\left(x_{1}, z_{1} \mid B^{(H)} ; c\right) M_{n}\left(x_{2}, z_{2} \mid B^{(H)} ; c\right)\right]\right| & \leq \frac{H|2 H-1|}{2^{2-2 H}}\left|z_{1}\right|^{2+H}\left|z_{2}\right|^{2+H} \sigma_{n}^{5+H} \\
& \precsim\left|z_{1}\right|^{2+H}\left|z_{2}\right|^{2+H} \sigma_{n}^{2 \beta+\gamma} .
\end{aligned}
$$

(iii) There exists a polynomial $g_{2}\left(x_{1}, z_{1}, x_{2}\right)$ such that

$$
\begin{aligned}
\left|\mathbb{E}\left[M_{n}\left(x_{1}, z_{1} \mid B^{(H)} ; c\right) K\left(x_{2} \mid B^{(H)} ; c\right)\right]\right| & \leq g_{2}\left(x_{1}, z_{1}, x_{2}\right) \sigma_{n}^{2+H+\min (H, 1-H)} \\
& =g_{2}\left(x_{1}, z_{1}, x_{2}\right) \sigma_{n}^{\beta+\gamma} .
\end{aligned}
$$

Proof. Property (i) follows by applying Lemma A.3 in the Appendix, using the sequence $a_{n}=\sigma_{n}^{1 / 2}$.

Property (ii): Using the formula (2) for the covariance function of fBM,

$$
\begin{aligned}
\mathbb{E} & {\left[M_{n}\left(x_{1}, z_{1} \mid B^{(H)} ; c\right) M_{n}\left(x_{2}, z_{2} \mid B^{(H)} ; c\right)\right] } \\
& =z_{1}^{2} z_{2}^{2} \sigma_{n}^{4} \varphi_{c}\left(x_{1}\right) \varphi_{c}\left(x_{2}\right) \int_{0}^{1} \int_{0}^{1} \Gamma^{(H)}\left(x_{1}+t \sigma_{n} z_{1}, x_{2}+s \sigma_{n} z_{2}\right)(1-2 t)(1-2 s) \mathrm{d} t \mathrm{~d} s .
\end{aligned}
$$

Again, all terms not depending on both $t$ and $s$ vanish when integrated with respect to (1 $2 t)(1-2 s) \mathrm{d} t \mathrm{~d} s$. Hence, in the expression above we can swap $\Gamma^{(H)}\left(x_{1}+t \sigma_{n} z_{1}, x_{2}+s \sigma_{n} z_{2}\right)$ for

$-\frac{1}{2}\left(\left|x_{1}-x_{2}\right|^{2 H}-\left|x_{1}-x_{2}+\sigma_{n} t z_{1}\right|^{2 H}-\left|x_{1}-x_{2}-\sigma_{n} s z_{2}\right|^{2 H}+\left|x_{1}-x_{2}+\sigma_{n}\left(t z_{1}-s z_{2}\right)\right|^{2 H}\right)$.

Using Lemma A.3(ii) from the Appendix with $u=\sigma_{n}^{1 / 2} t, v=\sigma_{n}^{1 / 2} s$ (assuming $n$ large enough that $\sigma_{n} \leq 1$ ), and the details of construction of the set $\mathcal{S}_{n}$ in (33), if it is the case that 
$\left(x_{1}, z_{1}, x_{2}, z_{2}\right) \in \mathcal{S}_{n}$ then it must be the case that $\left|x_{1}-x_{2}+\sigma_{n}\left(t z_{1}-s z_{2}\right)\right|>\frac{\left|x_{1}-x_{2}\right|}{2}>0$ and $\left|\sigma_{n}\left(t z_{1}-s z_{2}\right)\right|<\frac{\left|x_{1}-x_{2}\right|}{2}$ for each $t, s \in(0,1)$. So $x_{1}-x_{2}+\sigma_{n}\left(t z_{1}-s z_{2}\right)$ is at least a positive distance away from zero and of the same sign for all $t, s \in[0,1]$. Hence, since the function $x \mapsto|x|^{2 H}$ is smooth away from zero, Lemma A.1 from the Appendix implies that

$$
\begin{aligned}
& \Gamma^{(H)}\left(x_{1}+t \sigma_{n} z_{1}, x_{2}+s \sigma_{n} z_{2}\right) \\
& \quad=H(2 H-1) t s \sigma_{n}^{2} z_{1} z_{2} \int_{0}^{1} \int_{0}^{1}\left|x_{1}-x_{2}+\sigma_{n}\left(u t z_{1}-v s z_{2}\right)\right|^{2 H-2} \mathrm{~d} u \mathrm{~d} v .
\end{aligned}
$$

Therefore, by Lemma A.3(ii) from the Appendix, and construction of $\mathcal{S}_{n}$ in (33):

$$
\begin{aligned}
\mid \mathbb{E} & {\left[M_{n}\left(x_{1}, z_{1} \mid B^{(H)} ; c\right) M_{n}\left(x_{2}, z_{2} \mid B^{(H)} ; c\right)\right] \mid } \\
& \leq H|2 H-1|\left|z_{1} z_{2}\right|^{3}\left|\frac{x_{1}-x_{2}}{2}\right|^{2 H-2} \sigma_{n}^{6} \\
& =H|2 H-1|\left|z_{1}\right|^{2+H}\left|z_{2}\right|^{2+H}\left(\frac{2 \sqrt{\left|z_{1}\right|\left|z_{2}\right|}}{\left|x_{1}-x_{2}\right|}\right)^{2-2 H} \sigma_{n}^{6} \\
& \leq H|2 H-1|\left|z_{1}\right|^{2+H}\left|z_{2}\right|^{2+H}\left(\frac{\left|z_{1}\right|+\left|z_{2}\right|}{\left|x_{1}-x_{2}\right|}\right)^{2-2 H} \sigma_{n}^{6} \\
& \leq \frac{H|2 H-1|}{2^{2-2 H}}\left|z_{1}\right|^{2+H}\left|z_{2}\right|^{2+H} \sigma_{n}^{5+H} .
\end{aligned}
$$

Property (iii): We now need to distinguish between the cases $H \lesseqgtr \frac{1}{2}$. First, consider the case $H \leq 1 / 2$, so that $2 H \leq 1$ Observe that by (25) and (32)

$$
\begin{aligned}
\mathbb{E}[ & \left.M_{n}\left(x_{1}, z_{1} \mid B^{(H)} ; c\right) K\left(x_{2} \mid B^{(H)} ; c\right)\right] \\
= & \sigma_{n}^{2} z_{1}^{2} x_{2}^{2} \int_{0}^{1} \int_{0}^{1} \varphi_{c}\left(x_{1}\right) \varphi_{c}\left(s x_{2}\right) \Gamma^{(H)}\left(x_{1}+t \sigma_{n} z_{1}, s x_{2}\right)(1-2 t)(1-s) \mathrm{d} t \mathrm{~d} s \\
= & \frac{1}{2} \sigma_{n}^{2} z_{1}^{2} x_{2}^{2} \int_{0}^{1} \int_{0}^{1} \varphi_{c}\left(x_{1}\right) \varphi_{c}\left(s x_{2}\right) \\
& \times\left(\left|x_{1}+t \sigma_{n} z_{1}\right|^{2 H}-\left|x_{1}\right|^{2 H}-\left|x_{1}-s x_{2}+t \sigma_{n} z_{1}\right|^{2 H}+\left|x_{1}-s x_{2}\right|^{2 H}\right) \\
& \times(1-2 t)(1-s) \mathrm{d} t \mathrm{~d} s .
\end{aligned}
$$

The second equality holds since the difference of integrands does not depend on $t$ and thus integrates to zero. Since $2 H \leq 1$ and ||$x_{1}+\left.t \sigma_{n} z_{1}\right|^{2 H}-\left.\left|x_{1}\right|^{2 H}\left|\leq \sigma_{n}^{2 H}\right| z_{1}\right|^{2 H}$ (similarly for the other term), we obtain $\left|\mathbb{E}\left[M_{n}\left(x_{1}, z_{1} \mid B^{(H)} ; c\right) K\left(x_{2} \mid B^{(H)} ; c\right)\right]\right| \leq \sigma_{n}^{2+2 H}\left|z_{1}\right|^{2+2 H} x_{2}^{2}$.

Second, consider the case $H>1 / 2$, so that $0<2 H-1<1$ and the function $x \mapsto|x|^{2 H}$ has a continuous derivative $x \mapsto 2 H \operatorname{sign}(x)|x|^{2 H-1}$. The Fundamental theorem of calculus then implies

$$
\begin{aligned}
|| x_{1}+\left.t \sigma_{n} z_{1}\right|^{2 H}-\left|x_{1}\right|^{2 H} \mid & \leq 2 H\left|t \sigma_{n} z_{1} \int_{0}^{1} \operatorname{sign}\left(x_{1}+u t \sigma_{n} z_{1}\right)\right| x_{1}+\left.u t \sigma_{n} z_{1}\right|^{2 H-1} \mathrm{~d} u \mid \\
& \leq 2 H \sigma_{n}\left|z_{1}\right| \int_{0}^{1}\left|x_{1}+u t \sigma_{n} z_{1}\right|^{2 H-1} \mathrm{~d} u \\
& \leq 2 H \sigma_{n}\left|z_{1}\right|\left(\left|x_{1}\right|^{2 H-1}+\sigma_{n}^{2 H-1}\left|z_{1}\right|^{2 H-1}\right) .
\end{aligned}
$$


An analogous bound holds for ||$x_{1}-s x_{2}+\left.t \sigma_{n} z_{1}\right|^{2 H}-\left|x_{1}-s x_{2}\right|^{2 H} \mid$ and together

$$
\begin{aligned}
\mid \mathbb{E}[ & \left.M_{n}\left(x_{1}, z_{1} \mid B^{(H)} ; c\right) K\left(x_{2} \mid B^{(H)} ; c\right)\right] \mid \\
& \leq H\left|z_{1}\right|^{3} x_{2}^{2}\left(2\left|x_{1}\right|^{2 H-1}+\left|x_{2}\right|+2 \sigma_{n}^{2 H-1}\left|z_{1}\right|^{2 H-1}\right) \times \sigma_{n}^{3} .
\end{aligned}
$$

Now we are in a position to prove Theorem 2.

Proof of TheOREM 2. Lemmas 30, 32, 33 and 34 together show that the anomalous scaling framework 15 holds for the MALA algorithm as described at the head of this section and as stipulated by Theorem 2. Consequently Theorem 2 is a direct consequence of Theorem 24.

8. Discussion. In this concluding section we discuss how our results relate to considerations of expected squared jump distance, further research possibilities, and some practical considerations concerning how our results might relate to questions of practical Markov chain Monte Carlo.

8.1. Expected squared jump distance. In the setting of either Theorem 1 or Theorem 2, in particular when the $X_{i} \sim \pi\left(\cdot \mid B^{(H)}\right)$ are conditionally independent and identically distributed and $\sigma_{n}=\ell n^{-\frac{1}{2 \beta}}$, and given a positive sequence $\vartheta_{1}, \vartheta_{2}, \ldots$ decaying to zero, we define proposals $Y_{i}^{(n), \vartheta} \sim q_{n}^{\vartheta}\left(X_{i}, d y\right) \sim N\left(X_{i}, \vartheta_{n}^{2} \cdot I_{n}\right)$ in the RWM case and $Y_{i}^{(n), \vartheta} \sim q_{n}^{\vartheta}\left(X_{i}, d y\right) \sim$ $N\left(X_{i}+\frac{\vartheta_{n}^{2}}{2} \dot{V}\left(X_{i} \mid B^{(H)} ; c\right), \vartheta_{n}^{2} \cdot I_{n}\right)$ in the MALA case. We also define random variables which measure the growth/decay rate of the expected squared jump distance (ESJD) relative to $\sigma_{n}^{2}$ for different scalings of proposal variance; these are conditional expectations given $B^{(H)}$ as follows:

$$
\operatorname{ESJD}_{n}\left(B^{(H)}, \vartheta_{n}\right)=n^{\frac{1}{\beta}} \times \mathbb{E}\left[\left(Y_{1}^{(n), \vartheta}-X_{1}\right)^{2}(1 \wedge \exp )\left(\sum_{i=1}^{n} \rho\left(X_{i}, Y_{i}^{(n), \vartheta}\right)\right) \mid B^{(H)}\right]
$$

From either Theorem 1 or Theorem 2 we can deduce that almost surely (when conditioned on $B^{(H)}$ )

$$
\operatorname{ESJD}_{n}\left(B^{(H)}, \sigma_{n}\right) \stackrel{B^{(H)} \text { a.s. }}{\longrightarrow} W(\ell):=2 \ell^{2} \Phi\left(-\frac{\ell^{\beta} \theta}{2}\right)
$$

for an appropriate positive random variable $\theta$ that is $B^{(H)}$-measurable (see Theorem 1 or Theorem 2 and Roberts, Gelman and Gilks [29], Proposition 2.4). This can be shown by adopting the method of proof of Corollary 18 in [35], where we realise all the $X_{i}, Y_{i}^{(n)}$ on the same probability space and use the tower property.

We seek to show that the rate of $\operatorname{ESJD}_{n}\left(B^{(H)}, \vartheta_{n}\right)$ is optimal when $\vartheta_{n} \sim \sigma_{n}$. More precisely, we must show that the rate converges to zero almost surely for $\vartheta_{n}$ with decay rate differing asymptotically from the decay rate of $\sigma_{n}$. If $\frac{\vartheta_{n}}{\sigma_{n}} \rightarrow 0$, it is straightforward to show $\operatorname{ESJD}_{n}\left(B^{(H)}, \vartheta_{n}\right) \rightarrow 0$ almost surely. Indeed, we simply note the acceptance rate is bounded above (by 1) and argue that

$$
\begin{aligned}
& \limsup _{n \rightarrow \infty} n^{\frac{1}{\beta}} \times \mathbb{E}\left[\left(Y_{1}^{(n), \vartheta}-X_{1}\right)^{2}(1 \wedge \exp )\left(\sum_{i=1}^{n} \rho\left(X_{i}, Y_{i}^{(n), \vartheta}\right)\right) \mid B^{(H)}\right] \\
& \precsim \lim _{n \rightarrow \infty} \sigma_{n}^{-2} \times \mathbb{E}\left[\left(Y_{1}^{(n), \vartheta}-X_{1}\right)^{2} \mid B^{(H)}\right]=\lim _{n \rightarrow \infty} \frac{\vartheta_{n}^{2}}{\sigma_{n}^{2}} \rightarrow 0 .
\end{aligned}
$$


Unfortunately, when $\frac{\vartheta_{n}}{\sigma_{n}} \rightarrow \infty$ we can only show convergence in probability

$$
\operatorname{ESJD}_{n}\left(B^{(H)}, \vartheta_{n}\right) \stackrel{\mathrm{P}}{\rightarrow} 0 .
$$

The reason is that, even though Lemmas 16, 17 and 18, as well as Lemma 22, all remain valid even if we use proposal variances $\vartheta_{n}^{2}$ instead of $\sigma_{n}^{2}$ (we only require $\vartheta_{n} \rightarrow 0$ ), we cannot recover a result analogous to the Borel-Cantelli argument of Proposition 19 for arbitrary decay rates of proposal variances, only for rates $\vartheta_{n}$ "close" to $\sigma_{n}$. In effect, it can thus be shown that the decay rate is "locally optimal", but not necessarily "globally optimal". This is an intrinsic issue for the anomalous scaling framework method 15 described in Section 5: even with better bounds or a different random environment construction it will always be possible to construct decay rates $\vartheta_{n}$ that are slow enough to ensure that Borel-Cantelli arguments fail, as a result of certain series not being summable.

The need to restrict to convergence in probability suggests that the setting of [29] will not apply to the setting of Theorems 1 and 2, since of course subsequence arguments will then imply existence of subsequences of increasing dimension along which classical scaling is not optimal. It seems unlikely that almost sure convergence would ever not hold, but a proof of this in the case $\frac{\vartheta_{n}}{\sigma_{n}} \rightarrow \infty$ would have to deal with varying and very different decay rates of the proposal variance $\vartheta_{n}^{2}$.

Nevertheless, for any $\frac{\vartheta_{n}}{\sigma_{n}} \rightarrow \infty$ we can recover weaker versions of Corollaries 21 and 23:

$$
\vartheta_{n}^{-2 \beta} \mathbb{E}\left[\rho_{n}^{2}\left(X, Y_{i}^{(n), \vartheta} \mid B^{(H)}\right) \mid B^{(H)}\right] \stackrel{P}{\rightarrow} \theta
$$

and

$$
\vartheta_{n}^{-2 \beta} \mathbb{E}\left[\rho_{n}^{2}\left(X, Y^{(n), \vartheta} \mid B^{(H)}\right) 1_{\rho_{n}^{2}\left(X, Y^{(n), \vartheta}\right)>\vartheta_{n}^{\beta}} \mid B^{(H)}\right] \stackrel{\mathrm{P}}{\rightarrow} 0 .
$$

This is enough to establish our objective, equation (35). And for this it suffices to show that the almost sure versions of (36) and (37) imply $\operatorname{ESJD}_{n}\left(B^{(H)}, \vartheta_{n}\right) \rightarrow 0$ almost surely. Proof of convergence in probability then follows using the celebrated characterisation of convergence in probability as holding whenever subsequences all have almost surely convergent sub-subsequences.

LEMMA 35. Assume $\frac{\vartheta_{n}}{\sigma_{n}} \rightarrow \infty$. Almost sure versions of (36) and (37) imply $\operatorname{ESJD}_{n}\left(B^{(H)}\right.$, $\left.\vartheta_{n}\right) \rightarrow 0$ almost surely.

PROOF. Theorem 7 ("mean plus half-variance is asymptotically negligible") together with almost sure versions of (36) and (37) implies

$$
\vartheta_{n}^{-2 \beta}\left(\mathbb{E}\left[\rho_{n}\left(X, Y^{(n), \vartheta} \mid B^{(H)}\right)\right]+\frac{1}{2} \operatorname{Var}\left[\rho_{n}\left(X, Y^{(n), \vartheta} \mid B^{(H)}\right) \mid B^{(H)}\right]\right) \stackrel{\text { a.s. }}{\longrightarrow} 0 .
$$

Write $\rho_{i}^{(n), \vartheta}=\rho_{n}\left(X_{i}, Y_{i}^{(n), \vartheta} \mid B^{(H)}\right)$. The $\rho_{i}^{(n), \vartheta}$ are independent given $B^{(H)}$, and so (38) yields

$$
\frac{1}{n \vartheta_{n}^{2 \beta}} \operatorname{Var}\left[\sum_{i=1}^{n} \rho_{i}^{(n), \vartheta} \mid B^{(H)}\right]=\vartheta_{n}^{-2 \beta} \operatorname{Var}\left[\rho_{1}^{(n), \vartheta} \mid B^{(H)}\right] \stackrel{\text { a.s. }}{\longrightarrow} \theta
$$

and

$$
\frac{1}{n \vartheta_{n}^{2 \beta}} \mathbb{E}\left[\sum_{i=1}^{n} \rho_{i}^{(n), \vartheta} \mid B^{(H)}\right]=\vartheta_{n}^{-2 \beta} \mathbb{E}\left[\rho_{1}^{(n), \vartheta} \mid B^{(H)}\right] \stackrel{\text { a.s. }}{\longrightarrow}-\frac{\theta}{2}
$$


Consequently, for all large enough $n$,

$$
\begin{aligned}
\mathbb{P} & {\left[\sum_{i=1}^{n} \rho_{i}^{(n), \vartheta}>-\frac{1}{4} \operatorname{Var}\left[\sum_{i=1}^{n} \rho_{i}^{(n), \vartheta} \mid B^{(H)}\right] \mid B^{(H)}\right] } \\
& \leq \mathbb{P}\left[\left|\sum_{i=1}^{n}\left(\rho_{i}^{(n), \vartheta}-\mathbb{E}\left[\rho_{i}^{(n), \vartheta} \mid B^{(H)}\right]\right)\right|>\frac{1}{5} \operatorname{Var}\left[\sum_{i=1}^{n} \rho_{n, i} \mid B^{(H)}\right] \mid B^{(H)}\right] \\
& =\mathbb{P}\left[\left(\sum_{i=1}^{n}\left(\rho_{i}^{(n), \vartheta}-\mathbb{E}\left[\rho_{i}^{(n), \vartheta} \mid B^{(H)}\right]\right)\right)^{4 m}>\frac{1}{5^{4 m}} \operatorname{Var}\left[\sum_{i=1}^{n} \rho_{n, i} \mid B^{(H)}\right]^{4 m} \mid B^{(H)}\right],
\end{aligned}
$$

for $m$ chosen to satisfy the requirements of Framework 15. From the proof of Lemma 22 we may conclude

$$
\mathbb{E}\left[\left(\rho_{i}^{(n), \vartheta}-\mathbb{E}\left[\rho_{i}^{(n), \vartheta} \mid B^{(H)}\right]\right)^{4 m} \mid B^{(H)}\right] \leq \mathbb{E}\left[\left(\rho_{i}^{(n), \vartheta}\right)^{4 m} \mid B^{(H)}\right] \precsim \vartheta_{n}^{4 m \beta} .
$$

Following the argument of Mijatović and Vogrinc ([22], Proposition 26), given centred IID random variables $A_{1}, \ldots, A_{n} \sim A$ satisfying $\mathbb{E}\left[A^{4 m}\right]<\infty$ we have

$$
\begin{aligned}
& \left.\mathbb{E}\left[\left(A_{1}+\cdots+A_{n}\right)^{4 m}\right]=\sum_{\substack{s_{1}+\cdots+s_{n}=4 m \\
s_{i} \in\{0,2,3, \ldots\}}} \cdots \sum_{1}^{s_{1}} \cdots A_{n}^{s_{n}}\right] \\
& \leq \sum_{\substack{s_{1}+\cdots+s_{n}=4 m \\
s_{i} \in\{0,2,3, \ldots\}}} \cdots \sum_{\substack{s_{1} \\
s_{1}}} \mathbb{E}\left[\mid A_{1}\right] \mathbb{E}\left[\left|A_{n}\right|^{s_{n}}\right] \\
& \leq \sum_{\substack{s_{1}+\cdots+s_{n}=4 m \\
s_{i} \in\{0,2,3, \ldots\}}} \cdots \sum_{\substack{s_{1} \\
s^{\prime}}} \mathbb{E}\left[\left|A_{1}\right|^{4 m}\right]^{\frac{s_{1}}{4 m}} \cdots \mathbb{E}\left[\left|A_{n}\right|^{4 m}\right]^{\frac{s_{n}}{4 m}} \\
& =\left(\sum_{\substack{s_{1}+\cdots+s_{n}=4 m \\
s_{i} \in\{0,2,3, \ldots\}}} \cdots \sum_{t_{1}+\cdots+t_{n}=2 m} 1\right) \mathbb{E}\left[\left|A_{1}\right|^{4 m}\right] \leq 3^{2 m}\left(\sum_{t^{\prime}} \cdots \sum_{1} 1\right) \mathbb{E}\left[\left|A_{1}\right|^{4 m}\right] \\
& =3^{2 m} n^{2 m} \mathbb{E}\left[\left|A_{1}\right|^{4 m}\right] \text {. }
\end{aligned}
$$

Here the first equality holds because all the terms containing exactly one copy of any of the $A_{i}$ vanish due to $A_{i}$ being centred and independent; the third inequality arises from Jensen's inequality; the fourth inequality is obtained by mapping each tuple $\left(s_{1}, \ldots, s_{n}\right)$ to $\left(t_{1}, \ldots, t_{n}\right)$ by dividing $s_{i}$ by 2 if $s_{i}$ is even, otherwise alternately increasing or decreasing $s_{i}$ by 1 then dividing by 2 . Each resulting tuple $\left(t_{1}, \ldots, t_{n}\right)$ sums to $2 m$ and derives from no more than $3^{2 m}$ of the $\left(s_{1}, \ldots, s_{n}\right)$ tuples.

Using Markov's inequality on (40) and then using (41) together with (42) shows

$$
\begin{gathered}
\mathbb{P}\left[\sum_{i=1}^{n} \rho_{i}^{(n), \vartheta}>-\frac{1}{4} \operatorname{Var}\left[\sum_{i=1}^{n} \rho_{i}^{(n), \vartheta} \mid B^{(H)}\right] \mid B^{(H)}\right] \\
\precsim n^{2 m} \times \frac{\vartheta_{n}^{4 m \beta}}{\operatorname{Var}\left[\sum_{i=1}^{n} \rho_{i}^{(n), \vartheta} \mid B^{(H)}\right]^{4 m}} \\
\precsim n^{2 m} \times \frac{\vartheta_{n}^{4 m \beta}}{n^{4 m} \vartheta_{n}^{8 m \beta} \theta^{4 m}}=\frac{\sigma_{n}^{4 m}}{\vartheta_{n}^{4 m \beta} \ell^{4 m} \theta^{4 m}},
\end{gathered}
$$

where we have used (39) (changing the constant) for the second bound and have used $n \sigma_{n}^{2 \beta}=$ $\ell^{2 \beta}$ (as stipulated in Framework 15) for the final equality. 
In both the RWM and MALA case we have

$$
\mathbb{E}\left[\left(Y_{1}^{(n), \vartheta}-X_{1}\right)^{4} \mid B^{(H)}\right] \precsim \vartheta_{n}^{4} .
$$

The RWM case is trivial, while in the MALA case

$$
\mathbb{E}\left[\left(Y_{1}^{(n), \vartheta}-X_{1}\right)^{4} \mid B^{(H)}\right]=\vartheta_{n}^{4} \iint_{\mathbb{R}^{2}}\left(z+\frac{\vartheta_{n}}{2} \dot{V}\left(x \mid B^{(H)} ; c\right)\right)^{4} \pi\left(x \mid B^{(H)} ; c\right) \nu_{1}(z) \mathrm{d} x \mathrm{~d} z
$$

equals $3 \vartheta_{n}^{4}$ plus a sum of higher powers of $\vartheta_{n}$ multiplied by random variables that depend only on $B^{(H)}$ and are almost surely finite by Assumption 15.(A) and Lemma 31(i).

So now consider: under the constraint $\sum_{i=1}^{n} \rho_{i}^{(n), \vartheta} \leq-\frac{1}{4} \operatorname{Var}\left[\sum_{i=1}^{n} \rho_{i}^{(n), \vartheta} \mid B^{(H)}\right]$ the resulting upper bound on the acceptance rate leads, together with (44) and (39), to

$$
\begin{aligned}
n^{\frac{1}{\beta}} \times & \mathbb{E}\left[\left(Y_{1}^{(n), \vartheta}-X_{1}\right)^{2}(1 \wedge \exp )\left(\sum_{i=1}^{n} \rho\left(X_{i}, Y_{i}^{(n), \vartheta} \mid B^{(H)}\right)\right) ;\right. \\
& \left.\sum_{i=1}^{n} \rho_{i}^{(n), \vartheta} \leq-\frac{1}{4} \operatorname{Var}\left[\sum_{i=1}^{n} \rho_{i}^{(n), \vartheta} \mid B^{(H)}\right] \mid B^{(H)}\right] \\
\precsim & \frac{\vartheta_{n}^{2}}{\sigma_{n}^{2}} \exp \left(-\frac{1}{4} \operatorname{Var}\left[\sum_{i=1}^{n} \rho_{i}^{(n), \vartheta} \mid B^{(H)}\right]\right) \precsim \frac{\vartheta_{n}^{2}}{\sigma_{n}^{2}} \exp \left(-\frac{\ell^{2 \beta} \theta}{3} \frac{\vartheta_{n}^{2 \beta}}{\sigma_{n}^{2 \beta}}\right) \stackrel{\text { a.s. }}{\longrightarrow} 0 .
\end{aligned}
$$

Here we reduce the denominator in the final exponent from 4 to 3 to control fluctuations in the limit for the scaled variance expressed by equation (39).

Alternatively, under the constraint $\sum_{i=1}^{n} \rho_{i}^{(n), \vartheta}>-\frac{1}{4} \operatorname{Var}\left[\sum_{i=1}^{n} \rho_{i}^{(n), \vartheta} \mid B^{(H)}\right]$ we can apply the Cauchy-Schwarz inequality together with the limits (43) and (44). Using $n \sigma_{n}^{2 \beta}=\ell^{2 \beta}$ again,

$$
\begin{aligned}
n^{\frac{1}{\beta}} \times & \mathbb{E}\left[\left(Y_{1}^{(n), \vartheta}-X_{1}\right)^{2}(1 \wedge \exp )\left(\sum_{i=1}^{n} \rho\left(X_{i}, Y_{i}^{(n), \vartheta} \mid B^{(H)}\right)\right) ;\right. \\
& \left.\sum_{i=1}^{n} \rho_{i}^{(n), \vartheta}>-\frac{1}{4} \operatorname{Var}\left[\sum_{i=1}^{n} \rho_{i}^{(n), \vartheta} \mid B^{(H)}\right] \mid B^{(H)}\right] \\
\precsim & \sigma_{n}^{-2} \times \mathbb{E}\left[\left(Y_{1}^{(n), \vartheta}-X_{1}\right)^{4} \mid B^{(H)}\right]^{1 / 2} \\
& \times \mathbb{P}\left[\sum_{i=1}^{n} \rho_{i}^{(n), \vartheta}>-\frac{1}{4} \operatorname{Var}\left[\sum_{i=1}^{n} \rho_{i}^{(n), \vartheta} \mid B^{(H)}\right] \mid B^{(H)}\right]^{1 / 2} \\
\precsim & \frac{\vartheta_{n}^{2}}{\sigma_{n}^{2}} \times \frac{1}{\ell^{2 m \beta} \theta^{2 m}} \times \frac{\sigma_{n}^{2 m \beta}}{\vartheta_{n}^{2 m \beta}}=\frac{1}{\ell^{2 m \beta} \theta^{2 m}} \times \frac{\sigma_{n}^{2 m \beta-2}}{\vartheta_{n}^{2 m \beta-2}}
\end{aligned}
$$

which almost surely converges to zero provided $m \beta>1$, since $\frac{\vartheta_{n}}{\sigma_{n}} \rightarrow \infty$. In the case of MALA $\beta=2+H$ we need only use $m=1$; however in the RWM case $\beta=H$, so we need to choose $m$ to be sufficiently large (recall that all polynomial moments of $\rho_{i}^{(n), \vartheta}$ are finite). Together the above bounds give

$$
\operatorname{ESJD}\left(B^{(H)}, \vartheta_{n}\right) \stackrel{\text { a.s. }}{\rightarrow} 0 .
$$

Accepting that $\sigma_{n}^{2}$ is the optimal decay rate for proposal variances, we turn our attention to choosing the $\ell$ that maximises the ESJD, equivalently (as it will turn out) determining the 
optimal average acceptance rate. Revisiting $(34), W(\ell)$ takes the form

$$
W(\ell)=2 \ell^{2} \times \Phi\left(-\frac{\sigma(\ell)}{2}\right)=2 \ell^{2} \times \Phi\left(-\frac{\ell^{\beta} \theta}{2}\right)
$$

for $\sigma(\ell)$ as in Theorems 1 and 2, for $\theta$ a positive constant that depends only on $B^{(H)}$ and for $\Phi$ the cumulative distribution function of a standard normal random variable. (To obtain the first equality integrate $1 \wedge \exp$ with respect to the standard normal density as in [29], Proposition 2.4.)

Clearly, $W(\ell)$ is smooth, positive and converges to zero when either $\ell \rightarrow 0$ or $\ell \rightarrow \infty$. Its maximum is therefore achieved at a stationary point. Taking derivatives and substituting $a=\frac{\ell^{2 \beta} \theta}{2}$ leads to the equation

$$
2 \Phi(-a)-\beta a \varphi(-a)=0,
$$

where $\varphi$ is the standard normal density function. This equation has a unique solution for positive $a$ (because $a \mapsto a \frac{\varphi(-a)}{\Phi(-a)}$ is strictly increasing) and the average acceptance rate at the optimal $a$ (and optimal $\ell$ ) is then given by $2 \Phi(-a)=2 \Phi\left(-\frac{\ell^{\beta} \theta}{2}\right)$. We can solve the above equation numerically for various $\beta(H)=H$ for RWM and $\beta(H)=2+H$ for MALA to obtain the associated optimal acceptance rates. The numerical results for both RWM and MALA are presented in Figure 1. Since the left and right side of Figure 1 are both obtained by numerically solving the same equation over different disjoint ranges of parameter $\beta$, it is tempting to speculate that when using MALA for targets of smoothness class between 1 and 2 the optimal acceptance rates interpolate between the plots of Figure 1 and attain values between $23 \%$ and $45 \%$.

\subsection{Further work and open questions.}

(a) The following question remains: does there exist a "Langevin diffusion" limit result analogous to the main weak convergence results in [29] (see (1)) and [30]? We do not pursue this question here as it does not fundamentally contribute to the force of the counterexamples. Note that it is not a trivial question as the gradient of the marginal does not exist in the RWM case. Hence, we can talk about an associated Langevin diffusion in terms of its Dirichlet form but not as a strong solution of an SDE with Lipschitz coefficients.

However, we expect soon to be able to obtain a positive answer, namely that it will prove possible to show that RWM and MALA chains (with targets and proposals as specified respectively in Theorems 1 and 2) converge weakly

$$
X_{\left\lfloor n^{1 / H} \cdot t\right\rfloor, 1}^{\mathrm{RWM},(n)} \stackrel{w}{\rightarrow} U_{t} \quad \text { and } \quad X_{\left\lfloor n^{1 /(2+H) \cdot t\rfloor, 1}\right.}^{\mathrm{MALA},(n)} \stackrel{w}{\rightarrow} U_{t}
$$

to a "Langevin diffusion" $U$ with a speed parameter

$$
h(\ell)=W(\ell)=2 \ell^{2} \times \Phi\left(-\frac{\sigma(\ell)}{2}\right)=2 \ell^{2} \times \Phi\left(-\frac{\ell^{\beta} \theta}{2}\right)
$$

where $\sigma(\ell)$ and $\theta$ are compatible with Section 8.1 above and determined by Theorems 1 and 2. (Of course, this also leads to the optimal acceptance rate heuristics as noted above at the end of Section 8.1.)

To be specific, we plan to adapt the Dirichlet form methodology of Zanella, Bédard and Kendall [35] to deliver these anomalous scaling results at the level of weak convergence. With the same methodology we also expect to recover the MALA results of Roberts and Rosenthal [30] with smoothness assumptions only slightly stronger than $\mathcal{C}^{3}(\mathbb{R})$. We shall report on this more general picture as part of an upcoming review paper that will demonstrate the use of Dirichlet forms to provide a general framework for proving various results on optimal MCMC scaling. 
(b) We note an obvious question that expands this line of thought, namely, how much the random environment approach to optimal scaling can be generalised and can anything be gained from doing so? For example, is it feasible to take a different realisation of $B^{(H)}$ in each coordinate of the product structure? Can the realisations of the random environment be sampled for each $n$ ? Can we instead deal with perturbing a deterministic product target by a Gaussian process indexed by $\mathbb{R}^{n}$ ? These questions are challenging but attractive for further study, since this line of thinking offers a new way of expanding optimal scaling results beyond the product case.

A possibly fruitful extension of the random environment approach might lie in the investigation of MCMC smoothness requirements for boundaries. We also note that random environments could be used to generate further kinds of counterexamples in MCMC (not connected to roughness of the target) or to study properties of MCMC methods when averaged over a random environment in contexts where actual properties resist direct investigation.

(c) Despite presenting only very particular examples we conjecture that the type of anomalous MCMC behaviour presented here happens in substantial generality and may indeed be typical when dealing with rough targets. One possible approach to support this conjecture would be to explore the actual analytical properties provided by the random environment when arguing for anomalous scaling results. In particular it would be most interesting if one could establish that anomalous scaling was typical within a certain class of functions in the sense of Baire category: compare the development of sparsity results for contours, moving from Kendall [16] to Kendall [17].

(d) Another line of work that may be relevant is presented in Neal, Roberts and Kong Yuen [23]. They also deal with badly behaved targets for RWM. They consider discontinuous product targets, such that the one dimensional marginals are $\mathcal{C}^{2}$ on $[0,1]$ and zero outside. They establish optimal scaling rate for the proposal variance $n^{-2}$ for dimension $n$, coinciding with the case $H=\frac{1}{2}$ in our setting. However optimal acceptance rates differ because of different constructions of the Langevin diffusion. Is there a link between the behaviours captured in their paper and in ours? It is natural to wonder whether both phenomena could be explained within a common framework.

(e) Understanding the behaviour of MCMC methods not initiated in stationarity is very important for practical applications. Theoretically this has been studied together with optimal scaling results for instance in Christensen, Roberts and Rosenthal [4], Jourdain, Lelievre and Miasojedow [13, 14], Kuntz, Ottobre and Stuart [18], Kuntz et al. [19]. It is demonstrated that (for MALA) not starting in stationarity can worsen the optimal scaling rate for some initial configurations, particularly some chosen close to the mode of the target.

We did not theoretically study this question in our setting. However, numerically the RWM chains on rough targets introduced in Theorem 1 seem to behave as predicted by the theorem despite not initiated in stationarity (see Section 8.3(a)).

Appropriate modification of the random environment approach could potentially be used to identify further examples of MCMC in a nonstationary phase exhibiting worse than expected scaling behaviour.

\subsection{Heuristics for use in applications.}

(a) Let us first numerically verify what theoretical results predict. Consider an $n=200$ dimensional RWM example with $H=\frac{1}{2}$. We pre-simulate a Brownian motion path at a very fine resolution (at $2 \cdot 10^{5}$ equally spaced points between -9 and 9 ) and use linear interpolation in between grid points to evaluate the target. All computation below was done with the same fixed pre-simulated Brownian motion path. Additionally, we start the RWM according to a standard normal and use a large burn in to achieve approximate stationarity. 
TABLE 1

Average acceptance rates $\alpha$ and ESJD for different values of $\ell$ for a RWM example with rough target

\begin{tabular}{lcccccc}
\hline & \multicolumn{7}{c}{$\ell$} \\
\cline { 2 - 7 } & 5.0 & 5.5 & 11.0 & 12.0 & 13.0 & 14.0 \\
\hline$\alpha$ & $26.2 \%$ & $23.7 \%$ & $9.9 \%$ & $7.9 \%$ & $7.1 \%$ & $5.9 \%$ \\
ESJD & $3.26 \cdot 10^{-2}$ & $3.57 \cdot 10^{-2}$ & $5.69 \cdot 10^{-2}$ & $5.66 \cdot 10^{-2}$ & $5.89 \cdot 10^{-2}$ & $5.69 \cdot 10^{-2}$ \\
\hline
\end{tabular}

Even with these numerical imperfections, the results still echo what the theory predicts. Set the variance of the marginal proposal to be $\ell^{2} / n^{2}$ for different values of $\ell$. At $\ell=5.5$ the average acceptance rate is $23.7 \%$, with ESJD of $3.57 \cdot 10^{-2}$, while at $\ell=13.0$ the average acceptance rate is $7.1 \%$ with ESJD of $5.89 \cdot 10^{-3}$, which appears to be near optimal. Some other average acceptance rates and ESJD are reported in Table 1 (all numbers are based on a single RWM run of length $10^{5}$ ). The top left image of Figure 2 depicts the marginal target density. The top right compares the autocorrelation of the first coordinate of RWM algorithms for proposal variance tuned on the one hand to accept around $23 \%$ of the proposals $(\ell=5.5$, dashed line) and on the other hand to accept $7 \%$ of the proposals ( $\ell=13$, solid line) and to attain near optimal ESJD value. The bottom picture depicts $10^{4}$ steps of the first coordinate of the same RWM algorithms.

The observations of the acceptance rates, autocorrelation and ESJD are somewhat noisy, but it is clear that the ESJD values are the highest closer to the average acceptance rates of $7 \%$ rather than to $23 \%$. The autocorrelation also decays faster at $7 \%$ than at $23 \%$ of accepted proposals. Even the optimally tuned RWM exhibits slow mixing for the rough target.

An interesting feature we wish to note is the behaviour of the RWM path with near-optimal ESJD. It tends to occasionally get trapped in local modes for long periods of time, not accepting any proposal out of hundreds (see bottom image of Figure 2 around step 2000).
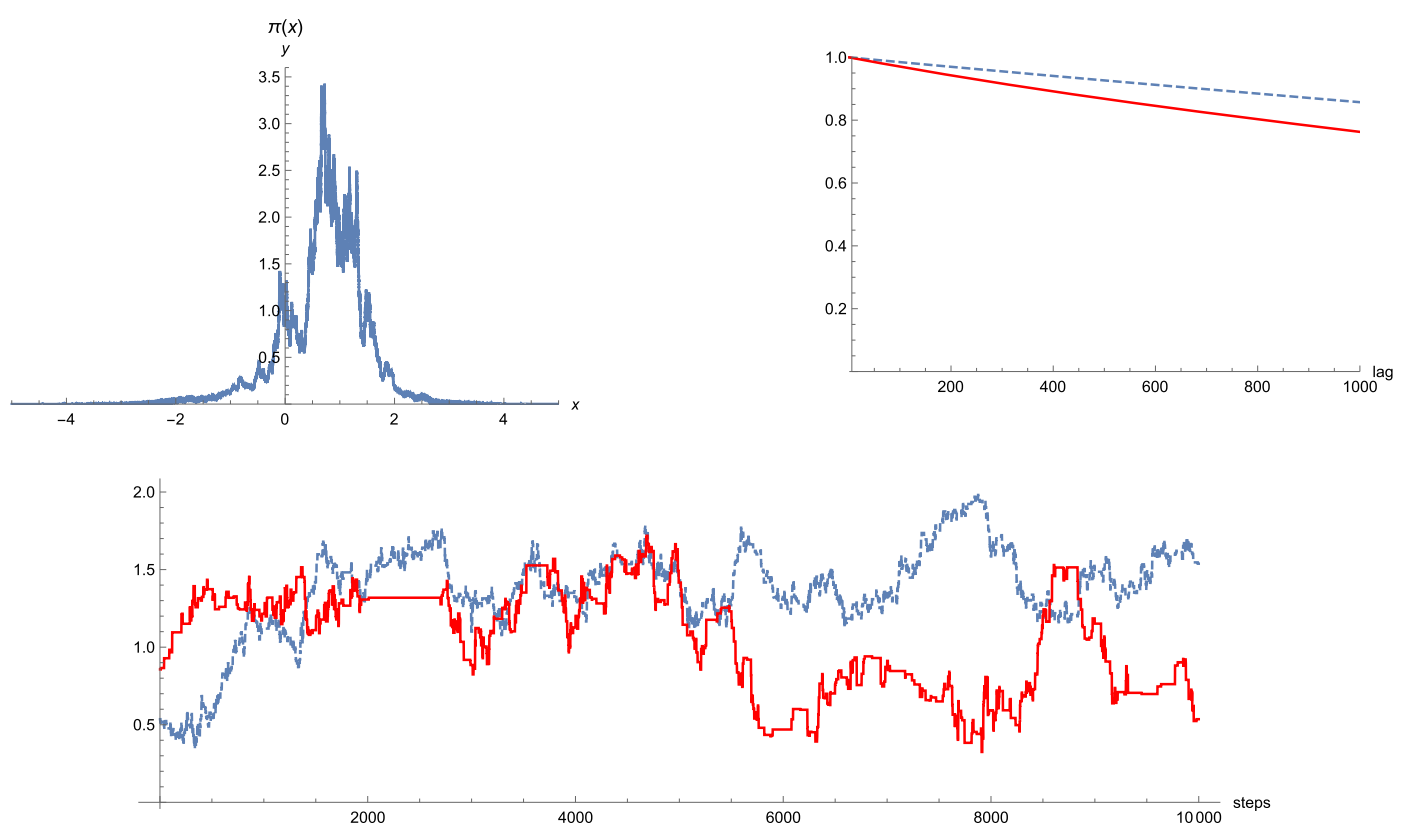

FIG. 2. Marginal target density $\pi$ on the top left. Autocorrelation (top right) and trace (bottom) plots of first coordinate of $R W M$ at $\ell=5.5$ tuned to accept $23 \%$ of proposals (dashed) and at $\ell=13.0$ with near optimal ESJD (solid). The near optimal ESJD scaling leads to behaviour of getting trapped in local modes. 
(b) Consideration of the theoretical counterexamples presented in Section 8.3(a) suggests that MCMC methods can get stuck in regions of high roughness in a manner similar to the way in which they can get stuck in local modes. Furthermore according to the MALA counterexample, these rough patches may only manifest at the level of the target derivatives, and hence may not be immediately detectable from the plot of the target, while still slowing down mixing. We would also expect problems in practice with application of RWM and MALA methods to finite dimensional targets falling in the regimes described in [29] and [30], but possessing regions of high local oscillations (at second order for MALA). In such cases one might expect to need to tune acceptance rate to a lower value than conventionally indicated.

Indeed, consider the following toy numerical example. Take an $n=100$ dimensional RWM chain with a product target defined by the requirement that the potential of the onedimensional marginal is

$$
\log (\pi(x))=-\frac{x^{2}}{2}+a \cos (b x)
$$

for constants $a=0.25, b=30$. Further take the proposal variance of marginal proposal to be equal to $\ell n^{-1}$. At $\ell=0.65$ the average acceptance rate is $23.3 \%$, with ESJD of $9.77 \cdot 10^{-4}$, while at $\ell=2.55$ the average acceptance rate is $7.7 \%$ with ESJD of $4.88 \cdot 10^{-3}$, which appears to be close to optimal. Some other average acceptance rates and ESJD are reported in Table 2 (all numbers are based on a single RWM run of length $10^{6}$ started in stationarity). Again the top left image of Figure 3 depicts the marginal target density. The top right compares the autocorrelation of the first coordinate of RWM algorithms for proposal variance tuned on the one hand to accept around $23 \%$ of the proposals $(\ell=0.65$, dashed line) and one the other hand to attain near optimal ESJD value $\left(\ell=2.55\right.$, solid line). The bottom picture depicts $10^{4}$ steps of the first coordinates of the same RWM algorithms.

Again the ESJD values and the autocorrelation plot suggest that RWM tuned to accept $7 \%$ of proposals outperforms the RWM tuned to accept $23 \%$ of proposals. The mixing is considerably faster than in the rough example 8.3(a) but is still slow.

We observe the same phenomenon as in Section 8.3(a). The solid line graph, corresponding to the optimally tuned proposal in terms of ESJD, has low acceptance rate and spends very long periods of time in particular states with high target density value.

Is this behaviour simply due to apparent multi-modality of the target? We do agree it is not unrelated, after all roughness and local oscillations are both in some sense extreme cases of local multi-modality. Note however, that the work of [29] assures us that for considerably larger $n$ we will see standard optimal scaling, despite the distance between neighbouring nodes relative to the proposal size not growing and modes becoming more pronounced due to multiplication of the marginal densities.

A natural question arises: can fixed deterministic marginal target densities of this kind be associated with an "appropriate" Hölder exponent? In this case we obtain the same acceptance rate as in Theorem 1 for $H \approx 0.5$, but it would be preferable to establish a link without having to optimise ESJD beforehand. If such a link can be established, can it be used together with

TABLE 2

Average acceptance rates $\alpha$ and ESJD for different values of $\ell$ for a smooth $R W M$ example

$\ell$

$\begin{array}{llllll}0.5 & 0.65 & 1.5 & 2 & 2.55 & 3\end{array}$

\begin{tabular}{lllllll}
\hline$\alpha$ & $29.3 \%$ & $23.3 \%$ & $14.7 \%$ & $11.1 \%$ & $7.7 \%$ & $5.2 \%$ \\
ESJD & $7.25 \cdot 10^{-4}$ & $9.77 \cdot 10^{-4}$ & $3.28 \cdot 10^{-3}$ & $4.37 \cdot 10^{-3}$ & $4.88 \cdot 10^{-3}$ & $4.58 \cdot 10^{-3}$ \\
\hline
\end{tabular}



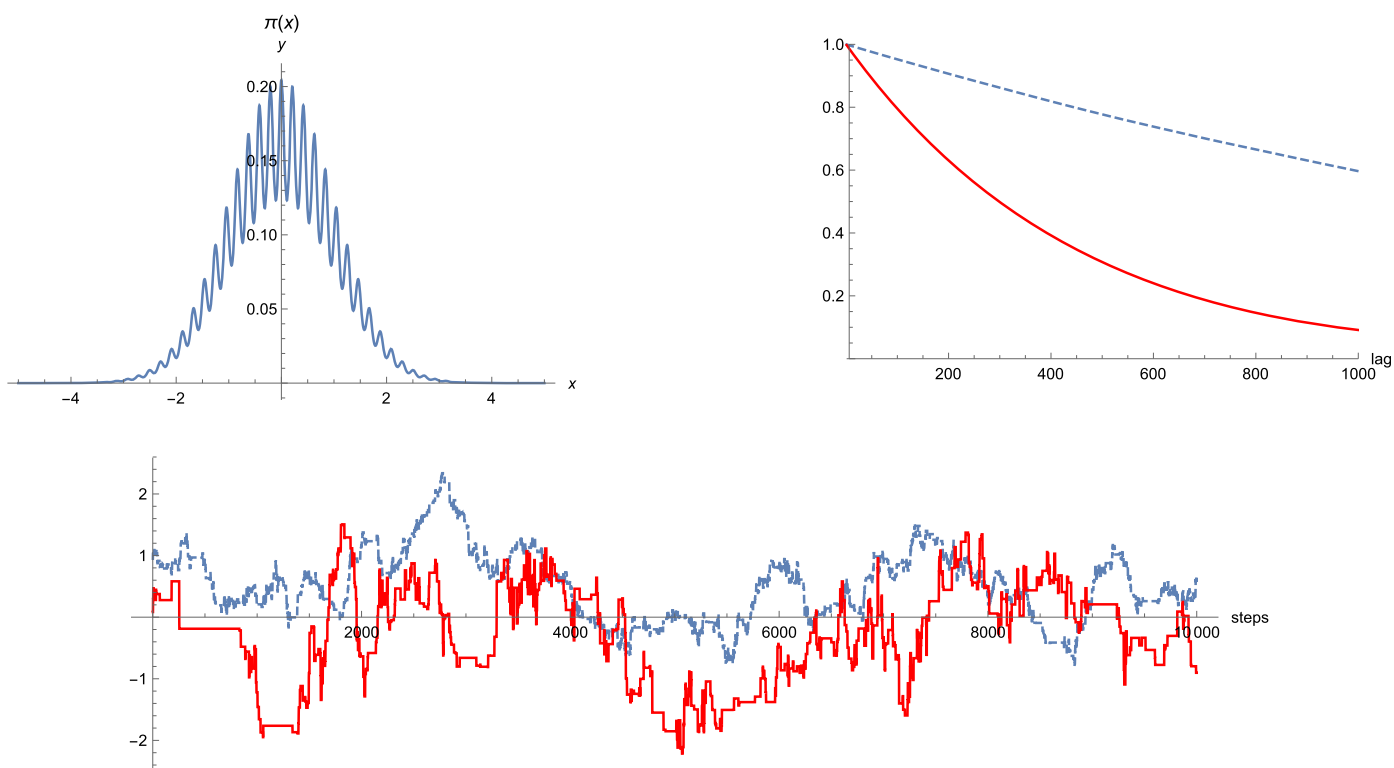

FIG. 3. Marginal target density $\pi$ on the top left. Autocorrelation (top right) and trace (bottom) plots of first coordinate of $R W M$ at $\ell=0.65$ tuned to accept $23 \%$ of proposals (dashed) and at $\ell=2.55$ with near optimal ESJD (solid). Again the near-optimal ESJD option leads to behaviour of getting trapped in local modes.

the insights of Theorem 1 to develop heuristics on how to tune the proposal variance for sampling from locally oscillatory or multi-modal targets?

Consider now a similar example for MALA instead of RWM. Take an $n=100$ dimensional MALA chain with the potential of the one-dimensional marginal equal to

$$
\log (\pi(x))=-\frac{x^{2}}{2}-\frac{a}{b^{2}} \cos (b x)
$$

for $a=0.9$ and $b=5$. This target is log concave and looks very much like the standard normal density and the oscillations only happen at the level of the second derivative of $\log (\pi)$ : we therefore do not present a figure. Take the proposal variance of the marginal proposal to be equal to $\ell n^{-\frac{1}{3}}$. Again, we can detect that the algorithm does not behave according to the theory [30] and has the best ESJD for lower acceptance rates. At $\ell=1.51$ the average acceptance rate is $57.4 \%$, with ESJD of 0.315 , while at $\ell=1.68$ the average acceptance rate is $47.5 \%$ with ESJD of 0.331 , which appears to be near optimal. Some other average acceptance rates and ESJD are reported in Table 3.

Results of Table 3 (each entry is again based on a single MALA run of length $10^{6}$ started at stationarity) are less precise then those of Table 2 as in the MALA case the ESJD do not vary so much over the range of average acceptance rates $45.2 \%-57.4 \%$ permitted by the conditions of Theorem 2. Moreover the numerical results suggest that, while there is detectable

TABLE 3

Average acceptance rates $\alpha$ and ESJD for different values of $\ell$ for the MALA example

\begin{tabular}{lrrrrrrrrr}
\hline & \multicolumn{10}{c}{$\ell$} \\
\cline { 2 - 9 } & 1.4 & 1.51 & 1.6 & 1.67 & 1.68 & 1.7 & 1.72 & 1.73 & 1.8 \\
\hline$\alpha$ & $62.9 \%$ & $57.4 \%$ & $52.4 \%$ & $48.1 \%$ & $47.5 \%$ & $46.3 \%$ & $45.2 \%$ & $44.5 \%$ & 40.0 \\
ESJD & 0.292 & 0.315 & 0.327 & 0.330 & 0.331 & 0.331 & 0.331 & 0.330 & 0.325 \\
\hline
\end{tabular}


deviation from classical results, nevertheless this does not have a significant impact on the performance of the method. Put differently, MALA tuned to accept anywhere between $45.2 \%$ and $57.4 \%$ of proposals works fine. Worrying about the roughness of the second derivative does not seem fruitful in practice.

The above considerations and these numerical examples suggest it would be valuable to conduct a thorough numerical study (using a variety of locally oscillating targets in different dimensions) to investigate this further. A referee suggests that it would be very interesting to compare (theoretically and numerically) the performance of an MCMC algorithm on a rough target with the performance on an associated truncated Karhunen-Loève expansion of the random part of the target. This might shed light on the way in which different levels of oscillation and roughness affect performance of MCMC algorithms.

(c) Sampling from targets with local oscillations is a matter of current scientific discussion, for instance, applications in disordered media and soft matter [3, 6, 10, 25, 27]. In light of our theoretical and numerical results it is sensible to argue that classic MCMC algorithms do not really work well for rough or oscillatory targets, and that one should seek appropriate modifications. Suggestions for such modifications also already exist [26]. Such modifications could usefully be assessed in terms of optimally tuned RWM and MALA algorithms providing benchmark MCMC methods.

It is also not impossible to imagine situations where one would want to sample rough targets, such as those in Section 8.3(a). This could happen naturally if rough targets are interpreted as noisy observations of a smooth target. This may be relevant for understanding pseudo-marginal Metropolis-Hastings algorithms [1, 2]. It is interesting to compare our results to the optimal scaling results for pseudo-marginal RWM obtained in Sherlock et al. [32], who in case of stationary Gaussian noise (Section 3.2) obtain the standard scaling of proposal variance $n^{-1}$ but the exact same optimal acceptance rate $7.001 \%$ as in our case for $H=\frac{1}{2}$. Again it seems possible that one could develop a common framework for studying noisy targets which would simultaneously explain both results.

Another more speculative usage of rough targets is when attempting to sample objects of fractal-like nature. Take Bayesian inference of ancestral trees as an example. There are a myriad ways in which an MCMC move on the space of trees can change the tree topology. Combined with complex likelihood structure arising when modelling mutations this seems capable of resulting in a setting that in the limit (of say growing number of tree leaves) approaches a rough target. In fact MCMC algorithms on trees do indeed suffer from very low acceptance rates when the proposal alters the tree topology, as reported for instance in Lakner et al. [20] and Höhna and Drummond [9]. Further investigation is needed to determine if this can be accounted for by some kind of effective roughness or local oscillations of the target.

\section{APPENDIX: AUXILIARY CALCULUS RESULTS}

This appendix establishes two simple lemmas concerning exact second-order Taylor expansions and a lemma establishing properties of a certain kind of set. All are used in the paper.

LEMMA A.1. Let $f \in \mathcal{C}^{2}(\mathbb{I})$ for an interval $\mathbb{I} \subset \mathbb{R}$. The following identity holds, provided that $x, x+\delta_{1}, x+\delta_{2}$, and $x+\delta_{1}+\delta_{2}$ all belong to $\mathbb{I}$ :

$$
f\left(x+\delta_{1}+\delta_{2}\right)-f\left(x+\delta_{1}\right)-f\left(x+\delta_{2}\right)+f(x)=\delta_{1} \delta_{2} \int_{0}^{1} \int_{0}^{1} f^{\prime \prime}\left(x+u \delta_{1}+v \delta_{2}\right) \mathrm{d} u \mathrm{~d} v .
$$

PROOF. The fundamental theorem of calculus implies that $F(y)-F(x)=(y-$ x) $\int_{0}^{1} F^{\prime}(x+u(y-x)) \mathrm{d} u$ holds for every $F \in \mathcal{C}^{1}(\mathbb{R})$ and all real $x, y$. This can be employed 
once for $F_{1}(x)=f\left(x+\delta_{1}\right)-f(x)$ and $y=x+\delta_{2}$, and then again for $F_{u}(x)=f^{\prime}\left(x+u \delta_{2}\right)$ and $y=x+\delta_{1}$, to yield

$$
\begin{aligned}
& f\left(x+\delta_{1}+\delta_{2}\right)-f\left(x+\delta_{1}\right)-f\left(x+\delta_{2}\right)+f(x) \\
& \quad=F_{1}\left(x+\delta_{2}\right)-F_{1}(x)=\delta_{2} \int_{0}^{1} F_{1}^{\prime}\left(x+u \delta_{2}\right) \mathrm{d} u \\
& \quad=\delta_{2} \int_{0}^{1} f^{\prime}\left(x+\delta_{1}+u \delta_{2}\right)-f^{\prime}\left(x+u \delta_{2}\right) \mathrm{d} u=\delta_{2} \int_{0}^{1} F_{u}\left(x+\delta_{1}\right)-F_{u}(x) \mathrm{d} u \\
& \quad=\delta_{1} \delta_{2} \int_{0}^{1} \int_{0}^{1} F_{u}^{\prime}\left(x+v \delta_{1}\right) \mathrm{d} v \mathrm{~d} u \\
& \quad=\delta_{1} \delta_{2} \int_{0}^{1} \int_{0}^{1} f^{\prime \prime}\left(x+u \delta_{1}+v \delta_{2}\right) \mathrm{d} u \mathrm{~d} v .
\end{aligned}
$$

LEMMA A.2. Let $f \in \mathcal{C}^{2}(\mathbb{R})$. The following holds for all real $x, \delta$ :

$$
f(x+\delta)-f(x)-\frac{\delta}{2}\left(f^{\prime}(x)+f^{\prime}(x+\delta)\right)=\frac{\delta^{2}}{2} \int_{0}^{1}(1-2 t) f^{\prime \prime}(x+t \delta) \mathrm{d} t .
$$

PROOF. Consider exact second-order Taylor expansions of $f(x+\delta)$ around $x$ and of $f(x)$ around $x+\delta$ :

$$
\begin{aligned}
f(x+\delta) & =f(x)+\delta f^{\prime}(x)+\int_{x}^{x+\delta} f^{\prime \prime}(u)(x+\delta-u) \mathrm{d} u \\
f(x) & =f(x+\delta)-\delta f^{\prime}(x+\delta)+\int_{x+\delta}^{x} f^{\prime \prime}(v)(x-v) \mathrm{d} v .
\end{aligned}
$$

These yield two different expansions for $f(x+\delta)-f(x)$. Averaging, we obtain

$$
\begin{aligned}
& f(x+\delta)-f(x)-\frac{\delta}{2}\left(f^{\prime}(x)+f^{\prime}(x+\delta)\right) \\
& \quad=\frac{1}{2} \int_{x}^{x+\delta} f^{\prime \prime}(u)(x+\delta-u) \mathrm{d} u-\frac{1}{2} \int_{x+\delta}^{x} f^{\prime \prime}(v)(x-v) \mathrm{d} v \\
& =\frac{\delta^{2}}{2}\left(\int_{0}^{1}(1-t) f^{\prime \prime}(x+t \delta) \mathrm{d} t-\int_{0}^{1} t f^{\prime \prime}(x+t \delta) \mathrm{d} t\right)=\frac{\delta^{2}}{2} \int_{0}^{1}(1-2 t) f^{\prime \prime}(x+t \delta) \mathrm{d} t,
\end{aligned}
$$

respectively using changes of variables $t=(u-x) / \delta$ and $t=(v-x) / \delta$.

LEMMA A.3. Let $\left\{a_{n}\right\}_{n \in \mathbb{N}}$ be a strictly decreasing positive sequence and denote for each $n \in \mathbb{N}$ the set

$$
\mathcal{S}_{n}:=\left\{\left(x_{1}, z_{1}, x_{2}, z_{2}\right) \in \mathbb{R}^{4}:\left|x_{1}-x_{2}\right|>2 a_{n}\left(\left|z_{1}\right|+\left|z_{2}\right|\right)\right\} .
$$

Then the following two statements hold:

(i) $\frac{1}{4 \pi^{2}} \int_{\mathcal{S}_{n}^{c}} e^{-\frac{1}{2}\left(x_{1}^{2}+z_{1}^{2}+x_{2}^{2}+z_{2}^{2}\right)} \mathrm{d} x_{1} \mathrm{~d} x_{2} \mathrm{~d} z_{1} \mathrm{~d} z_{2} \leq \frac{4}{\pi^{3 / 2}} \cdot a_{n}$.

(ii) For all $\left(x_{1}, z_{1}, x_{2}, z_{2}\right) \in \mathcal{S}_{n}$ and $u, v \in[0,1]$,

$$
\left|u z_{1}-v z_{2}\right| a_{n}<\frac{1}{2}\left|x_{1}-x_{2}\right|<\left|x_{1}-x_{2}-\left(u z_{1}-v z_{2}\right) a_{n}\right| .
$$


PROOF. Property (i): Consider the orthonormal change of variables given by $y_{1}=$ $\frac{1}{\sqrt{2}}\left(x_{1}-x_{2}\right), y_{2}=\frac{1}{\sqrt{2}}\left(x_{1}+x_{2}\right)$. This yields the following bound using the simple-minded bound $\int_{-a}^{a} e^{-y_{1}^{2} / 2} \mathrm{~d} y_{1} \leq 2 a$ :

$$
\begin{aligned}
& \frac{1}{4 \pi^{2}} \int_{\mathcal{S}_{n}^{c}} e^{-\frac{1}{2}\left(x_{1}^{2}+z_{1}^{2}+x_{2}^{2}+z_{2}^{2}\right)} \mathrm{d} x_{1} \mathrm{~d} x_{2} \mathrm{~d} z_{1} \mathrm{~d} z_{2} \\
& \quad=\frac{1}{2 \pi} \int_{\mathbb{R}^{2}} e^{-\frac{1}{2}\left(z_{1}^{2}+z_{2}^{2}\right)}\left(\frac{1}{2 \pi} \int_{\left|y_{1}\right| \leq \sqrt{2} a_{n}\left(\left|z_{1}\right|+\left|z_{2}\right|\right)} e^{-\frac{1}{2}\left(y_{1}^{2}+y_{2}^{2}\right)} \mathrm{d} y_{1} \mathrm{~d} y_{2}\right) \mathrm{d} z_{1} \mathrm{~d} z_{2} \\
& \quad \leq \frac{\sqrt{2} a_{n}}{\pi} \cdot \frac{1}{2 \pi} \iint_{\mathbb{R}^{2}}\left(\left|z_{1}\right|+\left|z_{2}\right|\right) e^{-\frac{1}{2}\left(z_{1}^{2}+z_{2}^{2}\right)} \mathrm{d} z_{1} \mathrm{~d} z_{2} \\
& \quad=\frac{2 \sqrt{2} a_{n}}{\pi} \cdot \frac{1}{2 \pi} \iint_{\mathbb{R}^{2}}\left|z_{1}\right| e^{-\frac{1}{2}\left(z_{1}^{2}+z_{2}^{2}\right)} \mathrm{d} z_{1} \mathrm{~d} z_{2}=\frac{4}{\pi^{3 / 2}} \cdot a_{n} .
\end{aligned}
$$

Property (ii): Working with the definition of $\mathcal{S}_{n}$, we deduce

$$
\left|u z_{1}-v z_{2}\right| a_{n} \leq\left(\left|z_{1}\right|+\left|z_{2}\right|\right) a_{n}<\frac{1}{2}\left|x_{1}-x_{2}\right| \text {. }
$$

On the other hand,

$$
\begin{aligned}
\frac{1}{2}\left|x_{1}-x_{2}\right| & =\left|x_{1}-x_{2}\right|-\frac{1}{2}\left|x_{1}-x_{2}\right|<\left|x_{1}-x_{2}\right|-\left|u z_{1}-v z_{2}\right| a_{n} \\
& \leq\left|x_{1}-x_{2}-\left(u z_{1}-v z_{2}\right) a_{n}\right| .
\end{aligned}
$$

Acknowledgements. This work was funded by the UK EPSRC under grant EP/R022100. The second author acknowledges the support of the Alan Turing Institute under EPSRC grant EP/N510129.

We thank Jere Koskela, Anastasia Papavasiliou and Giacomo Zanella for useful debate about material presented in the Discussion.

\section{REFERENCES}

[1] Andrieu, C. and Roberts, G. O. (2009). The pseudo-marginal approach for efficient Monte Carlo computations. Ann. Statist. 37 697-725. MR2502648 https://doi.org/10.1214/07-AOS574

[2] Beaumont, M. A. (2003). Estimation of population growth or decline in genetically monitored populations. Genetics 164 1139-1160.

[3] Ben Arous, G. and Owhadi, H. (2003). Multiscale homogenization with bounded ratios and anomalous slow diffusion. Comm. Pure Appl. Math. 56 80-113. MR1929443 https://doi.org/10.1002/cpa.10053

[4] Christensen, O. F., Roberts, G. O. and Rosenthal, J. S. (2005). Scaling limits for the transient phase of local Metropolis-Hastings algorithms. J. R. Stat. Soc. Ser. B. Stat. Methodol. 67 253-268. MR2137324 https://doi.org/10.1111/j.1467-9868.2005.00500.x

[5] Diaconis, P. (2013). Some things we've learned (about Markov chain Monte Carlo). Bernoulli 191294 1305. MR3102552 https://doi.org/10.3150/12-BEJSP09

[6] Duncan, A. B., Kalliadasis, S., Pavliotis, G. A. and Pradas, M. (2016). Noise-induced transitions in rugged energy landscapes. Phys. Rev. E 94 032107. https://doi.org/10.1103/PhysRevE.94.032107

[7] Durmus, A., Le CorfF, S., Moulines, E. and Roberts, G. O. (2017). Optimal scaling of the random walk Metropolis algorithm under $L^{p}$ mean differentiability. J. Appl. Probab. 54 1233-1260. MR3731293 https://doi.org/10.1017/jpr.2017.61

[8] Gelfand, S. B. and MitTer, S. K. (1991). Weak convergence of Markov chain sampling methods and annealing algorithms to diffusions. J. Optim. Theory Appl. 68 483-498. MR1097314 https://doi.org/10. 1007/BF00940066

[9] Höhna, S. and Drummond, A. J. (2012). Guided tree topology proposals for Bayesian phylogenetic inference. Syst. Biol. 61 1-11. https://doi.org/10.1093/sysbio/syr074

[10] HU, M. and BAO, J.-D. (2018). Diffusion crossing over a barrier in a random rough metastable potential. Phys. Rev. E 97062143. 
[11] ISSERLIS, L. (1918). On a formula for the product-moment coefficient of any order of a normal frequency distribution in any number of variables. Biometrika 12 134-139.

[12] Janson, S. (1997). Gaussian Hilbert Spaces. Cambridge Tracts in Mathematics 129. Cambridge Univ. Press, Cambridge. MR1474726 https://doi.org/10.1017/CBO9780511526169

[13] Jourdain, B., Lelièvre, T. and Miasojedow, B. (2014). Optimal scaling for the transient phase of Metropolis Hastings algorithms: The longtime behavior. Bernoulli 20 1930-1978. MR3263094 https://doi.org/10.3150/13-BEJ546

[14] Jourdain, B., Lelièvre, T. and Miasojedow, B. (2015). Optimal scaling for the transient phase of the random walk Metropolis algorithm: The mean-field limit. Ann. Appl. Probab. 25 2263-2300. MR3349007 https://doi.org/10.1214/14-AAP1048

[15] Kallenberg, O. (2010). Foundations of Modern Probability, 2nd ed. Springer, Berlin.

[16] Kendall, W. S. (1980). Contours of Brownian processes with several-dimensional times. Z. Wahrsch. Verw. Gebiete 52 267-276. MR0576887 https://doi.org/10.1007/BF00538891

[17] Kendall, W. S. (1982). Contours and Baire category. Bull. Lond. Math. Soc. 14 30-32. MR0642419 https://doi.org/10.1112/blms/14.1.30

[18] Kuntz, J., Ottobre, M. and Stuart, A. M. (2018). Non-stationary phase of the MALA algorithm. Stoch. Partial Differ. Equ. Anal. Comput. 6 446-499. MR3844656 https://doi.org/10.1007/ s40072-018-0113-1

[19] Kuntz, J., Ottobre, M. and Stuart, A. M. (2019). Diffusion limit for the random walk Metropolis algorithm out of stationarity. Ann. Inst. Henri Poincaré Probab. Stat. 55 1599-1648. MR4010946 https://doi.org/10.1214/18-aihp929

[20] Lakner, C., van der Mark, P., Huelsenbeck, J. P., Larget, B. and Ronquist, F. (2008). Efficiency of Markov chain Monte Carlo tree proposals in Bayesian phylogenetics. Syst. Biol. 57 86-103. https://doi.org/10.1080/10635150801886156

[21] Mandelbrot, B. B. and Van Ness, J. W. (1968). Fractional Brownian motions, fractional noises and applications. SIAM Rev. 10 422-437. MR0242239 https://doi.org/10.1137/1010093

[22] Mijatović, A. and Vogrinc, J. (2019). Asymptotic variance for random walk Metropolis chains in high dimensions: Logarithmic growth via the Poisson equation. Adv. in Appl. Probab. 51 994-1026. MR4032170 https://doi.org/10.1017/apr.2019.40

[23] Neal, P., Roberts, G. and Yuen, W. K. (2012). Optimal scaling of random walk Metropolis algorithms with discontinuous target densities. Ann. Appl. Probab. 22 1880-1927. MR3025684 https://doi.org/10. 1214/11-AAP817

[24] Nualart, D. (2006). The Malliavin Calculus and Related Topics, 2nd ed. Probability and Its Applications (New York). Springer, Berlin. MR2200233

[25] Owhadi, H. (2003). Anomalous slow diffusion from perpetual homogenization. Ann. Probab. 31 19351969. MR2016606 https://doi.org/10.1214/aop/1068646372

[26] Plecháč, P. and Simpson, G. (2019). Sampling from rough energy landscapes. Preprint. Available at arXiv:1903.09998.

[27] Pollak, E., Auerbach, A. and Talkner, P. (2008). Observations on rate theory for rugged energy landscapes. Biophys. J. 95 4258-4265.

[28] RevuZ, D. and Yor, M. (1991). Continuous Martingales and Brownian Motion. Grundlehren der Mathematischen Wissenschaften [Fundamental Principles of Mathematical Sciences] 293. Springer, Berlin. MR1083357 https://doi.org/10.1007/978-3-662-21726-9

[29] Roberts, G. O., Gelman, A. and Gilks, W. R. (1997). Weak convergence and optimal scaling of random walk Metropolis algorithms. Ann. Appl. Probab. 7 110-120. MR1428751 https://doi.org/10. 1214/aoap/1034625254

[30] Roberts, G. O. and Rosenthal, J. S. (1998). Optimal scaling of discrete approximations to Langevin diffusions. J. R. Stat. Soc. Ser. B. Stat. Methodol. 60 255-268. MR1625691 https://doi.org/10.1111/ $1467-9868.00123$

[31] Roberts, G. O. and Rosenthal, J. S. (2001). Optimal scaling for various Metropolis-Hastings algorithms. Statist. Sci. 16 351-367. MR1888450 https://doi.org/10.1214/ss/1015346320

[32] Sherlock, C., Thiery, A. H., Roberts, G. O. and Rosenthal, J. S. (2015). On the efficiency of pseudo-marginal random walk Metropolis algorithms. Ann. Statist. 43 238-275. MR3285606 https://doi.org/10.1214/14-AOS1278

[33] Tierney, L. (1998). A note on Metropolis-Hastings kernels for general state spaces. Ann. Appl. Probab. 8 1-9. MR1620401 https://doi.org/10.1214/aoap/1027961031

[34] Yang, J., Roberts, G. O. and Rosenthal, J. S. (2019). Optimal scaling of metropolis algorithms on general target distributions. Available at arXiv:1904.12157.

[35] Zanella, G., BÉdARD, M. and Kendall, W. S. (2017). A Dirichlet form approach to MCMC optimal scaling. Stochastic Process. Appl. 127 4053-4082. MR3718106 https://doi.org/10.1016/j.spa.2017.03. 021 